\title{
Fiducial $q_{T}$ resummation of color-singlet processes at $\mathrm{N}^{3} \mathrm{LL}+\mathrm{NNLO}$
}

\author{
Thomas Becher ${ }^{a}$ and Tobias Neumann ${ }^{b, c}$ \\ ${ }^{a}$ Albert Einstein Center for Fundamental Physics, Institut für Theoretische Physik, \\ Universität Bern, Sidlerstrasse 5, CH-3012 Bern, Switzerland \\ ${ }^{b}$ Fermilab, \\ PO Box 500, Batavia, Illinois 60510, U.S.A. \\ ${ }^{c}$ Department of Physics, Illinois Institute of Technology, \\ Chicago, Illinois 60616, U.S.A. \\ E-mail: becher@itp.unibe.ch, tneumann@fnal.gov
}

ABSTRACT: We present a framework for $q_{T}$ resummation at $\mathrm{N}^{3} \mathrm{LL}+\mathrm{NNLO}$ accuracy for arbitrary color-singlet processes based on a factorization theorem in SCET. Our implementation CuTe-MCFM is fully differential in the Born kinematics and matches to large- $q_{T}$ fixed-order predictions at relative order $\alpha_{s}^{2}$. It provides an efficient way to estimate uncertainties from fixed-order truncation, resummation, and parton distribution functions. In addition to $W^{ \pm}, Z$ and $H$ production, also the diboson processes $\gamma \gamma, Z \gamma, Z H$ and $W^{ \pm} H$ are available, including decays. We discuss and exemplify the framework with several direct comparisons to experimental measurements as well as inclusive benchmark results. In particular, we present novel results for $\gamma \gamma$ and $Z \gamma$ at $\mathrm{N}^{3} \mathrm{LL}+\mathrm{NNLO}$ and discuss in detail the power corrections induced by photon isolation requirements.

KEYwords: NLO Computations, QCD Phenomenology

ArXiv ePrint: 2009.11437 


\section{Contents}

1 Introduction 1

2 Resummation framework and implementation 5

3 Results $\quad 16$

$\begin{array}{lll}3.1 & \text { Benchmark calculations and comparison with } \mathrm{CuTe} & 17\end{array}$

3.1.1 Inclusive Higgs production 18

3.1.2 Inclusive $Z$ production 20

$3.2 \quad$ Fiducial $Z$ production 21

3.2.1 ATLAS measurements at $8 \mathrm{TeV} \quad 22$

3.2.2 CMS measurements at $13 \mathrm{TeV} \quad 25$

3.3 Fiducial $W$ production as measured by CMS at $8 \mathrm{TeV}$

3.4 Fiducial $H \rightarrow \gamma \gamma$ benchmark 28

$\begin{array}{lll}3.5 & \text { Fiducial } \gamma \gamma \text { production } & 28\end{array}$

$\begin{array}{lll}3.5 .1 & \text { ATLAS measurements at } 7 \mathrm{TeV} & 30\end{array}$

$\begin{array}{ll}\text { 3.5.2 ATLAS measurements at } 8 \mathrm{TeV} & 35\end{array}$

3.6 Fiducial $Z \gamma$ production 37

3.6.1 ATLAS measurements at $13 \mathrm{TeV} \quad 40$

4 Conclusions $\quad 44$

$\begin{array}{ll}\text { A PDF uncertainties } & 46\end{array}$

\section{Introduction}

While hadron colliders were traditionally considered discovery machines, one cannot deny the success of the Large Hadron Collider (LHC) experiments in Standard Model (SM) precision physics. Already today, differential measurements at sub-percent level precision are available, a prime example being the transverse-momentum $\left(q_{T}\right)$ spectrum of the $Z$ boson. A related precision measurement at the LHC is the extraction of the $W$-boson mass by the ATLAS collaboration [1], heavily relying on a precise understanding of the charged lepton transverse-momentum distribution through a template fit.

Transverse-momentum distributions and the experimentally easier to measure, but closely associated, $\phi^{*}[2]$ distributions in electroweak boson production are key observables for SM precision tests. For example, the precise measurements and predictions of $Z$-boson transverse-momentum spectra allow for significant constraints on PDFs [3] and might help to resolve tensions in existing PDF fits. The large data sets of the LHC also allow for increasingly precise diboson production measurements [4-12], which are key to test the gauge 
structure of the SM, as was pointed out a long time ago $[13,14]$. Recent theoretical studies of such processes include refs. [15-18]. To increase sensitivity to Beyond the Standard Model (BSM) effects it is important to veto QCD radiation. The transverse momentum can be used as a kinematic variable to veto jets, see e.g. ref. [16], and is therefore relevant also in the search for BSM physics. It is therefore essential for the physics program at the LHC that theoretical predictions and associated uncertainties for these processes are under good control.

Predictions in fixed-order perturbation theory at hadron colliders start with a collinear factorization theorem involving parton distribution functions and a hard scattering cross section at a scale $Q$, corresponding to the invariant mass of the final-state electroweak bosons. However, when considering the kinematical distributions of transverse momenta at small values, fixed-order corrections are enhanced by large Sudakov logarithms of scale ratios $Q^{2} / q_{T}^{2}$. To obtain meaningful results, the fixed-order predictions need to be improved with an all-order resummation of such logarithms. In this paper we address this issue by combining the fixed-order color-singlet NNLO processes in MCFM [19-22] with the SCETbased $q_{T}$ resummation at $\mathrm{N}^{3} \mathrm{LL}$ introduced in refs. [23-26]. The resulting code CuTe-MCFM will be made publicly available shortly at https://mcfm.fnal.gov.

Transverse-momentum resummation in SCET. The enhanced logarithms for small transverse momenta are universal and originate from soft and collinear radiation. An allorder exponentiation theorem for the $q_{T}$ distribution was first obtained in ref. [27] and is now known as the Collins-Soper-Sterman (CSS) formula.

Two sources of enhanced terms exist. First, logarithms arising due to different scales associated with the hard process and the soft/collinear radiation, and, secondly, logarithms generated by the rapidity difference of small- $q_{T}$ emissions from partons flying along the beams to the left and right. In SCET $[28-30]^{1}$ the first kind of logarithms are resummed by solving the renormalization group equations (RGEs) of the derived factorization theorem in the limit of small $q_{T}$. This was first considered in refs. [34-36] without accounting for the rapidity logarithms. Later, both sources of logarithms have been taken into account for $q \bar{q}$-initiated processes [23, 24] and for $g g$-initiated processes [25, 37], and the equivalence to the CSS formula was established. Instead of a direct exponentiation [23], the rapidity logarithms can also be resummed by solving rapidity RGEs [37, 38].

Resummation codes. A number of computer codes for transverse-momentum resummation of color-singlet processes have been developed, some of which have been made publicly available. They differ by the achieved logarithmic precision, the possibility of fiducial cuts on the final state colorless particles, and in subleading terms through the use of different resummation and matching formalisms, in particular whether the computations are performed in momentum or impact parameter space.

Fiducial resummation in Drell-Yan production is available through DYRes [39, 40], its new implementation DYTurbo [41] and ReSolve [42] at $\mathrm{N}^{2} \mathrm{LL}^{\prime}$, and at $\mathrm{N}^{3} \mathrm{LL}$ without the pos-

\footnotetext{
${ }^{1}$ See $[31-33]$ for reviews.
} 
sibility for fiducial cuts in CuTe $[24,25] .{ }^{2}$ In addition, there are codes such as arTeMiDe [43] and NangaParbat [44], with a special focus on non-perturbative transverse-momentum dependent (TMD) physics. In a recent paper [45] $\mathrm{N}^{3} \mathrm{LL}$ fiducial results have been presented based on the private code SCETlib. Fiducial resummation for Higgs production is available through HRes $[46,47]$ at $\mathrm{N}^{2} \mathrm{LL}^{\prime}$. Codes for resummation in $W^{ \pm}, Z, H, \gamma \gamma$ and $Z Z$ from various authors and at different accuracies are available under the name Resbos/Resbos2 [48-50]. For $W W$ and $Z Z$ production results at $\mathrm{N}^{2} \mathrm{LL}$ have been presented in ref. [51].

The above results are based on analytic computations of the ingredients of the factorization theorem for the process at small transverse momentum. An alternative numerical resummation technique was developed in refs. [52-54]. In this formalism, the higher emissions are computed with Monte-Carlo methods. This numerical approach was generalised to transverse-momentum resummation in ref. [55] and extended to $\mathrm{N}^{3} \mathrm{LL}$ in ref. [56]. The resulting resummation framework (RadISH) has been interfaced to fixed-order codes for different $2 \rightarrow 1$ and $2 \rightarrow 2$ color-singlet processes [57], which provide matching to order $\alpha_{s}^{2}$ at large $q_{T}[58,59]$ (MATRIX+RadISH), and even to order $\alpha_{s}^{3}$ for Higgs [56] and $W$ and $Z$ production [60-62] (RadISH+NNLOJet).

It is of course also common, especially in the experimental collaborations, to rely on parton showers to dress fixed-order predictions with logarithmically enhanced terms [63]. While these showers typically give a good description of experimentally measured spectra, they do not systematically include higher logarithmic terms and need to be benchmarked against analytical resummation results such as the ones in this study.

The modern approach of matching and merging often achieves impressive results in predicting shapes of distributions, but the low logarithmic accuracy can be problematic. Cross sections differential in transverse momentum typically peak around small values, so the bulk of the cross section comes from the region that needs an all-order resummation. Therefore, it is clear that the fixed-order and logarithmic precision in this bulk region should be as high as possible. General purpose parton shower codes typically only reach fixed NLO accuracy and leading logarithmic accuracy in the region of small $q_{T}$. For normalized distributions this limitation can amplify and even invalidate the formal NLO perturbative accuracy achieved in the fixed-order tail regions [64]. It is therefore important to use - or at least compare with - predictions that have known parametric accuracy and allow for systematic estimates of uncertainties.

Scheme choices. The product form of the $q_{T}$-factorization formula arises in transverse position space, also known as impact parameter space. Following CSS, it is therefore common to perform the resummation in impact parameter space and then compute the Fourier integral to obtain the transverse-momentum spectrum. A disadvantage of this procedure is that one ends up with running couplings that are functions of the impact parameter $b$, which is integrated from zero to infinity in the Fourier integral. This makes it necessary to choose a prescription to avoid Landau pole singularities. In the effective theory approach [23, 24] based on RG evolution, which we adopt in our work, one instead first carries out the Fourier

\footnotetext{
${ }^{2}$ The papers $[24,25]$ achieved $\mathrm{N}^{2} \mathrm{LL}$, but the accuracy was extended in version 2 of the CuTe code, see https://cute.hepforge.org.
} 
integral and then sets the boundary conditions of the evolution directly in $q_{T}$ space. The rapidity logarithms, on the other hand, which do not involve a running of the coupling, are exponentiated in position space in the formalism of refs. [23, 24]. A method to resum all logarithms in $q_{T}$ space has been developed in ref. [65], but is challenging to implement. In any case, performing the resummation in different spaces simply amounts to choosing different boundary conditions, which induce different subleading terms and different power corrections.

A second source of subleading differences, on top of the choice of resummation space, is the matching to the fixed-order predictions [66]. A robust estimation of perturbative uncertainties therefore benefits from fully matched results in different matching schemes and resummation formalisms. To some extend these effects can be estimated within one framework, of course. For example, in our study we use a transition function to match our resummed results to fixed-order predictions. Varying this function provides a flexible way to estimate matching uncertainties. One could furthermore deliberately choose to include different subleading terms in the resummation. Overall our $\mathrm{N}^{3} \mathrm{LL}+\mathrm{NNLO}$ resummation framework allows for the estimation of QCD uncertainties through variation of the renormalization, resummation and factorization scales ("scale uncertainties"), PDF $+\alpha_{s}$ uncertainties, and matching uncertainties by varying the transition function. The combination of these should capture the bulk of uncertainties associated with a perturbative QCD prediction.

Overview of the paper. In this work we present a SCET-derived transverse-momentum resummation framework and publicly available implementation CuTe-MCFM to calculate fully matched predictions with fiducial cuts at $\mathrm{N}^{3} \mathrm{LL}+\mathrm{NNLO}\left(\alpha_{s}^{2}\right.$ relative to the Born). The name CuTe-MCFM was chosen to emphasize that the implementation is based on refs. [23, $24]$ as the earlier public code CuTe. However, while we performed various cross checks against this earlier code, CuTe-MCFM is a new and completely independent implementation of the underlying equations. The code follows the same philosophy as ref. [26], in that it uses an existing fixed-order code to compute the process-dependent parts of the resummation formula. Interfacing to MCFM provides an efficient way of studying different processes and allows us to take into account the decays of the electroweak bosons as well as cuts on the decay products.

While implemented in MCFM, the code written for this study is not closely tied to MCFM, so that it could easily be reused or integrated in other situations, for example as a stand-alone extension of the interface to event files used in ref. [26], that is currently limited to $\mathrm{N}^{2} \mathrm{LL}$ and quark-antiquark initiated processes. The only essential input ingredients are the Born matrix element, the hard function at relative order $\alpha_{s}$ or $\alpha_{s}^{2}$, and numerically stable fixed-order predictions at $q_{T}>0$ for matching.

Relative to the Born-level boson production process, our framework achieves $\alpha_{s}^{2}$ accuracy both at small and large $q_{T}$ through a consistent power counting of $\alpha_{s}$ and large logarithms. We demonstrate our implementation with fully matched kinematical distributions in $q_{T}, \phi^{*}$, and with distributions in the azimuthal angle difference $\Delta \phi$ between bosons. We estimate scale uncertainties, PDF uncertainties and matching uncertainties, 
and also address the impact of fiducial cuts on the size of subleading power corrections in the $q_{T}$ factorization. We include a detailed discussed of power corrections in processes involving photons, in which they are enhanced through the required photon isolation cuts.

In section 2 we describe our framework and setup in detail. We discuss the factorization theorem, the resummation of large logarithms through RG evolution and exponentiation of rapidity logarithms, the estimation of scale uncertainties and PDF uncertainties, matching to fixed-order predictions and differences to the code CuTe. We discuss in detail subleading power corrections from fiducial cuts and photon isolation. In addition, we provide details about the technical implementation, for example the ability to pre-generate beamfunction grids.

In section 3 we compare with the CuTe code for $Z$ and $H$ production. We then show results for various processes with fiducial cuts and in comparison with experimental measurements. For $Z$ production we compare with measurements at $13 \mathrm{TeV}$ and $8 \mathrm{TeV}$. For $Z \gamma$ production we compare with recent experimental data at $13 \mathrm{TeV}$ and show novel results that have previously only been considered in fixed-order perturbation theory. For diphoton production we compare against data at $7 \mathrm{TeV}$ and recent data at $8 \mathrm{TeV}$ and improve upon previous predictions at $\mathrm{N}^{2} \mathrm{LL}$. We show results for Higgs production, both inclusively as part of our comparison with $\mathrm{CuTe}$, and in the $H \rightarrow \gamma \gamma$ decay mode with fiducial cuts. We do not compare against the measured $q_{T}$ distribution for Higgs production that still has large uncertainties. The comparison would require a careful analysis of multiple production channels and top-quark mass effects, among other things, which go beyond the scope of our study. We furthermore compare with one of the few direct $W$ boson transverse momentum measurements. Resummation for the remaining processes $Z H$ and $W^{ \pm} H$ is prepared in our code and ready for use. We conclude in section 4 and present an outlook for future studies based on this work.

\section{Resummation framework and implementation}

Factorization formula. The $q_{T}$ resummation underlying our framework CuTe-MCFM has been derived in SCET in refs. [23-25], where large logarithms of argument $q_{T} / Q$ are resummed through RG evolution of hard function and beam functions, and rapidity logarithms are directly exponentiated through the collinear-anomaly formalism.

The production of multiple weak bosons in this formalism has been detailed in ref. [26]. As in this work, we consider the production of $N$ weak bosons with momenta $\{\underline{q}\}=$ $\left\{q_{1}, q_{2}, \ldots, q_{N}\right\}$. The total boson momentum is denoted by $q^{\mu}=q_{1}^{\mu}+\cdots+q_{N}^{\mu}$ and the resummation formalism is valid in the region where the transverse momentum $q_{T}=\sqrt{-q_{\perp}^{2}}$ is much smaller than the invariant mass $Q^{2}=q^{2}$ of the electroweak final state.

The cross section is a sum of contributions from individual partonic channels $i, j \in$ $q, \bar{q}, g$. Up to terms suppressed by powers of $q_{T}$, these channels factorize as

$$
\begin{aligned}
\mathrm{d} \sigma_{i j}\left(p_{1}, p_{2},\{\underline{q}\}\right)= & \int_{0}^{1} \mathrm{~d} \xi_{1} \int_{0}^{1} \mathrm{~d} \xi_{2} \mathrm{~d} \sigma_{i j}^{0}\left(\xi_{1} p_{1}, \xi_{2} p_{2},\{\underline{q}\}\right) \mathcal{H}_{i j}\left(\xi_{1} p_{1}, \xi_{2} p_{2},\{\underline{q}\}, \mu\right) \\
& \cdot \frac{1}{4 \pi} \int \mathrm{d}^{2} x_{\perp} e^{-i q_{\perp} x_{\perp}}\left(\frac{x_{T}^{2} Q^{2}}{b_{0}^{2}}\right)^{-F_{i j}\left(x_{\perp}, \mu\right)} B_{i}\left(\xi_{1}, x_{\perp}, \mu\right) \cdot B_{j}\left(\xi_{2}, x_{\perp}, \mu\right),
\end{aligned}
$$


where $p_{1}$ and $p_{2}$ are the incoming hadron momenta. The cross section $\mathrm{d} \sigma_{i j}$ is fully differential in the electroweak momenta $\{\underline{q}\}$.

The beam functions $B_{i}$ and $B_{j}$ encode the soft and collinear emissions at low transverse momentum (or more precisely large transverse separation $x_{\perp}$ ) and the indices $i$ and $j$ and the momentum fractions $\xi_{1}$ and $\xi_{2}$ refer to the partons which enter the hard process after these emissions. The hard Born-level process has the differential cross section $\mathrm{d} \sigma_{i j}^{0}$ and the hard-function as $\mathcal{H}_{i j}$ collects the associated virtual corrections. The collinear anomaly leads to the $Q^{2}$-dependent factor within the Fourier-integral over the transverse position $x_{\perp}$. The perturbatively calculable anomaly exponent $F_{i j}$ is also referred to as the rapidity anomalous dimension in the framework of ref. [37]. In case of gluon-gluon initiated processes $(i=j=g)$, a second product of beam functions is added as required [25, 67]. Lastly, we have defined $b_{0}=2 e^{-\gamma_{E}}$, where $\gamma_{E}$ is the Euler-Mascheroni constant, and $x_{T}^{2}=-x_{\perp}^{2}$.

The hard function and the Born cross section are the only process-dependent ingredients in formula (2.1). Since the hard function corresponds to the $\overline{M S}-$ renormalized loop corrections to the Born amplitude and the implementations of NNLO corrections in MCFM are based upon a SCET-derived factorization for jettiness $\tau$ [68], the $\overline{\text { MS}}$-renormalized hard functions are readily available. Furthermore, the processes associated with $\tau>0$ correspond to those with $q_{T}>0$ needed for the fixed-order matching, and are already well-tested and numerically stable in the singular limits.

The hard function involves logarithms of the ratio $\mu^{2} / Q^{2}$, which are minimized with a choice of $\mu=\mu_{h}^{2} \sim Q^{2}$, but inside the beam functions the natural choice is $\mu \sim q_{T}$. To avoid large logarithms of $q_{T}^{2} / Q^{2}$ one chooses $\mu_{h} \sim Q$ in the hard function and then evolves it down to the resummation scale $\mu \sim q_{T}$ using the RG. This evolution can be solved analytically to obtain a hard function evolution factor $U\left(Q^{2}, \mu_{h}, \mu\right)$ with cusp anomalous dimension and quark and gluon anomalous dimensions as essential ingredients, see ref. [69] for details. At $\mathrm{N}^{3} \mathrm{LL}$ we make use of the recent calculation of the four-loop cusp anomalous dimension [70-72].

The appearance of the power-like dependence on the hard scale $Q^{2}$ from a refactorization of regularized beam functions has been discussed extensively in refs. [23, 24], where the associated anomaly exponent $F_{i j}$ was first extracted to two-loop accuracy. For resummation at $\mathrm{N}^{3} \mathrm{LL}$ we use the three-loop result of refs. [73, 74].

Improvement at very small $\boldsymbol{q}_{T}$. It is natural to rewrite the anomaly as a function of the logarithm $L_{\perp}=\log \left(x_{T}^{2} \mu^{2} / b_{0}^{2}\right)$ and the quantity

$$
\eta_{i}=\frac{C_{i} \alpha_{s}(\mu)}{\pi} \log \frac{Q^{2}}{\mu^{2}}
$$

where $C_{i}=C_{F}$ for quark-antiquark initiated processes and $C_{i}=C_{A}$ for gluon-gluon initiated processes. For the choice $\mu \sim q_{T}$, as appropriate for the beam functions, we should count $\eta_{i} \sim 1$. In ref. [24] the role of the anomaly exponent inside the $x_{\perp}$ integral at very small $q_{T}$ was analyzed in detail. Instead of the Fourier exponential, the large $x_{\perp}$ behavior of the integrand is driven by the anomaly and by the double logarithms $L_{\perp}^{2}$ inside the beam function. In the limit $q_{T} \rightarrow 0$, the $x_{\perp}$ integral becomes Gaussian and can be 
analyzed with a saddle point approximation, an observation that was made very early by Parisi and Petronzio [75]. The appropriate value of $\mu$ in this limit is denoted by $q^{*}$ and given by the value for which $\eta_{i}$ becomes equal to one [24]. Consequently one has

$$
q^{*}=Q^{2} \exp \left(-\pi / C_{i} / \alpha_{s}\left(q^{*}\right)\right)
$$

and we solve for $q^{*}$ numerically in our setup for each integration "event". It is the characteristic scale of the process for very small $q_{T}$ and in practice well in the perturbative regime. The physical picture behind this formalism is that instead of soft radiation recoiling against the high- $Q^{2}$ system, the radiation for $q_{T} \rightarrow 0$ consists of QCD emissions at a scale $q^{*}$ recoiling against each other. For on-shell $Z$ production $q^{*}$ is about $2 \mathrm{GeV}$ and for Higgs production around $8 \mathrm{GeV}$.

To achieve uniform perturbative accuracy also for $q_{T} \rightarrow 0$, it has been observed that one should count $L_{\perp} \sim 1 / \sqrt{\alpha_{s}}$ [24]. This was called improved power counting to distinguish it from the standard counting $L_{\perp} \sim 1$ relevant at moderately small $q_{T}$. To implement this power counting, it is important to factor out the enhanced double-logarithmic part of the beam functions. To this end we work with the functions $\bar{B}_{i}$ which are defined through

$$
B_{i}\left(\xi_{i}, x_{\perp}, \mu\right)=e^{h_{i}\left(L_{\perp}, \alpha_{s}\right)} \bar{B}_{i}\left(\xi_{i}, x_{\perp}, \mu\right),
$$

where $h_{i}\left(L_{\perp}, \alpha_{s}\right)$ is provided by the solution of the RGE

$$
\frac{\mathrm{d}}{\mathrm{d} \log \mu} h_{i}\left(L_{\perp}, \alpha_{s}\right)=C_{i} \gamma_{\mathrm{cusp}} L_{\perp}-2 \gamma^{i}\left(\alpha_{s}\right),
$$

with boundary condition $h_{i}\left(L_{\perp}, \alpha_{s}\right)=0$. For the cusp anomalous dimension $\gamma_{\text {cusp }}$ and the quark and gluon anomalous dimensions $\gamma^{i}$ see refs. [69, 76]. The functions $\bar{B}_{i}$ are then implemented numerically in our code.

The modified beam functions $\bar{B}_{i}$ can be factorized further into a convolution

$$
\bar{B}_{i}\left(\xi, x_{\perp}, \mu\right)=\sum_{j} \int_{\xi}^{1} \frac{\mathrm{d} z}{z} \bar{I}_{i \leftarrow j}\left(z, x_{\perp}, \mu\right) f_{j}(\xi / z, \mu),
$$

of perturbative kernels $\bar{I}_{i \leftarrow j}\left(z, x_{\perp}, \mu\right)$ with the standard PDFs $f_{j}(\xi, \mu)$. For our resummation at $\mathrm{N}^{3} \mathrm{LL}$ we need the kernel function at two loops, which were computed in refs. [77, 78]. After the double-logarithmic part has been removed, the beam functions only depend polynomially on $L_{\perp}$. We are therefore able to perform the Fourier integral independently of the rest of the beam functions over the combined anomaly factor and the relevant powers of $L_{\perp}$.

In our code, we expand each individual ingredient to a common accuracy, according to our improved logarithmic and $\alpha_{s}$ power counting. Explicitly these are the hard function $\mathcal{H}$, the exponent of the hard function evolution $U$, the combined collinear anomaly and double-logarithmic exponent $h_{i}$, and the product of beam functions $B_{i} \cdot B_{j}$. Overall we achieve an accuracy of $\alpha_{s}$ relative to Born level for $\mathrm{N}^{2} \mathrm{LL}$ resummation and $\alpha_{s}^{2}$ relative to Born level for $\mathrm{N}^{3} \mathrm{LL}$ resummation, respectively. In the improved counting $\sqrt{\alpha_{s}} \sim 1 / L_{\perp} \sim \epsilon$, we include terms up to $\epsilon^{3}$. To also achieve higher accuracy for very small $q_{T} \rightarrow 0$, one 
would need to include additional terms in the beam functions. These terms are predicted by the RGE and were included in version two of the CuTe code which achieves $\epsilon^{5}$ accuracy. Numerically their effect is small.

Matching to fixed order. A simple additive matching prescription

$$
\left.\frac{\mathrm{d} \sigma^{\mathrm{N}^{3} \mathrm{LL}}}{\mathrm{d} q_{T}}\right|_{\text {naively matched to NNLO }}=\frac{\mathrm{d} \sigma^{\mathrm{N}^{3} \mathrm{LL}}}{\mathrm{d} q_{T}}+\underbrace{\frac{\mathrm{d} \sigma^{\mathrm{NNLO}}}{\mathrm{d} q_{T}}-\left.\frac{\mathrm{d} \sigma^{\mathrm{N}^{3} \mathrm{LL}}}{\mathrm{d} q_{T}}\right|_{\text {exp. to NNLO }}}_{\text {matching correction } \Delta \sigma}
$$

combines the resummed result at small $q_{T}$ with the fixed-order predictions at larger $q_{T}$, but suffers from two problems. First of all, the fixed-order result is only recovered up to higher-order terms. While formally not a problem, the leftover higher-order terms can induce unphysical behavior. We should therefore switch off the resummation at large $q_{T}$, which we implement using a transition function $t(x)$ with $x=q_{T}^{2} / Q^{2}$. This function is constructed so that $t(x)=1+\mathcal{O}(x)$ near $x=0$ and $t(x \geq 1)=0$. The intermediate behavior is such that it smoothly switches the resummation off as $x \rightarrow 1$. A similar problem arises for small $q_{T}$. The matching corrections are power suppressed, but can become numerically unstable and suffer from large unresummed logarithms. For this reason, we switch the matching off at very small $q_{T}$, below a cutoff scale $q_{0} \lesssim 1 \mathrm{GeV}$. The following modified matching prescription

$$
\left.\frac{\mathrm{d} \sigma^{\mathrm{N}^{3} \mathrm{LL}}}{\mathrm{d} q_{T}}\right|_{\text {matched to NNLO }}=t(x)\left(\frac{\mathrm{d} \sigma^{\mathrm{N}^{3} \mathrm{LL}}}{\mathrm{d} q_{T}}+\left.\Delta \sigma\right|_{q_{T}>q_{0}}\right)+(1-t(x)) \frac{\mathrm{d} \sigma^{\mathrm{NNLO}}}{\mathrm{d} q_{T}}
$$

addresses both issues discussed above. Since we match on the level of the differential cross section, the fully inclusive fixed-order result is only restored within the nominal perturbative accuracy, and not exactly. For inclusive $Z$ production it was found that the difference between resumming and matching the spectrum or the cumulant, which would preserve the integrated fixed-order result, are numerically small [24]. A detailed comparison of the two approaches can be found in ref. [79].

Choosing an appropriate transition region has to be done in dependence of the process and the kinematical cuts. This is necessary in order not to include resummation in a region where it is no longer valid. While it could be considered a drawback to have to manually choose the transition region and a transition function, we believe that it offers clear advantages: the transition is performed transparently and we can guarantee which parts of the fully matched resummation are included in which kinematical region. Contributions where the $q_{T}$ resummation clearly becomes invalid, for example due to kinematical thresholds, can be fully excluded.

Below, we discuss the matching procedure in detail for the diboson processes $\gamma \gamma$ and $Z \gamma$ where kinematical thresholds require switching off the resummation relatively early. To choose the transition region, we first evaluate the size of the matching corrections relative to the (naively) matched result for each process and set of cuts. These relative corrections should be small in the resummation region, at worst of order one. Comparing results, we 
then try to identify a matching window in which the resummed and fixed-order results agree well enough that the transition between them can be performed reliably.

Within our setup one can easily implement any desired transition function or even implement other matching procedures. All our results in this study are obtained with a suitably parametrized sigmoid function. Following a choice in CuTe, we first define

$$
s(x ; l, r, u)=\left(1+\exp \left(\log \left(\frac{1-u}{u}\right) \frac{x-m}{w}\right)\right)^{-1}, \quad m=(r+l) / 2, \quad w=(r-l) / 2 .
$$

The function $s(x)$, parametrized by $l, r, u$, is defined to be $s(l)=1-u$ and $s(r)=u$. In terms of this sigmoid, our transition function $t\left(x ; x^{\min }, x^{\max }, u\right)$, where $x=q_{T}^{2} / Q^{2}$, is then defined by

$$
t\left(x ; x^{\min }, x^{\max }, u\right)=\left\{\begin{array}{ll}
1, & \text { for } x<x^{\min } \\
\frac{s\left(x ; x^{\min }, x^{\max }, u\right)}{s\left(x^{\min } ; x^{\min }, x^{\max }, u\right)}, & \text { otherwise }
\end{array}\right\} .
$$

This ensures that below $x^{\min }=\left(q_{T}^{\min } / Q\right)^{2}$ only the naively matched result is used, and at $x^{\max }$ for small $u \ll 1$ the transition function is approximately $u$. In practice it makes sense to set the transition function to zero below a small threshold like $10^{-3}$ without a noticeable discontinuity. This has the advantage that the deteriorating resummation and matching corrections do not impact the region of large $q_{T}$ at all. Our default choices in the remainder of this paper are $x^{\mathrm{min}}=0.001$, and $u=0.001$.

For the fiducial results studied here, we find that without the presence of a threshold or presence of photons, power-suppressed corrections are of order $q_{T}^{2} / Q^{2}$, and the size of the matching corrections is well-behaved up to relatively large values of $q_{T}^{2} / Q^{2}$. Concretely, we find that values of $x^{\max }=0.4$ and $x^{\max }=0.6$ can be used and allow us to estimate the effect of the matching. For the processes with photons and with experimental cuts inducing additional thresholds, we have to start the transition much sooner. This is discussed in detail in the sections for the $\gamma \gamma$ and $Z \gamma$ predictions. We plot all transition functions used in our study in figure 1.

Power corrections and recoil effects. The factorization theorem in eq. (2.1) is derived strictly in the limit $q_{T} \rightarrow 0$ and is subject to power corrections that scale like $q_{T}^{2} / Q^{2}$ for fully inclusive production of a large- $Q^{2}$ system. Through the matching to fixed-order predictions, the power corrections are automatically included to all powers in $q_{T} / Q$, but of course not resummed. Since the factorization theorem is a function of $q_{T}^{2}$, it is most natural to consider the cross section $d \sigma / d q_{T}^{2}$. In fixed-order perturbation theory, the inclusive cross section for $q_{T}>0$ takes the form

$$
\frac{d \sigma}{d q_{T}^{2}}=\frac{A}{q_{T}^{2}}+B+\ldots
$$

where the coefficients $A$ and $B$ depend logarithmically on $q_{T}^{2}$. The leading logarithms at the $n$-th order in these coefficients have the form $\alpha_{s}^{n}(\mu) \ln ^{2 n-1}\left(q_{T}^{2} / \mu^{2}\right)$. The terms contained 


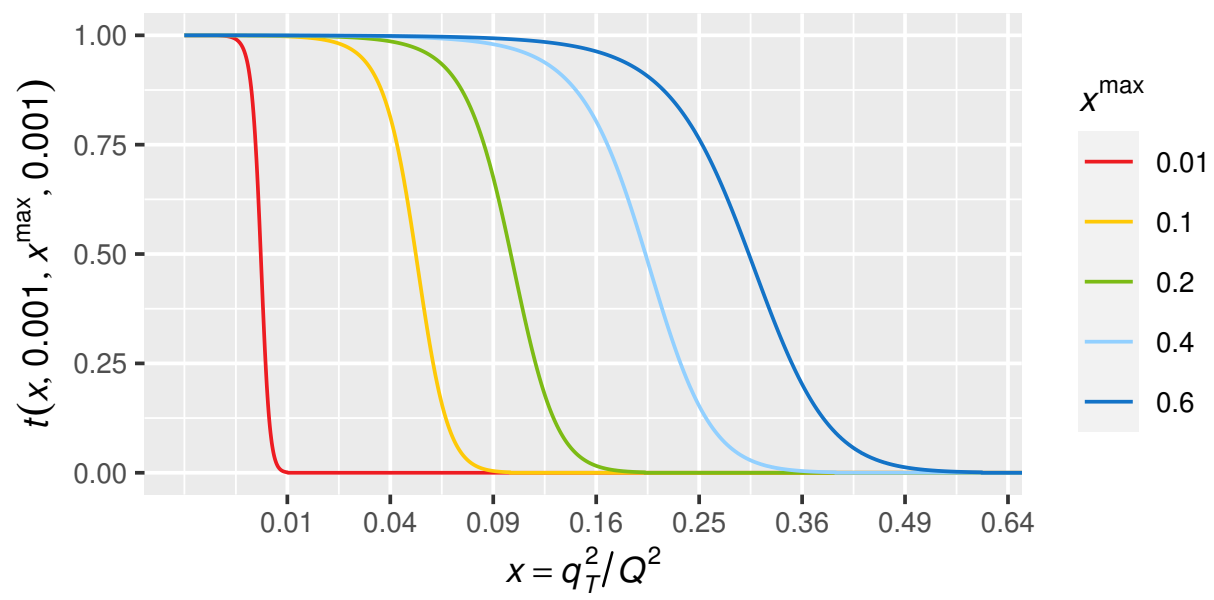

Figure 1. The transition function defined in (2.9) for different values of the parameter $x^{\max }$ which determines the position of the transition. The $x$-axis is displayed on a square-root scale to guide the eye on the quadratic $q_{T}$-dependence.

in $A$ are captured by the factorization formula, while the contributions in $B$ and all other power-suppressed terms are added through the matching correction $\Delta \sigma$.

Resummation cures the divergence of the cross section and the quantity $d \sigma / d q_{T}^{2}$ takes a finite value for $q_{T} \rightarrow 0$. A detailed discussion of the intercept for $q_{T} \rightarrow 0$ was given in ref. [24]; in this context the $\epsilon$-expansion discussed earlier plays a crucial role. Much less is known about effect of resummation on the power corrections, but first leadinglogarithmic resummed results for power-suppressed contributions indicate that Sudakov suppression is present also in this case [80-85]. Since we do not resum the power-suppressed matching corrections, their computation becomes unreliable at low $q_{T}$ because higher-order terms are enhanced by large logarithms and they can start to numerically compete with the resummed, Sudakov-suppressed leading-power cross section for $q_{T} \rightarrow 0$. We should therefore switch off the matching at very low $q_{T}$, which is achieved using a hard cutoff $q_{T}>q_{0}$ in eq. (2.8). This is also necessary for numerical stability, as we will discuss in detail in section 3 .

While the power corrections are quadratic in $q_{T}$ for the inclusive cross section, cuts on the leptonic final state can induce enhanced power corrections, depending on the treatment of kinematics in the hard function. To understand this effect, consider $Z$ production. Since the factorization theorem is valid in the limit $q_{T} \rightarrow 0$ and the leptons from the $Z$ decay have hard momenta, we can formally neglect the transverse momentum in the hard function and evaluate it with Born kinematics. Of course, after the expansion the two leptons then have vanishing total transverse momentum, so that it is no longer possible to directly access $q_{T}$ through the leptons. We can however choose one of the two lepton momenta, for example $p_{1}$, from the Born-level results and then define the momentum of the other one via momentum conservation as $p_{2}=q-p_{1}$. After this, we can again obtain $q_{T}$ and related observables through the lepton momenta and we can also impose fiducial cuts on the leptons. While this procedure is correct in the limit $q_{T}$, it is not unique and it was pointed out in ref. [40] 
that the ambiguity in this prescription corresponds to a $\mathcal{O}\left(q_{T} / Q\right)$ power correction. In ref. [86] it was explicitly demonstrated that the specific prescriptions discussed above leads to linear power corrections in the presence of fiducial cuts on the lepton momenta, even for azimuthally symmetric observables. Of course, it is not necessary to approximate the lepton kinematics and through a careful analysis of the lepton and hadron tensors it was recently demonstrated in ref. [45] that working with the exact lepton tensor avoids the occurrence of such linear power corrections.

The analysis in ref. [45] is based on decomposing and analyzing the lepton and hadron tensors, which would become very involved for multi-boson production. But the physical picture behind this analysis is simple: linear power corrections can be avoided by keeping the exact kinematics in the hard function. The easiest way to achieve this is to perform the factorization analysis in a frame, where the total transverse momentum of the decay products is zero so that no expansion is performed in the hard function and the decay kinematics are not altered by the expansion because it is equal to the Born-level kinematics.

In our code we achieve this, by generating the hard function with Born-level kinematics and then performing a Lorentz-boost into the lab frame, where the bosons carry the appropriate transverse momentum. More specifically, following ref. [26], we start by generating the Born-level phase space and then boost this system to have transverse components $\left(q_{T} \cos \phi, q_{T} \sin \phi\right)$, where we now additionally integrate over the values of $q_{T} \geq 0$ and $\phi \in[0,2 \pi]$ using Monte Carlo methods. We use the boosted momenta to evaluate the Born matrix elements and hard function and to perform the kinematical cuts. In the language of ref. [40], this corresponds to a specific recoil prescription, but the advantage of using a boost is that we keep the exact hard kinematics. While we will not attempt to formally prove that our procedure avoids the presence of linear power corrections in azimuthally symmetric observables, we have numerically verified that such corrections are absent for the fiducial cuts used in Drell-Yan measurements.

Finally, let us stress that our factorization theorem is fully differential in $q^{\mu}$ and the electroweak momenta. For this reason, we can not only analyze the transverse momentum spectrum $q_{T}$, but also related observables. For example, in this study we also present resummed results for $\phi^{*}[2]$ in the limit $\phi^{*} \rightarrow 0$, and the azimuthal angle for $\Delta \Phi \rightarrow \pi$. Of course to access such obervables, which are defined purely in terms of electroweak momenta, one either needs to use momentum conservation to recast them in terms of $q^{\mu}$ and hard momenta as was done in refs. [87-89], or one needs to a adopt prescription to account for the recoil, as we do by boosting. These prescriptions are equivalent at leading power, but our specific choice avoids the presence of linear power corrections. When analyzing observables, such as $\phi^{*}$ or $\Delta \Phi$, we could choose to parametrize the transition function (2.9) in terms of these, but for simplicity we always write it as a function of $q_{T}^{2} / Q^{2}$.

Enhanced power corrections from photon isolation. To separate direct photon production from photons arising in hadron decays, experiments impose that photons should be isolated from hadronic radiation. More precisely, only low- $q_{T}$ hadronic radiation is allowed inside a cone around the photon. In the limit $q_{T} \rightarrow 0$, and at leading power, photons are automatically isolated since all radiation has low $q_{T}$. This implies that the 

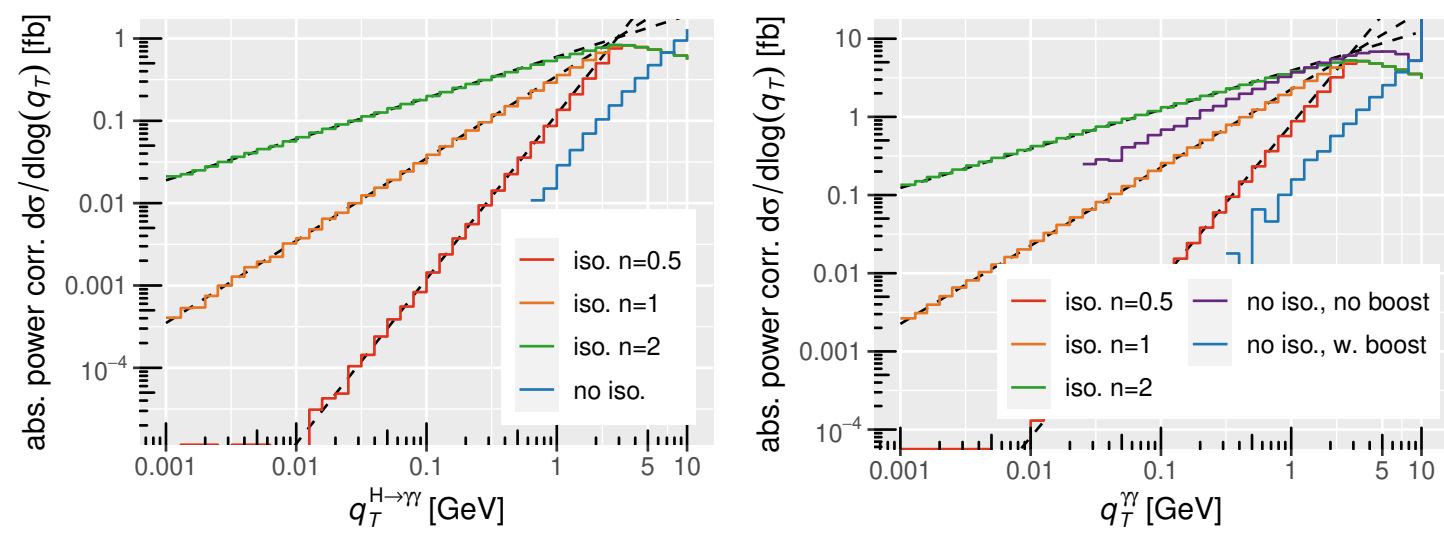

Figure 2. Power-suppressed matching corrections for $p p \rightarrow H \rightarrow \gamma \gamma$ (left) and $p p \rightarrow \gamma \gamma$ (right). For diphoton production only the $u \bar{u}$ channel is shown, with cuts $q_{T}^{\gamma}>25 \mathrm{GeV}$ on both photons. We plot results for the values $n=0.5,1,2$ of the isolation parameter $n$. Power corrections not from photon isolation are shown as purple and blue lines. The dashed lines show the scaling $\left(q_{T} / Q\right)^{1 / n}$ derived in ref. [86].

leading-power factorization theorem (2.1) applies also to processes with photons in the final state.

The photon isolation induces subleading power corrections, that are included via the matching to fixed-order predictions. However, the nature and size of these power corrections is different from what we encountered earlier since they are not imposed on the electroweak final state, but directly affect the hadronic matrix elements.

For our studies of processes with photons, we adopt the smooth-cone isolation introduced by Frixione [90], which fully suppresses the collinear singularity from the $q \rightarrow q \gamma$ splitting in an infrared-safe way, eliminating the need for fragmentation functions. It restricts the transverse energy inside a cone of size $R$ to

$$
E_{T}^{\mathrm{had}} \equiv \sum_{j: d(j, \gamma) \leq r} E_{T}^{j} \leq E_{T}^{\gamma, \max } \chi(r) \quad \forall r<R
$$

where $d(i, j)=\sqrt{\left(\phi_{i}-\phi_{j}\right)^{2}+\left(\eta_{i}-\eta_{j}\right)^{2}}$ is the separation in azimuthal angle $\phi$ and rapidity $\eta$ between parton $i$ and photon $j$. The angular function is

$$
\chi(r)=\left(\frac{1-\cos r}{1-\cos R}\right)^{n} \approx\left(\frac{r^{2}}{R^{2}}\right)^{n},
$$

where the approximation is valid for $R \ll 1$. The isolation energy $E_{T}^{\gamma, \max }$ can either be a fixed value or a fraction $\epsilon$ of the total photon transverse energy $E_{T}^{\gamma, \max }=\epsilon E_{T}^{\gamma}=\epsilon q_{T}^{\gamma}$.

The effects of photon isolation on power-suppressed terms in $q_{T}$ factorization have been studied in ref. [86]. These authors considered inclusive $H \rightarrow \gamma \gamma$ production with photon isolation cuts and inclusive diphoton production restricted to the $q \bar{q}$ channel with photon isolation cuts and photon $q_{T}$ cuts. They showed that the smooth-cone isolation requirement induces subleading terms scaling as $\left(q_{T} / Q\right)^{1 / n}$, where $n>0$ is the parameter in the isolation prescription above. We study this dependence in figure 2 for $H \rightarrow \gamma \gamma$ 
and diphoton production. Our numerical results nicely confirm this scaling of the power corrections. For comparison we also show the matching corrections not associated with photon isolation as blue and purple lines in figure 2. For $p p \rightarrow \gamma \gamma$ (right panel) we impose $q_{T}^{\gamma}>25 \mathrm{GeV}$. For the purple lines, Born-level kinematics are used for the photons, while for the blue one the recoil is taken into account using the boost prescription detailed above. We observe that these power corrections scale as the first power without the boost and as the second power with the boost. The recoil does not play a role for $p p \rightarrow H \rightarrow \gamma \gamma$ (left panel) since no fiducial cuts are employed.

While we reproduce the result of ref. [86] for the $q \bar{q}$ partonic channel, we observe a different behavior if all partonic channels are included, due to fragmentation contributions. To explain the difference we consider the emission of a single soft particle with momentum $k$ into the isolation cone. For one emission $q_{T}=k_{T}$ so that the value of the transverse momentum of the particle is fixed. The momentum dependence of the squared, spin averaged amplitudes for soft gluon and soft quark emission are

$$
\left|\mathcal{M}_{g}\right|^{2} \propto \frac{p_{1} \cdot p_{2}}{p_{1} \cdot k p_{2} \cdot k}=\frac{1}{k_{T}^{2}} \quad \text { and } \quad\left|\mathcal{M}_{q}\right|^{2} \propto \frac{1}{2 p_{\gamma} \cdot k}
$$

respectively. The momenta $p_{1}$ and $p_{2}$ are the momenta of the incoming partons and $p_{\gamma}$ is the photon momentum which defines the isolation cone. The result (2.13) shows that gluon emission is a leading-power effect while soft quark emissions are suppressed by one power of $k_{T}$. Writing the phase-space integral in terms of the transverse momentum, rapidity and azimuthal angle,

$$
\frac{\mathrm{d}^{3} k}{E_{k}}=\mathrm{d} k_{T} k_{T} \mathrm{~d} y \mathrm{~d} \phi
$$

we see that the soft gluon emission suffers from a soft divergence, while the quark emission has a collinear pole, which is regularized by the smooth-cone isolation requirement (2.11). For fixed transverse momentum $k_{T}$ and $R \ll 1$, the isolation requirement implies that the angular distance $r^{2}=d(k, \gamma)^{2}=\Delta y^{2}+\Delta \phi^{2}$ must fulfill

$$
r^{2} \geq R_{\min }^{2}=R^{2}\left(\frac{k_{T}}{E_{T}^{\gamma, \max }}\right)^{\frac{1}{n}} .
$$

The emitted particle can thus no longer be exactly collinear to the photon.

With these considerations we can now easily evaluate the power corrections associated with gluon and quark emission. Gluon emission is a leading-power effect and the power corrections are obtained by evaluating the difference between the isolated case and the inclusive production

$$
\begin{aligned}
\Delta \frac{\mathrm{d} \sigma}{\mathrm{d} q_{T}^{2}} & \propto \int \mathrm{d} y \mathrm{~d} \phi\left[\theta\left(r-R_{\min }\right)-1\right]\left|\mathcal{M}_{g}\right|^{2} \\
& =-\frac{1}{q_{T}^{2}} \int_{0}^{R_{\min }} \mathrm{d} r r=-\frac{R_{\min }^{2}}{q_{T}^{2}}=-\frac{R^{2}}{q_{T}^{2}}\left(\frac{q_{T}}{E_{T}^{\gamma, \max }}\right)^{\frac{1}{n}} .
\end{aligned}
$$

This reproduces the result of ref. [86]. Next, let us turn to fragmentation. In this case, the entire effect is a power correction, so we evaluate

$$
\Delta \frac{d \sigma}{\mathrm{d} q_{T}^{2}} \propto \int_{R_{\min }}^{R} \mathrm{~d} r r\left|\mathcal{M}_{q}\right|^{2} \approx \frac{1}{q_{T} p_{T}^{\gamma}} \int_{R_{\min }}^{R} \frac{\mathrm{d} r}{r}=\frac{1}{q_{T} p_{T}^{\gamma}} \ln \frac{R}{R_{\min }},
$$


where we approximated $2 p_{\gamma} \cdot k=k_{T} p_{T}^{\gamma} r^{2}+O\left(r^{4}\right)$. Here the dependence on the isolation requirement is logarithmic and the power correction is always first order. Furthermore, the effect in the gluon channel is suppressed by the cone radius $R^{2}$, while there is no such suppression in the fragmentation case. First-order power corrections will thus always be present and for small cone radius they will numerically dominate over the gluonic power corrections, even if these are larger than first order for $n>1$. We will present numerical results for the matching corrections for the sum of all partonic channels and including the fiducial cuts on the photons in section 3. The results in this section will confirm the presence of first-order power corrections.

Linear or stronger power corrections lead to matching corrections which tend to a constant or even grow in $\mathrm{d} \sigma / \mathrm{d} q_{T}$ for $q_{T} \rightarrow 0$ and overwhelm the resummed leading-power result. This implies that it is not possible to obtain reliable predictions for very small $q_{T}$ in such cases, at least not without resumming also the power corrections. We will face this problem in section 3 when studying processes with photons in the final state.

Having discussed the effect of photon isolation on power-suppressed corrections at small $q_{T}$, we should mention that photon isolation also leads to logarithmically enhanced contributions at large transverse momentum, since there is then a region of phase space, where the radiation is restricted by the isolation requirement. This is a typical situation in which non-global logarithms arise [91] and their numerical effect in photon-production cross sections was studied in ref. [92] at leading-logarithmic accuracy. While the argument of the logarithms is large for the experimentally imposed photon isolation energies, the effect on the cross section is moderate, since it is suppressed by $R^{2}$. Similar conclusions were reached in ref. [59], which studied their size for the $Z \gamma$ transverse-momentum spectrum.

Implementation. We have implemented the presented framework in a modular Fortran 2008 code, where hard function evolution, beam functions and Fourier integrals are calculated separately and assembled to the designated order for resummed result and its fixed-order expansion. All components are combined with an easy to modify transition function in the MCFM plotting routines. The phase-space parametrization routine for each process allows for an efficient integration down to very small $q_{T}$. Since the essential resummation pieces are only loosely coupled to MCFM, they could easily be reused or integrated into other codes, for example as a direct stand-alone extension of the interface to event files [26] to $\mathrm{N}^{3} \mathrm{LL}$ and gluon-gluon initiated processes.

Resummation parameters that can and should be changed in the input file during normal use are the integration range for the resummation and its expansion, and the hard cutoff below which the matching corrections are turned off. Further details on how to use the code will be made available in the manual together with the code.

The NNLO processes available to be matched with $\mathrm{N}^{3} \mathrm{LL}$ resummation are $H, Z, W^{ \pm}$[19], $W^{ \pm} H, Z H$ [20], $\gamma \gamma$ [93] and $Z \gamma$ [21]. Since MCFM implements several more processes at NLO, these could easily be matched with $\mathrm{N}^{2} \mathrm{LL}$ resummation and we would be happy to add these by request. All processes include all leptonic decay channels and Higgs production includes all major decay channels. Furthermore, for $Z$ production electroweak corrections have been implemented [94]. 
Estimation of perturbative truncation uncertainty. We estimate the perturbative truncation uncertainty by varying the hard, renormalization, factorization and resummation scales in our calculation with the two multipliers

$$
\left(k_{F} ; k_{R}\right) \in\{(2,2),(0.5,0.5),(2,1),(1,1),(0.5,1),(1,2),(1,0.5)\} .
$$

Since we only consider two independent multipliers, some variations are correlated as detailed in the following. For the fixed-order computation and the matching correction we use $\mu_{F}=k_{F} \hat{Q}$ and $\mu_{R}=k_{R} \hat{Q}$. We choose the default hard scale as $\hat{Q}=Q$ for the benchmark comparison to CuTe, while we also use $\hat{Q}=\sqrt{Q^{2}+q_{T}^{2}}$ in other cases, in line with the choice typically made in fixed-order computations at larger $q_{T}$. The seven-point prescription for scale variation resulting from eq. (2.18) is common practice in the fixed-order community. To set the resummation scale, we first calculate $q^{*}$ for each integration phase-space point ("event") and then set

$$
\mu=\max \left(k_{F} \cdot\left(q_{T}+q^{*} \exp \left(-q_{T} / q^{*}\right)\right), 2 \mathrm{GeV}\right) .
$$

This choice ensures that the scale is always in a perturbative and numerically stable regime, and for very small $q_{T}$ approaches $q^{*}$, while otherwise smoothly transitioning to $q_{T}$. For the hard scale, we use $\mu_{h}=k_{R} \hat{Q}$. With this prescription, we avoid the introduction of four different multipliers at the price of correlating some variations in the matching correction and the resummed result.

At small $q_{T}$ the logarithms resummed up to $\mathrm{N}^{3} \mathrm{LL}$ dominate, and with the choice in eq. (2.19) the residual scale dependence can become small at very small $q_{T}$. In this region the problem arises that varying the resummation scale leads to very low values of $\mu$ for which the Fourier-integral becomes numerically unstable. To avoid this, we have set a minimum value of $\mu=2 \mathrm{GeV}$ in eq. (2.19), which restricts the scale variation but ensures that the scale $\mu$ always remains in the perturbative regime.

A drawback of this approach is that at very small $q_{T}$ of a few $\mathrm{GeV}$ the downwards variation for the resummation scale vanishes. To address this, one could symmetrize the uncertainties, if large asymmetries at small $q_{T}$ are observed. We find this not to be an issue with fiducial cuts since in this case the variation of the hard renormalization scale generates the bulk of the scale uncertainty. Furthermore, the overall uncertainty budget at such low values of $q_{T}$ should include non-perturbative effects that are not quantified here. Beyond that, various approaches have been used in the literature that argue for modifying the scale variation procedure in combination with resummation [56, 95]. Also in our case further variations could be considered. In addition to introducing a scale to estimate uncertainties from different exponentiations of the rapidity logarithms, we could, for example, introduce an additional evolution step to separate the scale in the perturbative kernels $\bar{I}_{i \leftarrow j}$ in the beam functions in eq. (2.6) from the PDF scale and then also vary this scale. Of course, ultimately one should simply compute the higher-order corrections to know their size.

Beam-function grids. While our setup can compute the beam functions on the fly by evaluating the convolution in eq. (2.6) with the PDFs for the relevant values of $\xi$ and $\mu$, it is computationally expensive to do so. It is much more effective to pre-compute LHAPDF 
grids [96] for the beam functions. After doing so, the calculation of the resummed component is no longer more time consuming than the other components. For each individual PDF grid five beam function grids are generated corresponding to the beam function coefficients of different orders of $\alpha_{s}$ and $L_{\perp}$. The grid pre-computation is fully parallelized through OpenMP and MPI or Fortran Coarrays, and if PDF uncertainties are enabled the eigenvector or replica PDF set members can also be pre-computed accordingly. Through the infrastructure of MCFM-9, matched results with multiple PDF sets, including their respective uncertainties, can in this way be computed simultaneously.

Checks. We have extensively compared all of our resummation ingredients at $\mathrm{N}^{3} \mathrm{LL}$ against a private prototype implementation in Mathematica that resulted in the code CuTe [25] as well as against the $\mathrm{N}^{2} \mathrm{LL}$ implementation in ref. [26] and find full agreement.

Since our implementation is based on MCFM-9, which employs jettiness subtractions for the NNLO calculations [97, 98], all processes have been extensively checked and IR cancellations have already been demonstrated to be numerically stable down to the permille level.

For all our presented results we checked that the fixed-order expansion of the resummed result and the fixed-order predictions agree for $q_{T} \rightarrow 0$. We performed this check down to values of $0.01 \mathrm{GeV}$ with sub-permille precision in the cancellation, depending on the process and cuts; see the individual process studies presented in the next section. With that we implicitly also tested numerically that these leftover power corrections to our $q_{T}$ resummation scale as predicted: for fiducial processes without photons we find that the power corrections without boosted Born kinematics are $\mathcal{O}\left(q_{T} / Q\right)$, while they are quadratic with a boost. For processes with photons we can furthermore check the fixed-order result and our framework by testing that power corrections due to smooth-cone photon isolation scale as $\left(q_{T} / Q\right)^{1 / n}$, where $n>0$ is given as a parameter in the isolation prescription [86]. This asymptotic behavior sets in sufficiently below the photon isolation cone energy $E_{T}^{\gamma, \max }$, which is typically just a few $\mathrm{GeV}$.

We also compared our fully inclusive results against the CuTe code: by default $\mathrm{CuTe}$ makes a series of choices that lead to power-suppressed differences. For example, it takes into account a finite- $q_{T}$ modification of the phase-space. When setting the phase-space integration to use Born-level kinematics we find full agreement for fixed-order results in $W, Z$ and Higgs production as well as for the fixed-order expansion of the resummed result at $\mathrm{N}^{3} \mathrm{LL}$. For the resummed part, our results agree with $\mathrm{CuTe}$ at $\mathrm{N}^{3} \mathrm{LL}$ within the choices available in $\mathrm{CuTe}$ for the expansion of the improved power counting scheme, see the following section.

\section{Results}

In this section we present resummed and matched results for a wide range of electroweak final states. As a first step we perform benchmark computations for fully inclusive $Z$-boson and $H$-boson production and compare against the code CuTe. ${ }^{3}$ The code CuTe is restricted

\footnotetext{
${ }^{3} \mathrm{CuTe}$ is available at https://cute.hepforge.org/.
} 
to $Z, W$ and $H$ production and does not allow for fiducial cuts, but is based on the same formalism and ingredients as our implementation. While the ingredients were individually cross checked against $\mathrm{CuTe}$, the numerical results for the cross sections differ through terms beyond the accuracy of the calculation. These include power-suppressed effects associated with a different treatment of phase space in $\mathrm{CuTe}$, as well as higher-order perturbative effects from different ways of organising the expansion. Given the different scheme choices, it is interesting to quantify the resulting differences that, in principle, should be covered by scale uncertainties.

After this benchmarking exercise, we impose experimental fiducial cuts and directly compare with measurements from ATLAS and CMS. For $Z$ production we compare with studies at $8 \mathrm{TeV}[99]$ and $13 \mathrm{TeV}[100]$. For $W^{ \pm}$-boson production, we compare with a transverse-momentum measurement at $8 \mathrm{TeV}$ [101]. A high-precision theoretical description of the Higgs production process requires a careful inclusion of top-quark mass effects which go beyond the scope of this study. For the moment, we therefore present results in the strict heavy-top limit with fiducial cuts that are imposed in an experimental $H \rightarrow \gamma \gamma$ analysis. Currently the experimental uncertainties in the Higgs transverse-momentum spectrum are still quite large, but it would be interesting to perform a detailed theoretical analysis in the future. Finally, we turn to diboson processes. For diphoton production we show novel results at $\mathrm{N}^{3} \mathrm{LL}$ accuracy going beyond previous results at $\mathrm{N}^{2} \mathrm{LL}$. For $Z \gamma$ production we also present novel results at $\mathrm{N}^{3} \mathrm{LL}$ that improve upon previous results limited to fixed order.

In all cases we show fully matched $\mathrm{N}^{3} \mathrm{LL}+\mathrm{NNLO}$ results, but usually refrain from showing results at a lower order or their scale uncertainties. For large $q_{T}$ the lower-order results are only Born-level accurate and perturbative uncertainties are not properly estimated solely through the running of $\alpha_{s}(\mu)$ and the PDFs, without further intrinsic scale dependence from renormalized loop integrals. Typically the first results that can give reliable uncertainties at large $q_{T}$ are given by our $\mathrm{N}^{3} \mathrm{LL}+\mathrm{NNLO}$ predictions that include the fixedorder results at large $q_{T}$ at a subleading order in $\alpha_{s}$.

Our results in the following are presented for random selections of some NNLO PDF sets with a fixed value of $\alpha_{s}\left(m_{Z}\right)=0.118$ : ABMP16 [102], CT14 [103], CT18 [104], MMHT2014 [105], MSTW2008 (this has $\left.\alpha_{s}\left(m_{Z}\right)=0.117\right)$ [106], NNPDF30 [107] and NNPDF31 [108] interfaced to LHAPDF [96]. We also compute and compare the uncertainties associated with the different PDF sets.

\subsection{Benchmark calculations and comparison with $\mathrm{CuTe}$}

As mentioned above, the implementations of the resummation formula in $\mathrm{CuTe}$ and CuTe-MCFM differ: the default approach taken in CuTe is to combine hard function and its evolution factor into a common exponent and expand this exponent to a designated logarithmic accuracy in $\alpha_{s}$. This approach thus exponentiates the higher-order corrections to the hard function. CuTe also implements certain higher-order beam function contributions which are relevant to obtain $\epsilon^{5}$ accuracy in the improved counting at very low $q_{T}$, while we only achieve $\epsilon^{3}$ accuracy, see the discussion in section 2. A second difference arises because CuTe modifies the phase-space integral to include power-suppressed effects. 
For the parton momentum fractions $\xi_{1,2}=\sqrt{\tau} e^{ \pm Y}$ entering the beam functions CuTe uses $\tau=\left(Q^{2}+q_{T}^{2}\right) / s$, while we work with the Born-level result $\tau=Q^{2} / s$.

To compare with $\mathrm{CuTe}$ we have ensured that all physical input parameters agree and then checked that the fixed-order predictions and expansion of the resummed cross section agree. We work with the NNPDF31_nnlo_as_0118 PDF set at $\sqrt{s}=13 \mathrm{TeV}$. To compare the resummed results, we work at $\epsilon^{4}$ in the improved power counting in CuTe. This order resembles most closely our new implementation, since we include some terms beyond $\epsilon^{3}$. In CuTe-MCFM we integrate over $q_{T}$ and present the results bin-wise, while CuTe is limited to evaluating individual $q_{T}$ values. CuTe can also parametrize non-perturbative effects and has different transition functions to choose from, but here we are only interested in the subleading differences of the resummed results for benchmarking purposes.

We present benchmark results for $Z$ production as a quark-antiquark initiated process and for $H$ production for a gluon-gluon initiated process. The other processes available in $\mathrm{CuTe}-\mathrm{MCFM}$ are all based on the same resummation ingredients and only differ in the hard function and Born amplitudes.

\subsubsection{Inclusive Higgs production}

In figure 3 we compare the resummed result for inclusive Higgs production without fixedorder matching obtained with CuTe to our new implementation CuTe-MCFM. This comparison gives an indication of the uncertainties from subleading terms due to the different scheme choices. The first panel shows the absolute distribution, while the second panel shows the ratio to our $\mathrm{N}^{3} \mathrm{LL}$ resummed result where scale uncertainties are also included.

Overall the predictions of $\mathrm{CuTe}$ and $\mathrm{CuTe}-\mathrm{MCFM}$ are within mutual scale uncertainties up to $30 \mathrm{GeV}$. Central values are also are well compatible and captured within one to two times the scale-uncertainty band of our own $\mathrm{N}^{3} \mathrm{LL}$ prediction. The discrepancy beyond $30 \mathrm{GeV}$ between CuTe and CuTe-MCFM is solely due to the choice of $\tau=\left(Q^{2}+q_{T}^{2}\right) / s$ for the phase-space integral in CuTe. Below we analyze this difference in detail for $Z$ production. Scale variation does not provide an estimate of the size of these power-suppressed differences, but performing the matching to fixed order would largely eliminate them.

At large $q_{T}$ one observes almost zero scale uncertainties for CuTe if the expansion is performed strictly in the exponent. At the same time, one sees a significant increase in the scale uncertainties at tiny $q_{T}$, where also the improved expansion order plays a big role. It is perhaps a bit disconcerting that formally equivalent prescriptions give such different scale variation bands. It seems that there is an accidental cancellation of scale uncertainties at play, as evidenced by the fact that these uncertainties increase significantly when we impose fiducial cuts, see figure 12 below. We also observe that the $\mathrm{N}^{3} \mathrm{LL}$ results are outside of the $\mathrm{N}^{2} \mathrm{LL}$ result. This is a reflection of the well known fact that the Higgs cross section suffers from large perturbative corrections. If we instead considered the normalized distribution, the bands would overlap. The small scale uncertainties of CuTe-MCFM at tiny $q_{T}$ are a consequence of the choice in eq. (2.19) and not indicative of the true uncertainty, which would also need to include an estimate of non-perturbative effects.

Having discussed the resummation, we now illustrate the numerical difficulties in computing the matching corrections in figure 4. The top panel shows the fixed-order predictions 

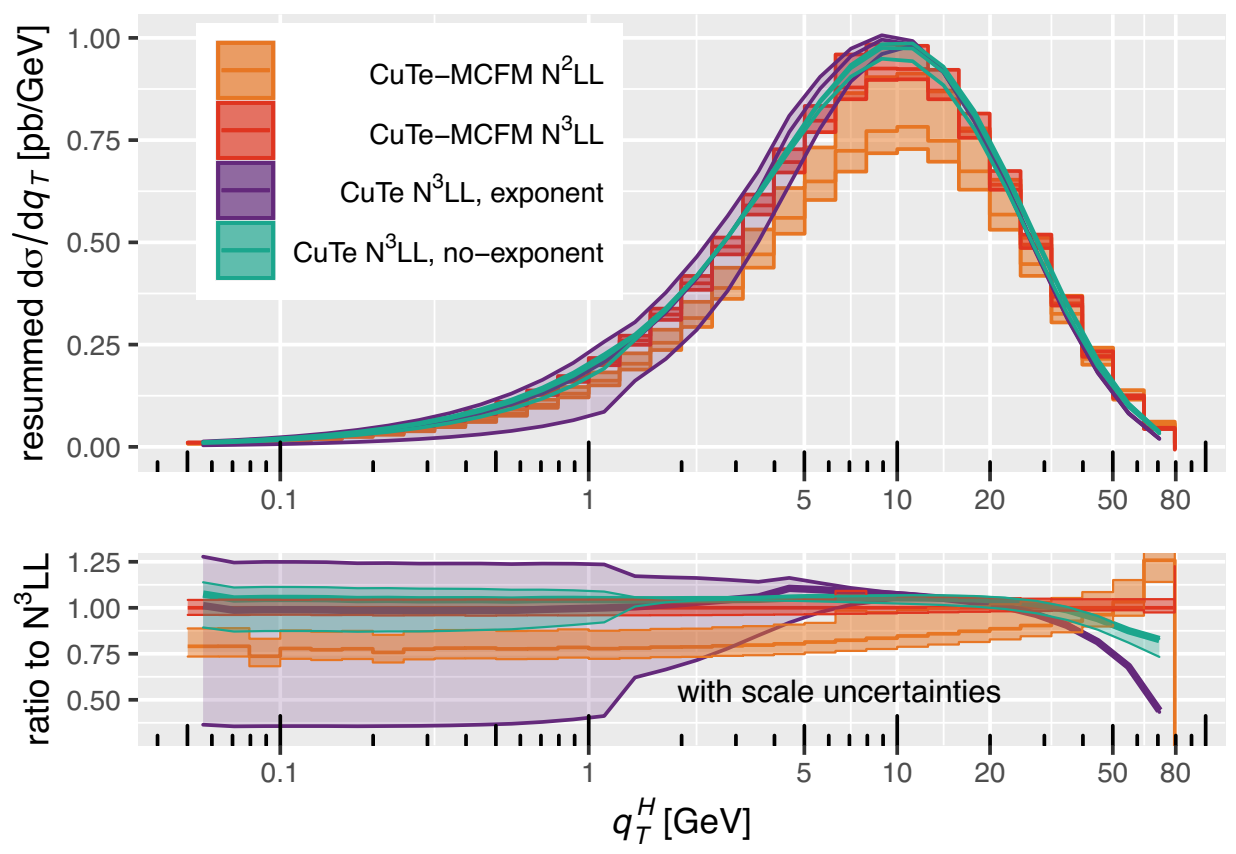

Figure 3. Resummed results without matching for inclusive Higgs production at $13 \mathrm{TeV}$ obtained using CuTe-MCFM and $\mathrm{CuTe}$ at different logarithmic orders. For CuTe we show results in two schemes: expanded in the exponent or on the level of the cross section. The shaded bands display scale uncertainties. The bottom panel shows the ratio to the $\mathrm{N}^{3} \mathrm{LL}$ result in CuTe-MCFM.

at $\alpha_{s}$ and $\alpha_{s}^{2}$ and their behavior towards $q_{T} \rightarrow 0$. Note that the $\alpha_{s}^{2}$ prediction has a zero around $2 \mathrm{GeV}$. The matching corrections are shown in second panel. Both cross sections are displayed as $\mathrm{d} \sigma / \mathrm{d} \log \left(q_{T}\right)=q_{T} \mathrm{~d} \sigma / d q_{T}=2 q_{T}^{2} \mathrm{~d} \sigma / d q_{T}^{2}$. Since the matching corrections are suppressed by $\mathcal{O}\left(q_{T}^{2}\right)$ they should decrease quadratically as $q_{T}$ is lowered and we indeed observe this behavior for moderately small $q_{T}$. However, the fixed-order result for $q_{T}^{2} \mathrm{~d} \sigma / d q_{T}^{2}$ and the fixed-order expansion of the resummed result both go to a constant in the same limit so that we encounter large numerical cancellations when computing the matching in the region of very small $q_{T}$.

Indeed the quadratic behavior of the matching corrections is spoiled by numerical problems for $q_{T} \lesssim 1 \mathrm{GeV}$. In this region one is limited by the Monte-Carlo integration, where, typically, relative uncertainties below $10^{-3}$ to $10^{-4}$ are computationally very expensive. Around $1 \mathrm{GeV}$ for the $\mathrm{N}^{2} \mathrm{LL}$ result, the cancellations in the computation of the matching correction already require a relative uncertainty of $10^{-4}$. The bottom panel in figure 4 shows that for $q_{T} \lesssim 1 \mathrm{GeV}$ the numerical noise in the matching corrections becomes large relative to the Sudakov suppressed resummed result.

Overall, the above considerations imply that, for practical numerical reasons alone, one has to turn off the matching corrections below a certain value of $q_{T}$ to not spoil the results at small $q_{T}$ with an incomplete cancellation. For observables with quadratic power corrections, imposing this cutoff is completely unproblematic, but we will revisit the issue when discussing processes with photons, where the power suppression is weaker. 

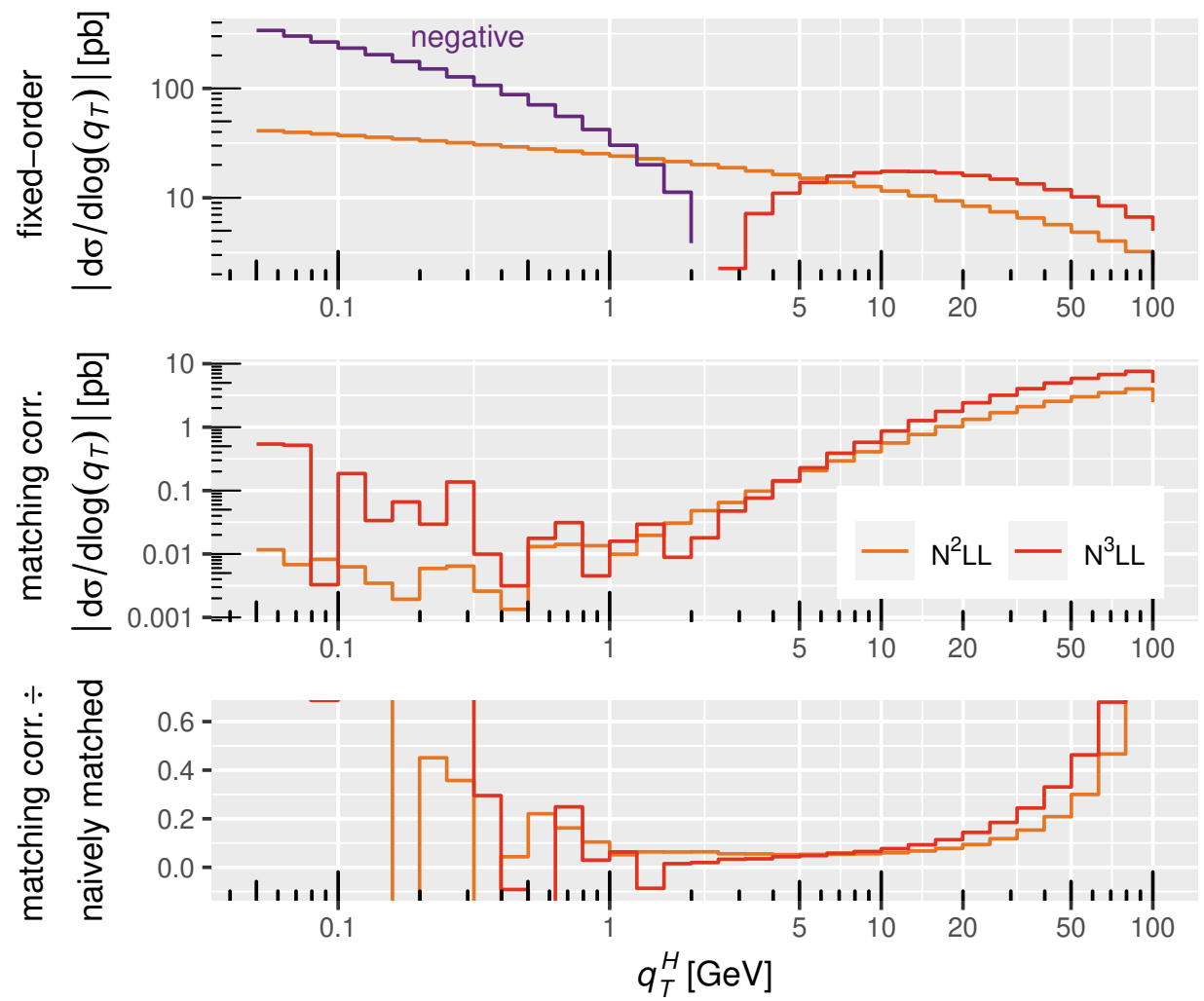

Figure 4. Top panel: fixed-order prediction at LO and NLO for inclusive Higgs production. Middle panel: absolute value of the matching corrections to show the cancellation towards $q_{T} \rightarrow 0$ and the resulting loss of numerical accuracy at very small $q_{T} \lesssim 1 \mathrm{GeV}$. Bottom panel: matching corrections relative to the naively matched result.

In any case, the computation of the power-suppressed matching terms using fixed-order perturbation theory is no longer viable in this region since the power corrections will involve large logarithms. On top of this, for such low values of $q_{T}$ also non-perturbative effects will play a role. For the remainder of this paper we switch off the matching corrections below $1 \mathrm{GeV}$ unless otherwise noted.

\subsubsection{Inclusive $Z$ production}

To benchmark a quark-antiquark initiated process we compare our fully inclusive predictions for $Z$ production with CuTe. The results for the fixed-order expansion and the matching are presented in figure 5 and are qualitatively similar to the ones for Higgs production in figure 4 . However, the matching corrections are significantly smaller and almost negligible below $10 \mathrm{GeV}$. Up to $50 \mathrm{GeV}$ they only reach few percent, but rapidly increase beyond that.

Next, let us look at the resummed results shown in figure 6. For smaller $q_{T}$, we observe good agreement between CuTe and CuTe-MCFM, but above $20 \mathrm{GeV}$ there is again no overlap within scale uncertainties with the results from CuTe. We have argued above that this is due to the inclusion of power-suppressed terms in the partonic momentum fractions in 

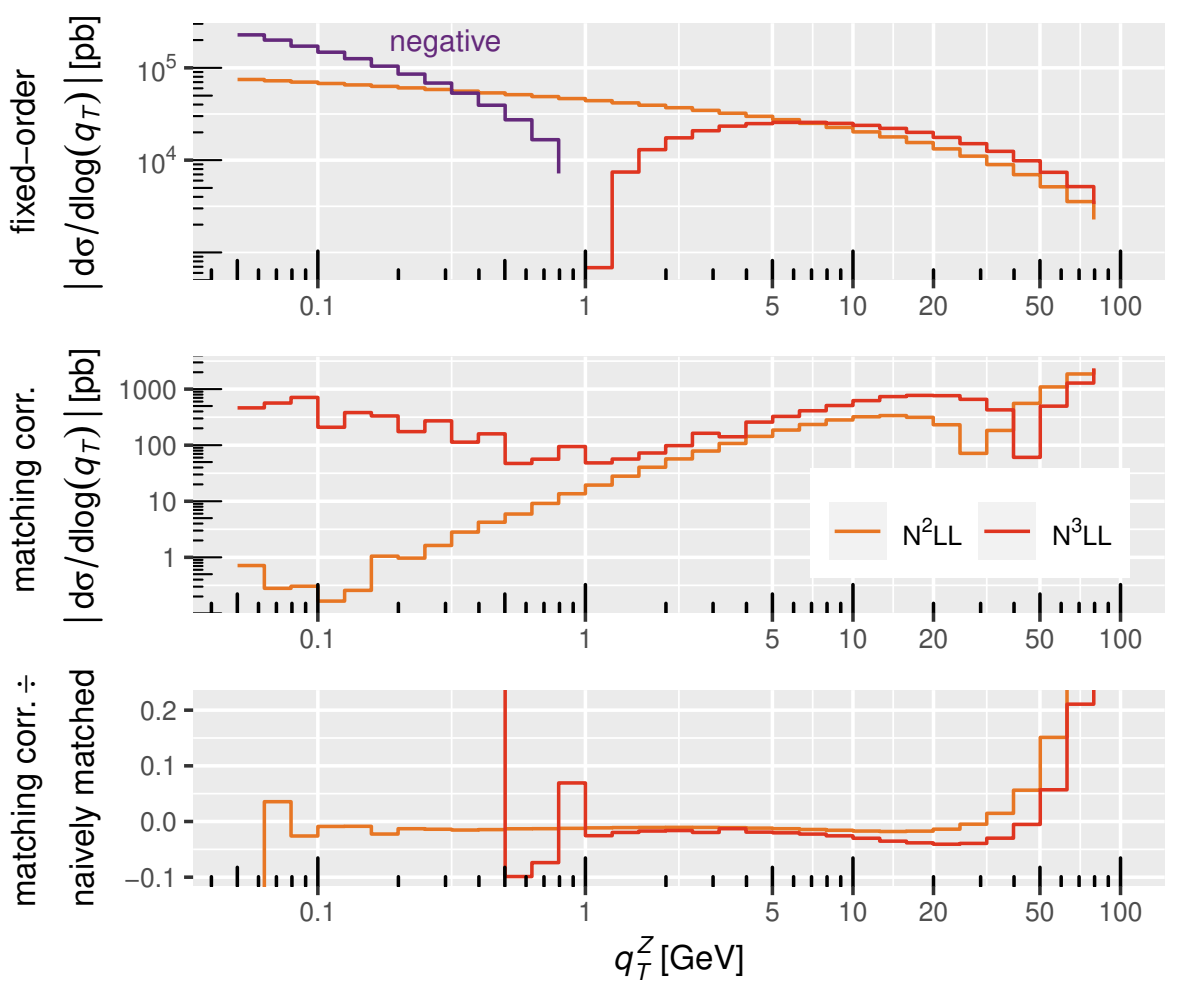

Figure 5. Top panel: fixed-order prediction at LO and NLO for inclusive $Z$ production. Middle panel: absolute matching corrections to show the cancellation towards $q_{T} \rightarrow 0$ and the effect of a limited numerical accuracy at small $q_{T}$. Bottom panel: matching corrections relative to the naively matched result.

CuTe, from setting $\tau \equiv \tau\left(q_{T}\right)=Q^{2}+q_{T}^{2}$. We verify this by including an additional curve where we have modified CuTe to switch off the suppressed terms $\tau\left(q_{T}=0\right)$ and find good agreement also at large $q_{T}$. While both schemes are valid, we observe that the one used in CuTe leads to larger matching corrections.

Since for $Z$ production the scale $q^{*}$ is about $2 \mathrm{GeV}$ and coincides with the minimum value of the resummation scale that we choose, the downwards scale variation becomes ineffective in our prescription below scales of $\sim 3 \mathrm{GeV}$. This can be clearly seen in the plot. An easy remedy would be to symmetrize the uncertainty band by taking the maximum of upper and lower variation, although the upwards variation can also become small with fiducial cuts. Similar as for the Higgs comparison, at $N^{3} \mathrm{LL}$ only the CuTe result with expansion in the exponent gives a sizable uncertainty below $1 \mathrm{GeV}$. On the other hand this uncertainty becomes unrealistically small above $5 \mathrm{GeV}$ compared to the CuTe-MCFM result.

\section{$3.2 \quad$ Fiducial $Z$ production}

We now turn to fiducial results, starting with $Z$ production, an experimental and theoretical standard candle. We compare with $Z \rightarrow l^{+} l^{-}$measurements presented in the $8 \mathrm{TeV}$ ATLAS study in ref. [99] and the $13 \mathrm{TeV}$ CMS study in ref. [100]. 

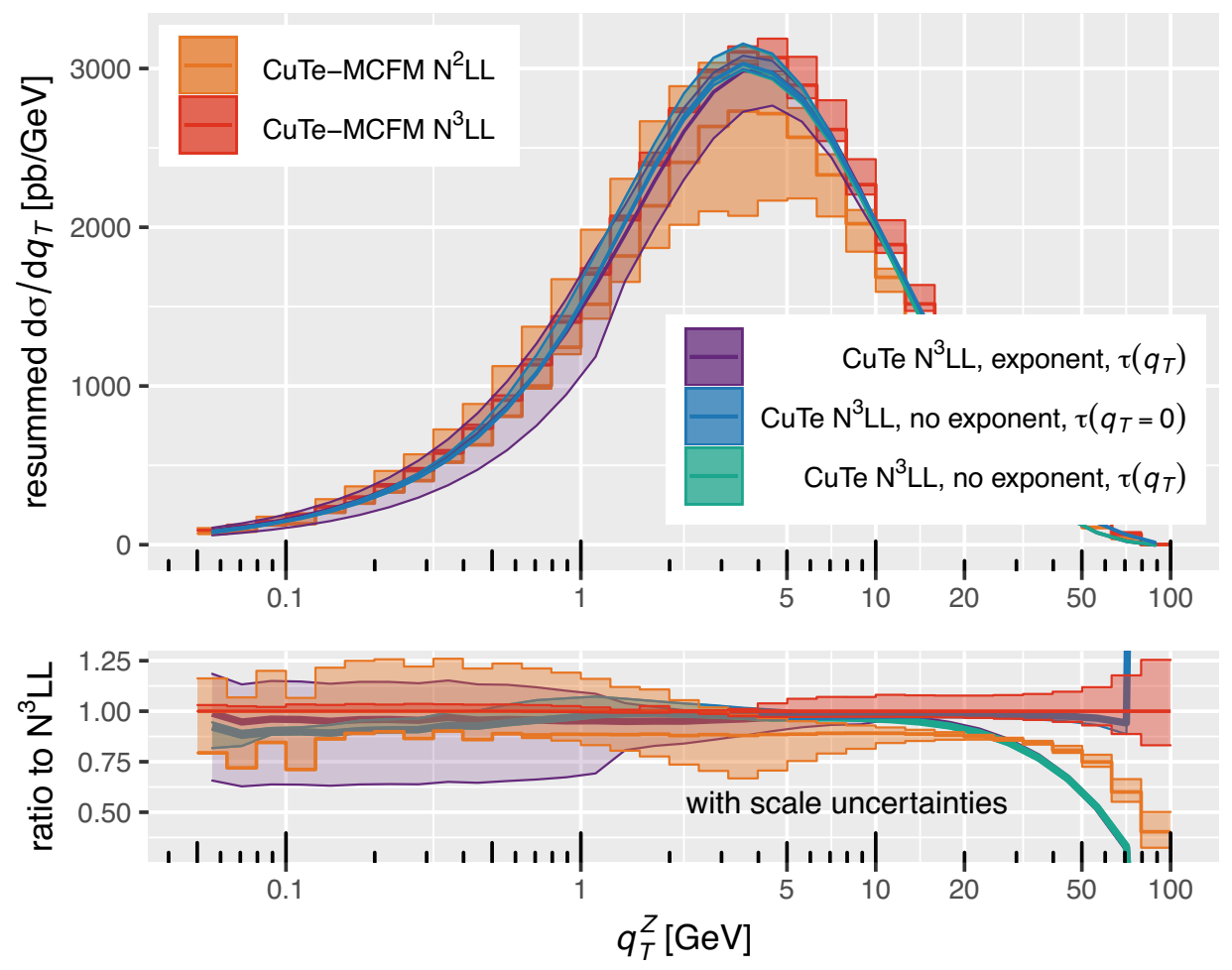

Figure 6. Resummed results without matching for inclusive $Z$ production at $13 \mathrm{TeV}$ obtained using CuTe-MCFM and CuTe at different logarithmic orders. For CuTe we show results in two schemes: expanded in the exponent or on the level of the cross section. We furthermore present results with two different treatments of power-suppressed terms in the phase space related to the choice of $\tau\left(q_{T}\right)$, see text. The shaded band displays scale uncertainties. The bottom panel shows the ratio to the $\mathrm{N}^{3} \mathrm{LL}$ result in CuTe-MCFM.

\begin{tabular}{|l|c|}
\hline Lepton cuts & $q_{T}^{l}>20 \mathrm{GeV},\left|\eta^{l}\right|<2.4$ \\
Separation cuts & $66 \mathrm{GeV}<m^{l^{+} l^{-}}<116 \mathrm{GeV},\left|y^{l^{+} l^{-}}\right|<2.4$ \\
\hline
\end{tabular}

Table 1. Fiducial cuts for $Z \rightarrow l^{+} l^{-}$at $\sqrt{s}=8 \mathrm{TeV}$, see ref. [99].

\subsubsection{ATLAS measurements at $8 \mathrm{TeV}$}

We first compare with the ATLAS $8 \mathrm{TeV}$ measurement [99], which imposes the cuts listed in table $1 .{ }^{4}$ All measurements are presented as normalized to the integrated fiducial cross section. Our predictions are calculated with a dynamic hard scale $\mu_{h}=\sqrt{Q^{2}+q_{T}^{2}}$ and the NNPDF31_nnlo_as_0118 PDF set.

In figure 7 we show our matched prediction in comparison with the measurement. We re-normalize all data to the $q_{T}$-integrated cross section with $q_{T}>2 \mathrm{GeV}$, since the first bin is likely to receive non-perturbative contributions that we do not model. Including the first bin for the normalization would therefore skew the results.

\footnotetext{
${ }^{4}$ As a side note, we strongly discourage the use of symmetric $q_{T}$ cuts, since this causes instabilities in higher-order calculations, and a slight asymmetry does not decrease the cross section much, see ref. [22].
} 


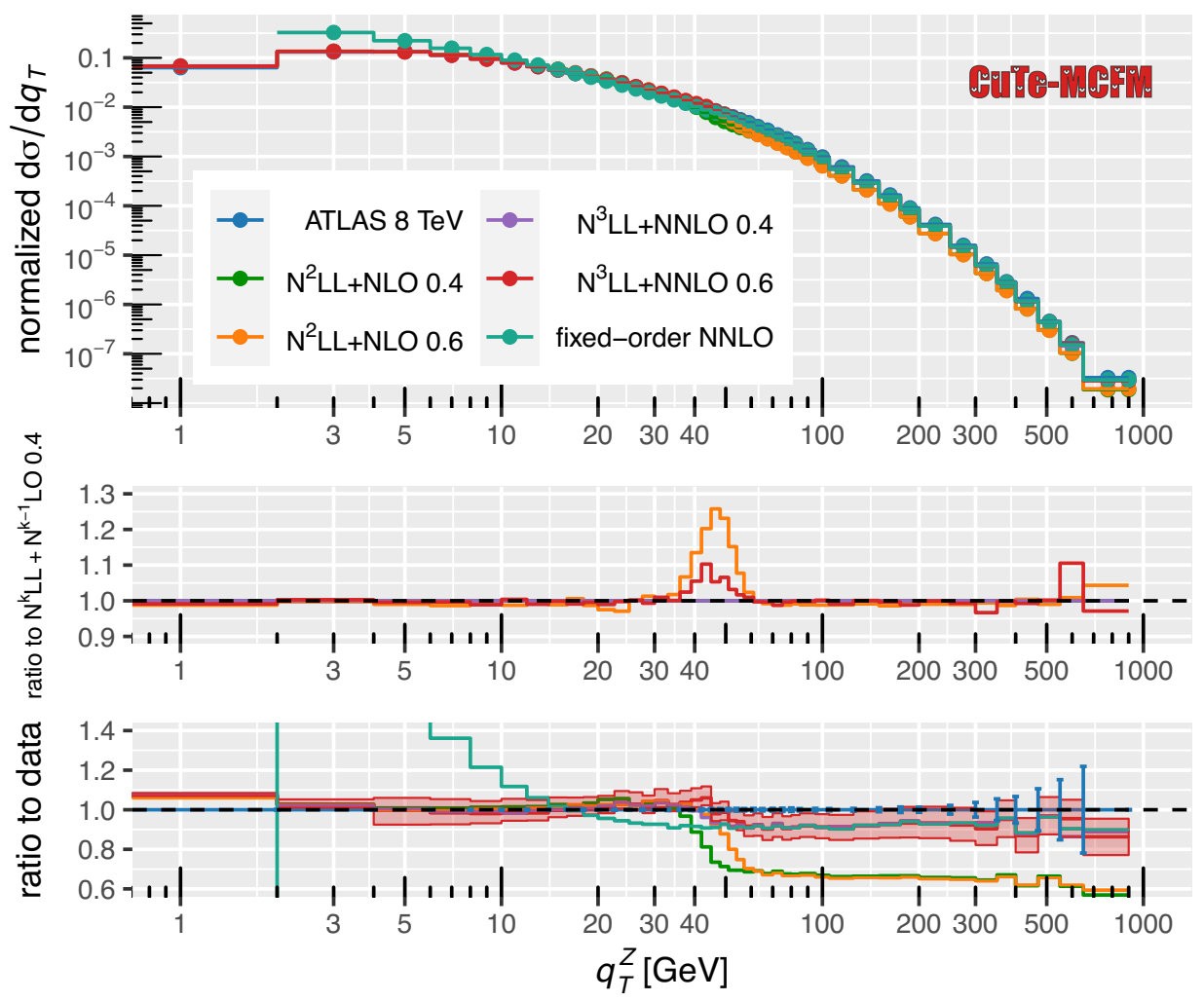

Figure 7. Predicted and measured normalized transverse-momentum distribution of the $Z$ boson with fiducial cuts as in the ATLAS study at $8 \mathrm{TeV}$ in ref. [99]. The middle panel shows the effect of varying the transition function, while the bottom panel shows the ratio to data with estimated scale uncertainties.

The first panel shows the normalized distribution of the data, the $\mathrm{N}^{2} \mathrm{LL}+\mathrm{NLO}$ and $\mathrm{N}^{3} \mathrm{LL}+\mathrm{NNLO}$ matched distributions with transition functions $x^{\max }=0.4,0.6$, and the fixedorder prediction. The fixed-order prediction is normalized by the $q_{T}$-integrated matched result for $q_{T}>2 \mathrm{GeV}$ with $x^{\max }=0.6$.

The middle panel shows the difference between using transition function parameters $x^{\max }=0.4$ and $x^{\max }=0.6$ for the matched results at order $\alpha_{s}$ and $\alpha_{s}^{2}$. At $\mathrm{N}^{2} \mathrm{LL}+\mathrm{NLO}$ the matching effects are at the order of $10-25 \%$ in the region of $40 \mathrm{GeV}$ to $60 \mathrm{GeV}$. A transition function that switches less rapidly than our choice would wash out the effects to a broader range, so this has to be considered when estimating the size of the matching effects. At $\mathrm{N}^{3} \mathrm{LL}+\mathrm{NNLO}$ the matching effects are much smaller, as one might expect, and below $10 \%$.

The bottom panel shows the ratio to the experimental data and includes a scaleuncertainty band for the $\mathrm{N}^{3} \mathrm{LL}+\mathrm{NNLO}$ prediction. Due to the normalization, the experimental uncertainties are at the sub-percent level for $q_{T}<150 \mathrm{GeV}$ and coincide with the dashed line on the displayed scale. Overall our highest-order prediction at $\mathrm{N}^{3} \mathrm{LL}+\mathrm{NNLO}$ describes the data very well up to large $q_{T}$ within five to ten percent uncertainties. At the largest shown $q_{T}$, relative QCD $\alpha_{s}^{2}$ effects would increase the cross section, but would have to be considered in addition to negative electroweak effects [109-111]. 


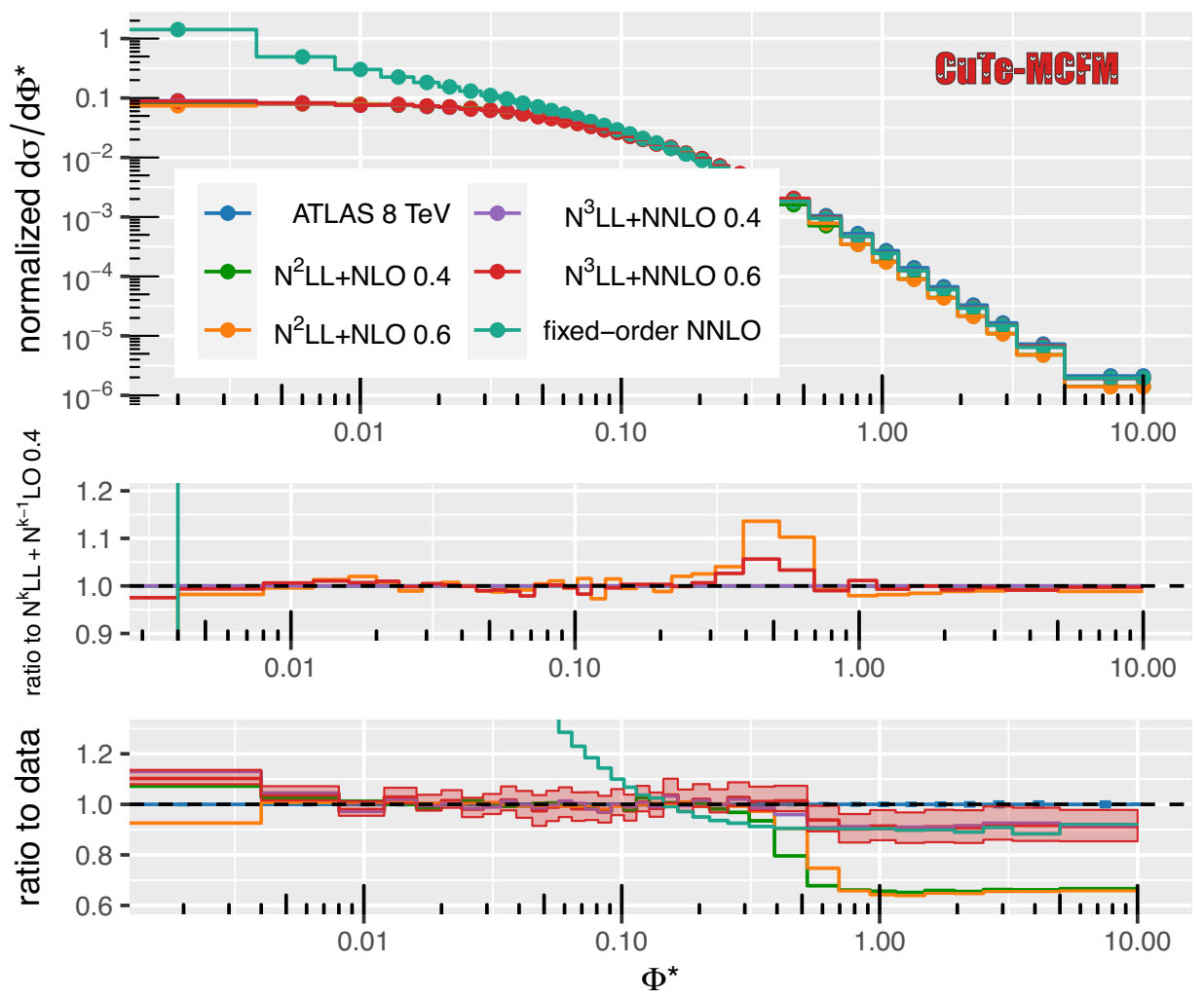

Figure 8. Predicted and measured $\phi^{*}$ distribution of the $Z$ boson with fiducial cuts as in the ATLAS study [99] at $8 \mathrm{TeV}$. The middle panel shows the effect of varying the transition function, while the bottom panel shows the ratio to data with estimated scale uncertainties.

The fixed-order result has scale uncertainties of about $\pm 10 \%$, which we do not display, to keep the plot easily readable. Since the fixed-order result agrees well with the resummed results within mutual uncertainties down to $10 \mathrm{GeV}$, the transition to fixed-order could be induced earlier than in range of $40 \mathrm{GeV}$ to $60 \mathrm{GeV}$ that we have used. Nevertheless, the resummation pushes the central prediction much closer to the data and results in agreement at the single-percent level. The presence of a large enough window for the matching is comforting and important to convince ourselves that we can combine the fixed-order and resummation results consistently and accurately.

The resummation formula in eq. (2.1) is fully differential in the electroweak momenta and can be used to also resum logarithms in other observables related to $q_{T}$. An example is the observable

$$
\phi^{*}=\tan \left(\frac{\pi-\Delta \phi}{2}\right) \sin \left(\theta^{*}\right),
$$

with $\cos \left(\theta^{*}\right)=\tanh \left(\frac{\Delta \eta}{2}\right)$, where $\Delta \eta$ is the pseudorapidity difference of the two charged leptons and $\Delta \phi$ the azimuthal angle between them. This quantity was introduced in refs. $[2,89]$ and has the advantage over $q_{T}$ that it can be extracted purely based on angular measurements on the leptons.

Since $\phi^{*} \propto q_{T}$ at small values, we also achieve full $\mathrm{N}^{3} \mathrm{LL}+\mathrm{NNLO}$ accuracy for the $\phi^{*}$ distribution as displayed in comparison with the measurement in figure 8 . We again exclude 


\begin{tabular}{|l|c|}
\hline Lepton cuts & $q_{T}^{l}>25 \mathrm{GeV},\left|\eta^{l}\right|<2.4$ \\
Separation cuts & $76.2 \mathrm{GeV}<m^{l^{+} l^{-}}<106.2 \mathrm{GeV},\left|y^{l^{+} l^{-}}\right|<2.4$ \\
\hline
\end{tabular}

Table 2. Fiducial for $Z \rightarrow l^{+} l^{-}$at $\sqrt{s}=13 \mathrm{TeV}$, see ref. [100].

the region corresponding to small $q_{T}$ and normalize to the integrated result for $\phi^{*}>0.004$. The conclusions reached for the $q_{T}$ distribution discussed earlier apply also here, both qualitatively and quantitatively. The effects from the matching are overall smaller than $5 \%$ for the $\mathrm{N}^{3} \mathrm{LL}+\mathrm{NNLO}$ prediction, as can be seen from the second panel. The third panel shows the ratio to the experimental data and demonstrates a fantastic agreement with our prediction within scale uncertainties. Between $\phi^{*}=0.1$ and 0.5 fixed-order prediction and resummed prediction have a large window of agreement that indicates a well-behaved perturbative expansion.

\subsubsection{CMS measurements at $13 \mathrm{TeV}$}

As a second example, we directly compare with $13 \mathrm{TeV}$ cross-section data from CMS [100] in figure 9, without normalizing the results. The applied cuts are presented in table 2. We again choose the dynamic hard scale as $\mu_{h}=\sqrt{Q^{2}+q_{T}^{2}}$ and use the NNPDF31_nnlo_as_0118 PDF set.

Overall the conclusions are similar to our findings for the normalized predictions shown at $8 \mathrm{TeV}$ before. Up to $40 \mathrm{GeV}$ the resummed result (matched with small matching corrections) agrees at the percent level with data. Only in the first bins the small scale uncertainties and a deviation of up to $10 \%$ hint towards non-perturbative effects. Note that the small scale bands do not cover the true uncertainty, see section 2 .

While non-perturbative transverse-momentum effects would be captured by fitting transverse-momentum dependent PDFs, also the standard PDFs encode non-perturbative physics. To study the associated uncertainties, we computed the PDF uncertainties for multiple PDF sets and show the result in figure 10. On a technical level, this demonstrates the efficient and accurate evaluation of PDF uncertainties in MCFM-9 and consequently also in our setup CuTe-MCFM.

The minimum scale value of $Q^{\min }=4.47 \mathrm{GeV}$ for the ABMP16 PDF set causes the predictions to break down when our scale is set to a value lower than this. To fix this issue, one could, in principle, perform a DGLAP evolution below this scale, or enforce a minimum scale of $Q^{\text {min }}$ in our resummation code. Instead we deliberately show the result with the default settings of LHAPDF and our default minimum safety scale of $2 \mathrm{GeV}$.

The other PDFs broadly predict uncertainties above $10 \%$ below $q_{T}=2 \mathrm{GeV}$, and CT18 even predicts uncertainties of more than $20 \%$. The $10 \%$ difference between our prediction and data in the first bin of figure 9 is therefore well within even just PDF uncertainties.

\subsection{Fiducial $W$ production as measured by CMS at $8 \mathrm{TeV}$}

While the transverse-momentum distribution of the charged lepton in $W$ production enters many precision analyses, the fully reconstructed $W$ boson transverse-momentum distribution has also been presented by CMS at $8 \mathrm{TeV}$ [101] and at $7 \mathrm{TeV}$ by ATLAS [112]. 


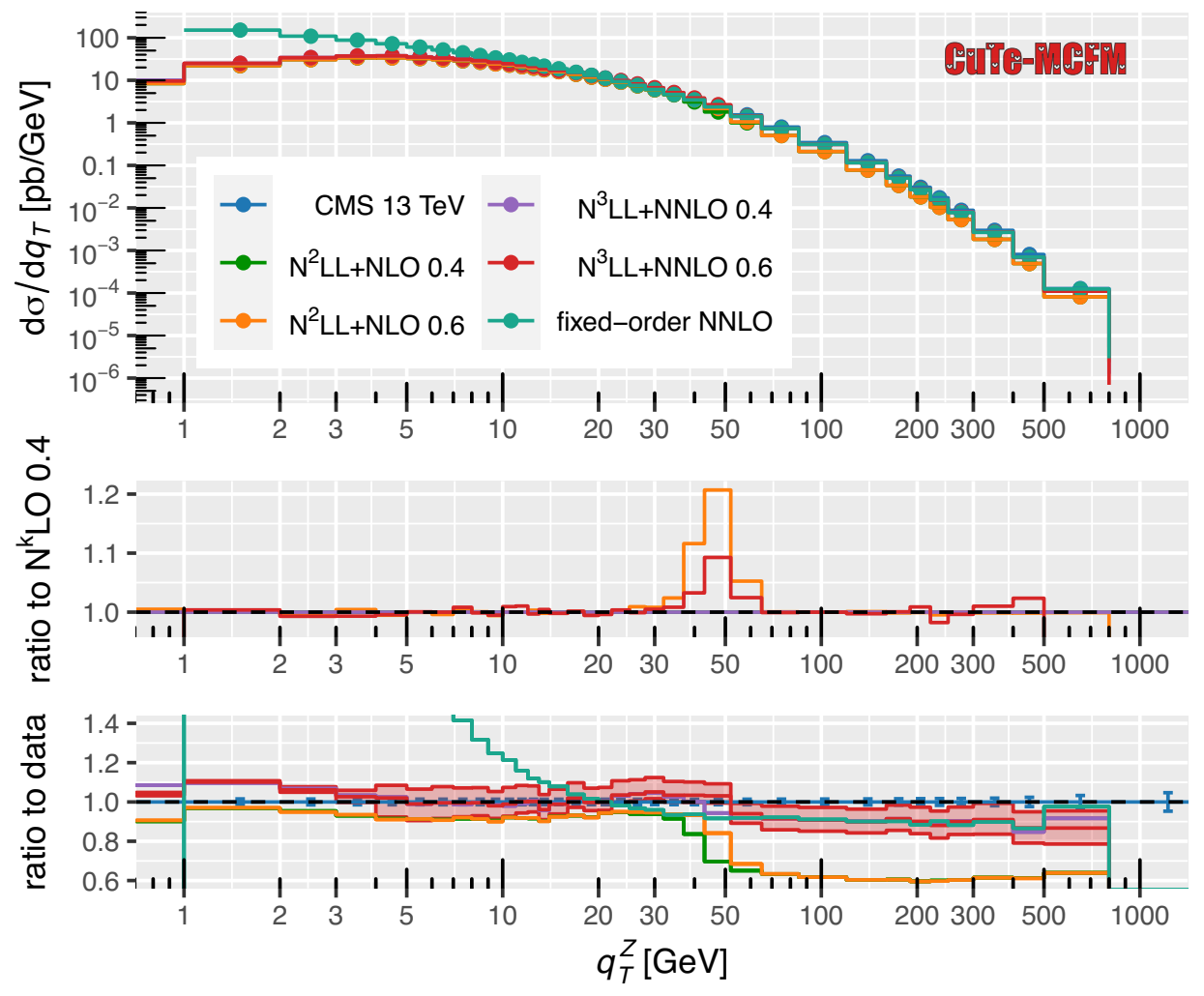

Figure 9. Predicted and measured transverse-momentum distribution of the $Z$ boson with fiducial cuts as in the CMS study [100] at $13 \mathrm{TeV}$. The middle panel shows the effect of varying the transition function, while the bottom panel shows the ratio to data with estimated scale uncertainties.

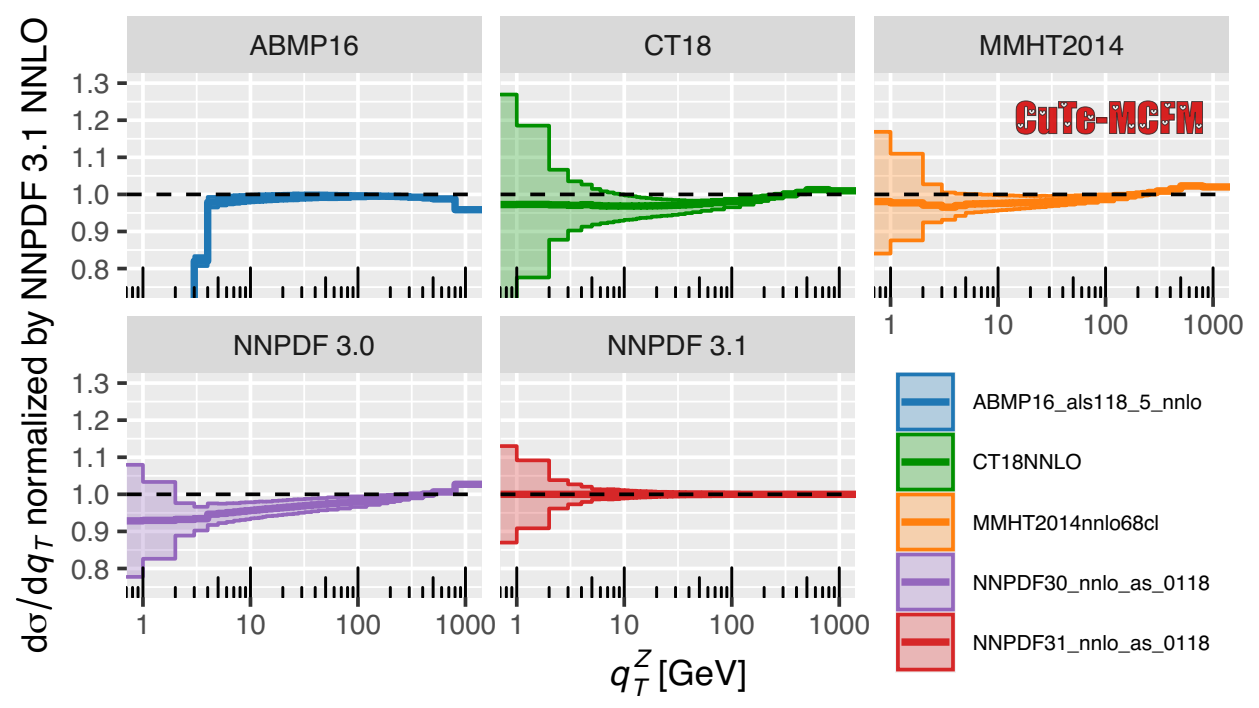

Figure 10. Z-boson transverse-momentum distribution including PDF uncertainties for various PDF sets normalized to the prediction with the NNPDF3.1 NNLO central value. See figure 9. 

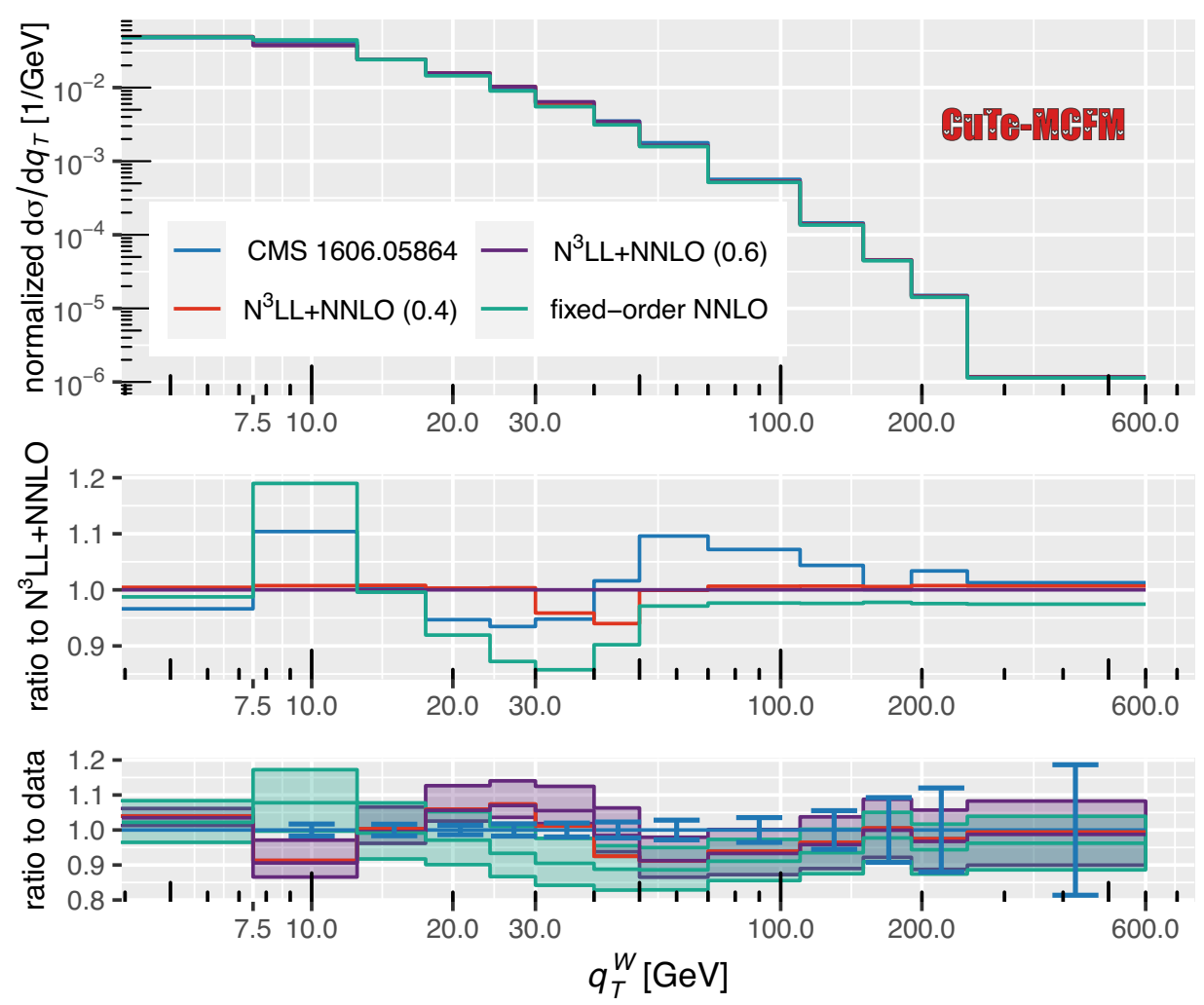

Figure 11. Comparison to normalized $W$ transverse-momentum data from CMS at $8 \mathrm{TeV}$ with predictions at $\mathrm{N}^{3} \mathrm{LL}+\mathrm{NNLO}$ including uncertainties associated with scale variation.

Here we compare with the normalized $8 \mathrm{TeV}$ CMS data, where both $W^{-}$and $W^{+}$ channels are added. The only applied cuts are a minimum $q_{T}$ of $25 \mathrm{GeV}$ and a maximum absolute pseudorapidity of 2.5 on the electron/positron. We furthermore choose a central hard scale of $\mu_{h}=\sqrt{Q^{2}+q_{T}^{2}}$ and the PDF set CT18NnLO. As for $Z$ production, the resummed logarithms describe the full result at an impressive level with matching corrections that stay just at the few percent-level for $q_{T}^{W} \lesssim 40 \mathrm{GeV}$, reaching about 30 percent for $60 \mathrm{GeV}$ (not shown).

We show our matched results in figure 11, where the first panel shows the normalized distribution. The second panel shows the ratio to our matched result with $x^{\max }=0.6$ to guide the eye on the difference between the two transition functions with $x^{\text {max }}=0.4,0.6$. The resulting difference between our two choices of $x^{\max }$ can be seen around $30 \mathrm{GeV}$ to $50 \mathrm{GeV}$ where the two distributions differ by about five percent. This estimates the size and position of the matching uncertainty.

The third panel shows the ratio to the CMS data and now includes a scale-variation band for the fixed-order prediction, the fully matched prediction, as well as uncertainties for the data. While we would have expected to find very good agreement in the region where the matching corrections are small $(\lesssim 40 \mathrm{GeV})$, the overall agreement to the data is not better than the fixed-order prediction, but overall we find agreement within scale uncertainties. 


\begin{tabular}{|l|l|}
\hline Photon cuts & $q_{T}^{\gamma}>40 \mathrm{GeV}, 30 \mathrm{GeV},\left|\eta_{\gamma}\right|<2.5$ \\
Smooth-cone photon isolation & $E_{T}^{\gamma, \max }=10 \mathrm{GeV}, R=0.3, n=1$ \\
\hline
\end{tabular}

Table 3. Fiducial cuts for $H \rightarrow \gamma \gamma$ at $\sqrt{s}=13 \mathrm{TeV}$.

The most striking difference of $10 \%$ between central prediction and data is in the second bin from $7.5 \mathrm{GeV}$ to $12.5 \mathrm{GeV}$. This deviation can also be observed in the CMS publication [101] when the data is compared with $\mathrm{N}^{2} \mathrm{LL}$ resummed predictions. This difference and the overall shape of the data for $q_{T} \lesssim 40 \mathrm{GeV}$ is perhaps indicative a systematic issue with the experimental analysis at low $q_{T}$, but it is not possible to make a definite statement since the results are compatible within mutual uncertainties.

\subsection{Fiducial $H \rightarrow \gamma \gamma$ benchmark}

The Higgs transverse-momentum distribution has been measured by CMS and ATLAS at $8 \mathrm{TeV}$ and $13 \mathrm{TeV}$ in various production and decay channels. But even after a combination the overall uncertainties are at the order of $40 \%$ or worse [113].

For a precise study and prediction, one should at the least take into account top-quarkmass effects and consider the resummation of $\pi^{2}$ terms [114, 115]. Further contributions like bottom-quark-mass effects have also been studied at low $q_{T}[116]$ and become relevant at the percent level for the resummation. Within the MCFM framework top-quark-mass effects have been included throughout NLO accuracy for $q_{T} \gg m_{t}$ and $q_{T} \ll m_{t}[117-119]$ and NNLO corrections have been presented in the EFT for large $q_{T}[120,121]$. Including these mass effects goes beyond the scope of our paper and we only show results in the heavytop-quark limit. For now we present results without comparison to data, but include a set of cuts as used in experiments, see table 3 .

In figure 12 we show matched results for the Higgs transverse-momentum distribution with fiducial cuts as in table 3 using the MMHT2014nnlo68cl PDF set and a central hard scale of $\mu_{h}=\sqrt{m_{H}^{2}+q_{T}^{2}}$. The second panel shows the effect of the matching to fixed order by switching between the transition function parameters $x^{\max }=0.4$ and 0.6. Matching effects are about $10 \%$ in the region of $50 \mathrm{GeV}$ to $80 \mathrm{GeV}$ and the resummation stabilizes the fixedorder predictions below such values. At small values of $q_{T} \lesssim 2 \mathrm{GeV}$ the cancellations within the matching corrections are numerically difficult and reflect in the larger fluctuations.

The bottom two panels show the effect of PDF uncertainties relative to our central prediction. At $q_{T} \lesssim 5 \mathrm{GeV}$ uncertainties of more than $10 \%$ have to be added to the already sizeable scale uncertainties. While these uncertainties add up to just give an order of magnitude prediction, the uncertainties from $\alpha_{s}$ itself, to which gluon fusion Higgs production is highly sensitive, are not even included yet. The road towards precision Higgs transverse-momentum measurements and predictions is therefore a long one, but using the normalized distribution would mitigate some of these additional uncertainties.

\subsection{Fiducial $\gamma \gamma$ production}

In this section we present results for fiducial diphoton production. The fixed-order NNLO result in MCFM is based on ref. [93]. NNLO results have also been presented in ref. [122], 

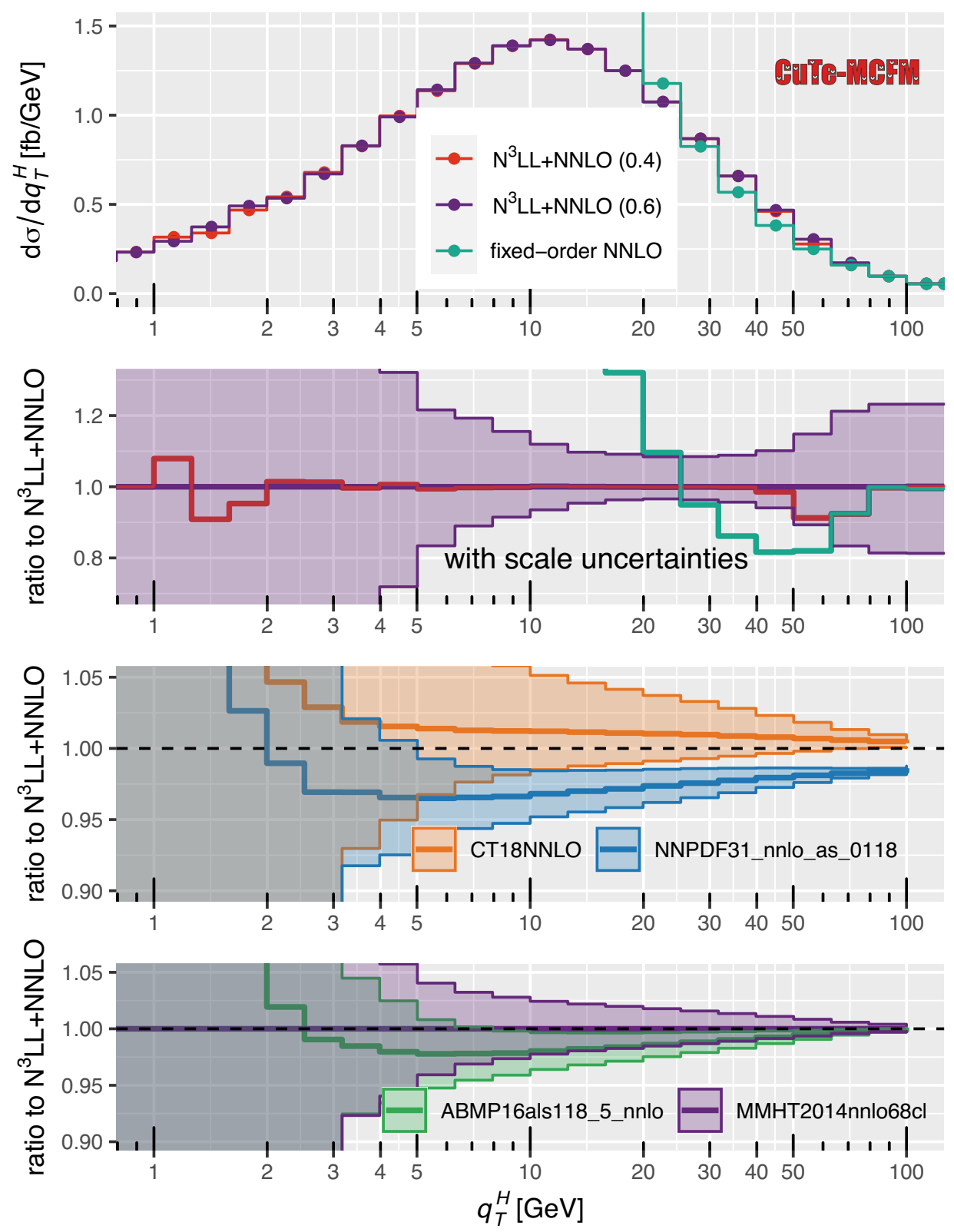

Figure 12. Higgs transverse-momentum distribution with fiducial cuts as in table 3 . The second panel shows the effect of a different transition function and scale uncertainties, while the bottom panels show PDF uncertainties for different sets.

which were subsequently interfaced with $\mathrm{N}^{2} \mathrm{LL} q_{T}$ resummation [123]. Resummation at $\mathrm{N}^{2}$ LL has also been presented in refs. [42, 124-126] matched to NLO.

Perturbative NNLO corrections in diphoton production are large and increase NLO results by 50-75\%, depending on cuts. The gluon-gluon channel, which first appears at NNLO through a quark-box diagram, also constitutes a noticeable part of these corrections. Therefore, only at $\mathrm{N}^{3} \mathrm{LO}$ one has control at the NLO level over all partonic channels. Twoloop NLO corrections for the gluon-gluon channel have been calculated in refs. [127, 128]. 


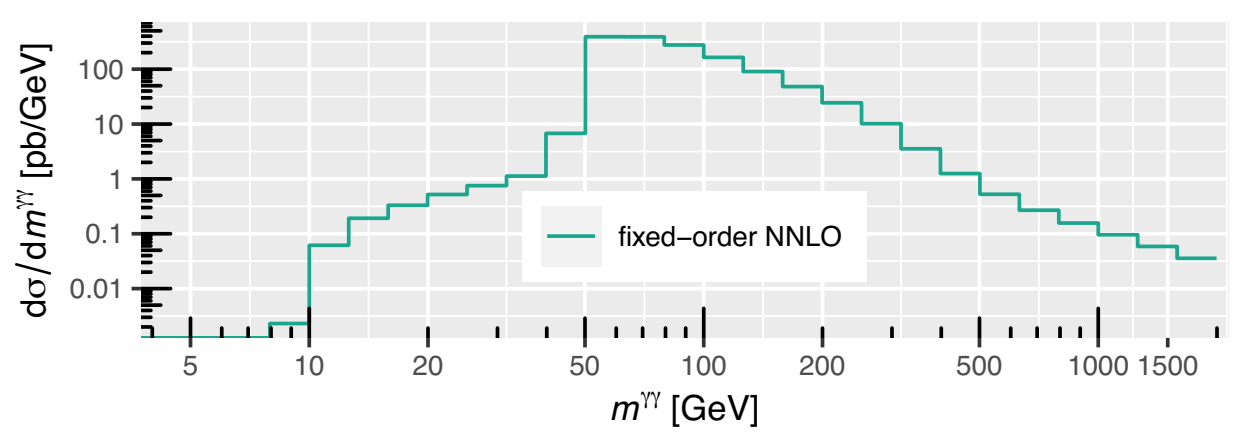

Figure 13. Diphoton invariant mass distribution at NNLO with $q_{T}^{\gamma, 1}>25 \mathrm{GeV}, q_{T}^{\gamma, 2}>22 \mathrm{GeV}$ and further cuts specified in table 4 .

\begin{tabular}{|l|c|}
\hline Photon cuts & $q_{T}^{\gamma, 1}>25 \mathrm{GeV}, q_{T}^{\gamma, 2}>22 \mathrm{GeV}$ \\
& $\left|\eta_{\gamma}\right|<2.37,1.56<\left|\eta_{\gamma}\right|<1.37$ \\
Photon separation & $\Delta R(\gamma, \gamma)>0.4$, \\
Smooth-cone photon isolation & $E_{T}^{\gamma, \max }=4 \mathrm{GeV}, n=1, R=0.4$ \\
\hline
\end{tabular}

Table 4. Fiducial in diphoton production at $\sqrt{s}=7 \mathrm{TeV}$, see ref. [131].

These have later been implemented in MCFM together with the NNLO corrections to the $q \bar{q}$ channel [93] and constitute a part of the $\mathrm{N}^{3} \mathrm{LO}$ corrections. We also discuss these and show the effect of including them in the following.

A common requirement for diphoton production is that both photons have specific minimum transverse momenta, $q_{T, \text { min }}^{\gamma, 1}$ (harder) and $q_{T, \text { min }}^{\gamma, 2}$ (softer), where $q_{T, \text { min }}^{\gamma, 1}>q_{T, \text { min }}^{\gamma, 2}$. For transverse momenta larger than $q_{T, \text { min }}^{\gamma, 1}+q_{T, \text { min }}^{\gamma, 2}$ both photons can be aligned in the same direction and recoil against hadronic radiation. This threshold can be seen for example in the diphoton invariant mass distribution shown in figure 13, which strongly peaks above $\sim$ $q_{T, \min }^{\gamma, 1}+q_{T, \min }^{\gamma, 2}=47 \mathrm{GeV}$. (As a side remark, we note that the cusp in this distribution could be removed by an appropriate soft gluon resummation $[129,130]$.$) Transverse-momentum$ resummation is no longer valid for $q_{T}$ values above this threshold and becomes numerically unstable, so that one wants to fully switch to the fixed-order prediction above this threshold. To not introduce a discontinuity, the transition function has to be chosen to give negligible contributions from $q_{T}>q_{T, \text { min }}^{\gamma, 1}+q_{T, \text { min }}^{\gamma, 2}$. Of course, from the resummation point of view, it would be best to impose a lower cut on the invariant mass of the two photons, which would avoid these problems.

\subsubsection{ATLAS measurements at $7 \mathrm{TeV}$}

We first compare with the $7 \mathrm{TeV}$ ATLAS diphoton measurement [131]. The fiducial phase space is defined by the cuts in table 4. Our results here are presented using the PDF set MSTW2008nnlo68cl [106] and a central hard scale of $\mu_{h}=m_{\gamma \gamma}$, following the choice in the previous study at $\mathrm{N}^{2} \mathrm{LL}$ [123].

Our detailed discussion in section 2 (page 11), showed that for smooth-cone isolation (and $n=1$ ) linear power corrections are present. To account for this, we could modify our 

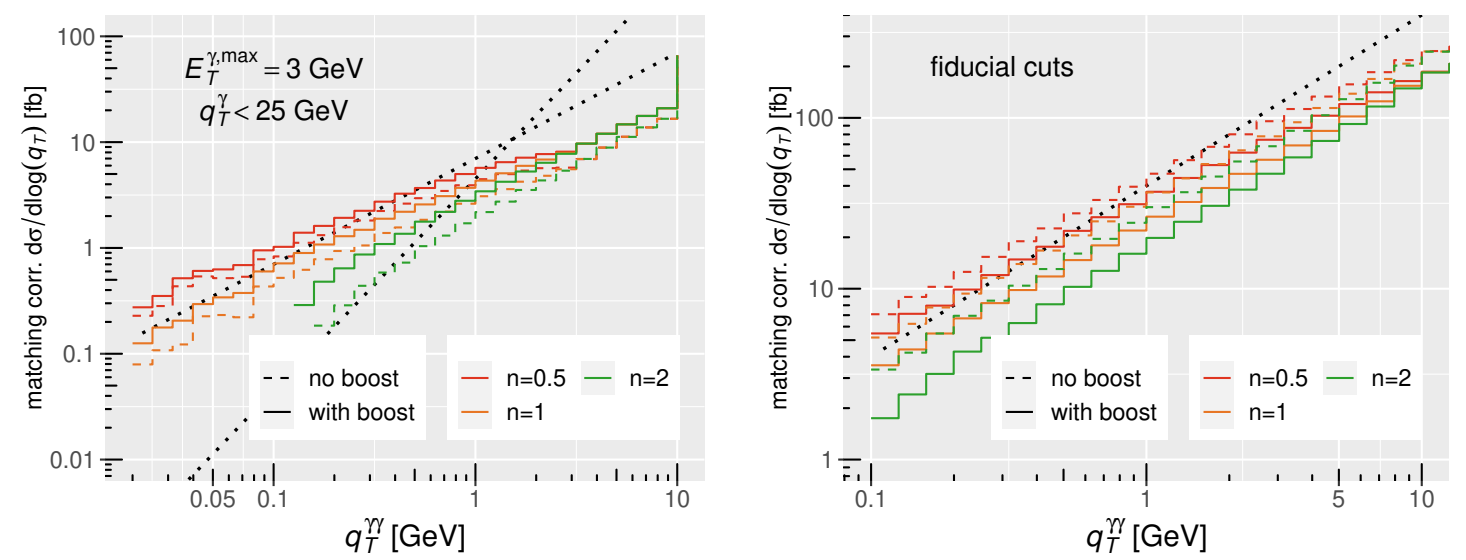

Figure 14. Matching corrections at $\mathrm{N}^{2} \mathrm{LL}$ for diphoton production with photon $q_{T}$ and photon isolation cuts only (left) and all fiducial cuts in table 4 (right). The different colors correspond to different values of $n$. The solid lines are the power corrections after accounting for recoil, the dashed lines are without recoil. The black dotted lines indicate first and second order scaling in $q_{T}$ to guide the eye.

transition function to be a function of $q_{T} / Q$ without spoiling power corrections. Instead, we keep it as a function of $q_{T}^{2} / Q^{2}$, but choose a sufficiently smaller parameter $x^{\text {max }}$ determining the transition region. We find that $x^{\max }=0.1,0.2$ are sufficiently small such that we can can fully switch to the fixed-order result above $47 \mathrm{GeV}$, but can also study the effect of the transition to the fixed-order result.

In figure 2 in section 2 , we have discussed the asymptotic scaling of the power corrections with the isolation parameter $n$ and have shown numerical results for the partonic $q \bar{q}$ channel. We now show the sum of all partonic channels, with and without fiducial cuts, in figure 14. The behavior is qualitatively different than in the $q \bar{q}$ channel shown earlier in figure 2: at least in the $q_{T}$ range we consider, the power corrections are approximately linear, and relatively insensitive to the choice of $n$. Furthermore, even for $n=2$, where power corrections scale like $\sqrt{q_{T}}$ in the $q \bar{q}$ channel, they are somewhat more suppressed when considering all partonic channels.

The reason for the different behavior is that the power corrections associated with a gluon radiated inside the cone, as present in the $q \bar{q}$ channel, are suppressed by $R^{2}$, in contrast to the ones associated with the fragmentation correction, see eqs. (2.16) and (2.17). These two contributions also enter with different signs, so that for non-asymptotic values of $q_{T}$ cancellation effects occur. Eventually, for $n=2$ and sufficiently small $q_{T}$, the $R^{2}$ suppression is overcome and the asymptotic behavior should set in and one would expect to observe $\sqrt{q_{T}}$ scaling again.

As discussed in section 2, the presence of linear power corrections implies that the matching corrections no longer go to zero in $d \sigma / d q_{T}$. We show these corrections at $\mathrm{N}^{2} \mathrm{LL}$ in figure 15 relative to the naively matched result. Here, we are interested in larger $q_{T}$ values of practical relevance and not in the asymptotic behavior. We include different choices of the photon isolation parameters $n$ and $R$. The purple curve with $n=1$ and $R=0.4$ corresponds to the default fiducial cuts in table 4 . 


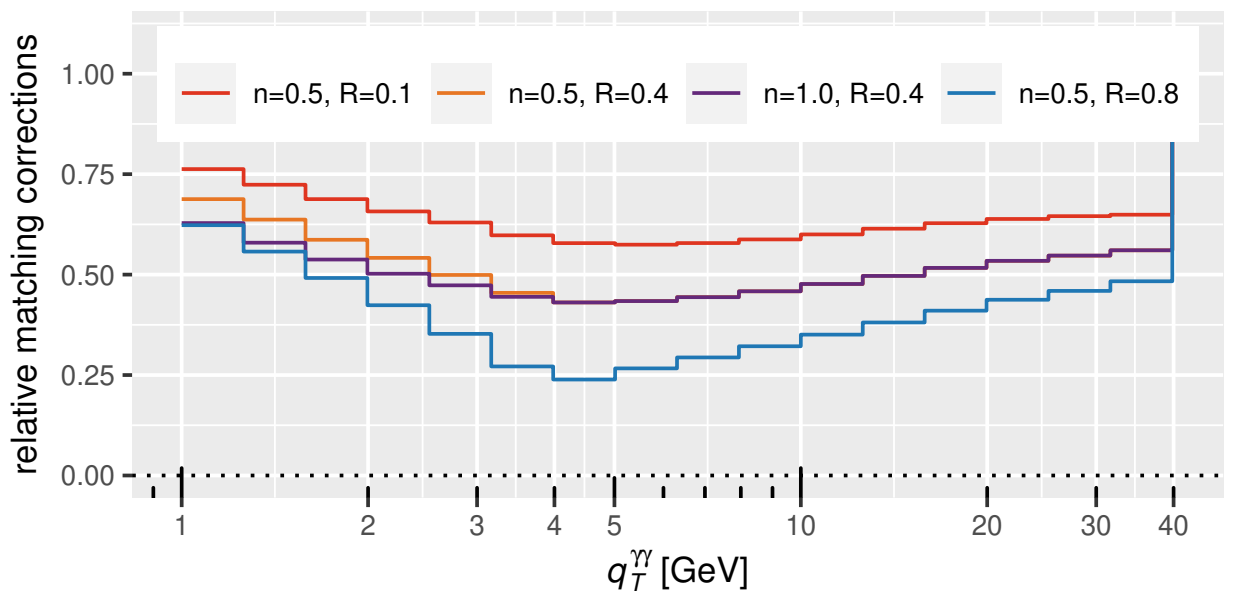

Figure 15. Diphoton maching corrections at $\mathrm{N}^{2} \mathrm{LL}$ relative to naively matched results with cuts as in table 4 , but for different choices of the photon isolation parameters $n$ and $R$.

Already at small $q_{T}$ the purple matching corrections start at about $60 \%$ and never dip below $40 \%$. Since the matching corrections go to a constant for $q_{T} \rightarrow 0$, a characteristic of the linear power corrections, and the resummed result approaches zero, the relative matching corrections eventually approach $100 \%$ for $q_{T} \rightarrow 0$. Note that this does not signify an issue with our implementation of the $q_{T}$ resummation. We have checked that the fixed-order expansion of the resummed result cancels with the fixed-order result down to the sub per-mille level for very small $q_{T}$. The correct cancellation can indeed be observed as the (linearly) vanishing matching corrections in figure 14 .

With figure 15 we can now also discuss the effect of cancellations between photonisolation power corrections associated with fragmentation and associated with gluon emission for moderate values of $q_{T}$. These contributions enter with opposite signs, which has a peculiar effect on the $R$-dependence: naively one might expect smaller power corrections with a smaller $R$, since the gluon radiation power corrections scale like $R^{2}$. But these negative power corrections have to be added to the positive and larger power corrections from the fragmentation contribution. One therefore observes a cancellation and overall smaller matching corrections for larger $R$.

At $\mathrm{N}^{3} \mathrm{LL}$ the observed large matching corrections do not change qualitatively, as shown in figure 16. The matching corrections now start just below $50 \%$ around $q_{T}=2 \mathrm{GeV}$ and reach $75 \%$ just before the resummation validity threshold of $47 \mathrm{GeV}$.

Since we do not include matching corrections below $1 \mathrm{GeV}$ for numerical stability, we neglect sizeable effects below this value. Taken at face value, the matching would amount to a $50 \%$ effect. A resummation of power-suppressed terms would likely suppress the matching corrections, but their true size is difficult to estimate. The situation is different from processes where the matching corrections are quadratic and such a safety cutoff of $1 \mathrm{GeV}$ leads to small effects. While such a hard cutoff is relatively unproblematic for the $q_{T}$ distribution itself, it is more difficult for other observables that benefit from resummation, like $\phi^{*}$ or the azimuthal angle difference between the photons $\Delta \phi$. The cutoff may affect a 


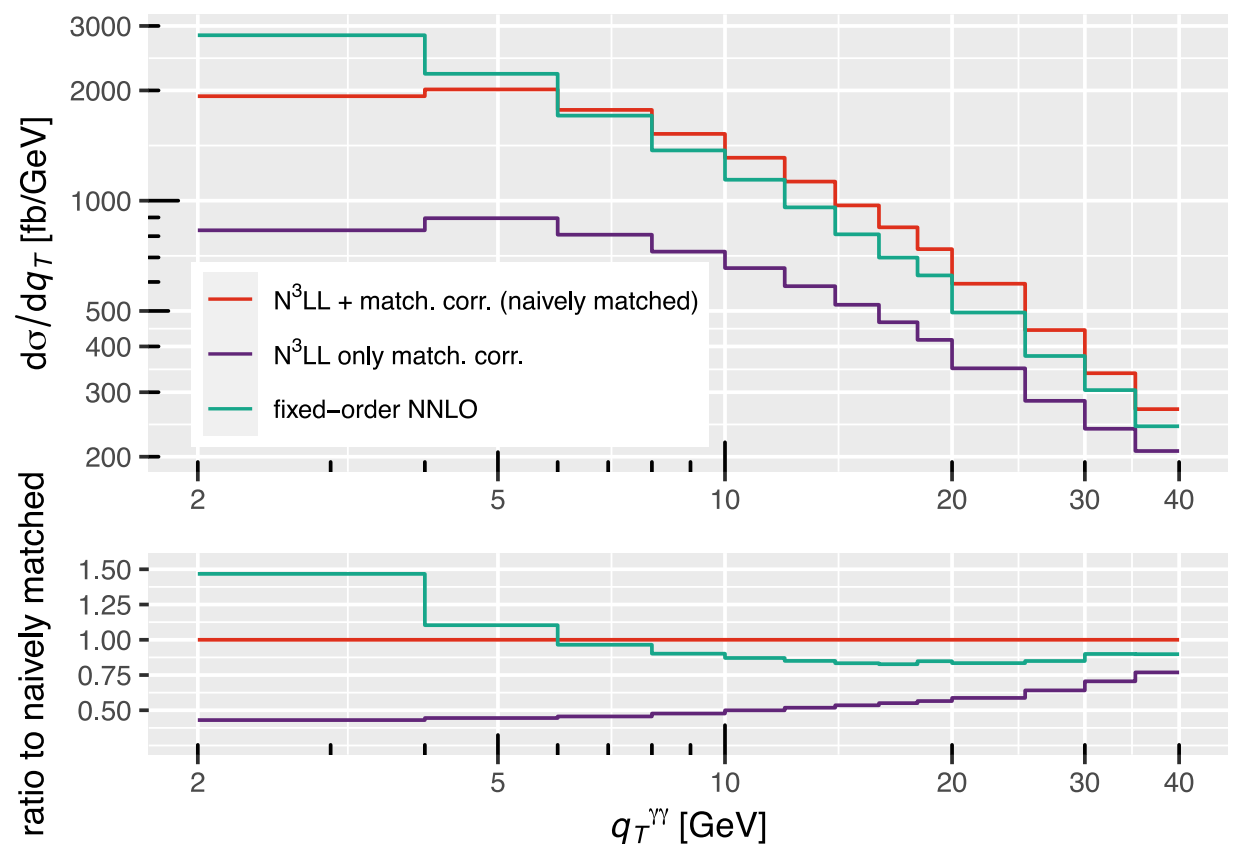

Figure 16. Comparison of the naively matched result at $\mathrm{N}^{3} \mathrm{LL}+\mathrm{NNLO}$, the matching corrections and the fixed-order result for diphoton production with the cuts used by ATLAS [131], see table 4. The second panel displays the ratio to the naively matched result to demonstrate the large matching corrections even at small $q_{T}$.

broader spectrum for such observables, and not just one bin. Fortunately, at least for $\phi^{*}$ only low values are affected since $\phi^{*} \leq q_{T} / Q$. To do better than this somewhat arbitrary cutoff prescription, we would need to determine and include the Sudakov suppression factor for the power-suppressed terms.

Overall, since the first few $\mathrm{GeV}$ in $q_{T}$ are likely to receive non-perturbative effects (see for example the parametrization of non-perturbative effects in ref. [123]), we can disregard the first experimental bin (from $0 \mathrm{GeV}$ to $2 \mathrm{GeV}$ ) for a meaningful comparison in this study. For observables like $\phi^{*}$ or $\Delta \phi$ similar arguments regarding non-perturbative corrections hold so that predictions for the regions corresponding to values $q_{T} \sim 0$ need to be studied very carefully.

Having analyzed the matching corrections in detail, we now choose the transition function with $x^{\max }=0.1,0.2$, as indicated earlier, and present our matched $\mathrm{N}^{3} \mathrm{LL}+\mathrm{NNLO}$ results in figure 17. The upper panel in this figure shows the absolute $\mathrm{N}^{3} \mathrm{LL}+\mathrm{NNLO}$ matched distribution with the two choices of $x^{\text {max }}$, the NNLO fixed-order result, as well as the measurement.

The second panel shows corresponding ratios to the matched $\mathrm{N}^{3} \mathrm{LL}+\mathrm{NNLO}$ result with $x^{\max }=0.1$. The agreement with data in the region of up to $30 \mathrm{GeV}$ is clearly improved, with resummation effects of up to $13 \%$ around $15 \mathrm{GeV}$. Beyond $\sim 45 \mathrm{GeV}$ practically only the fixed-order result contributes. The filled regions denote the experimental uncertainties for the ATLAS data and uncertainties from scale variation for the matched result, respectively. 

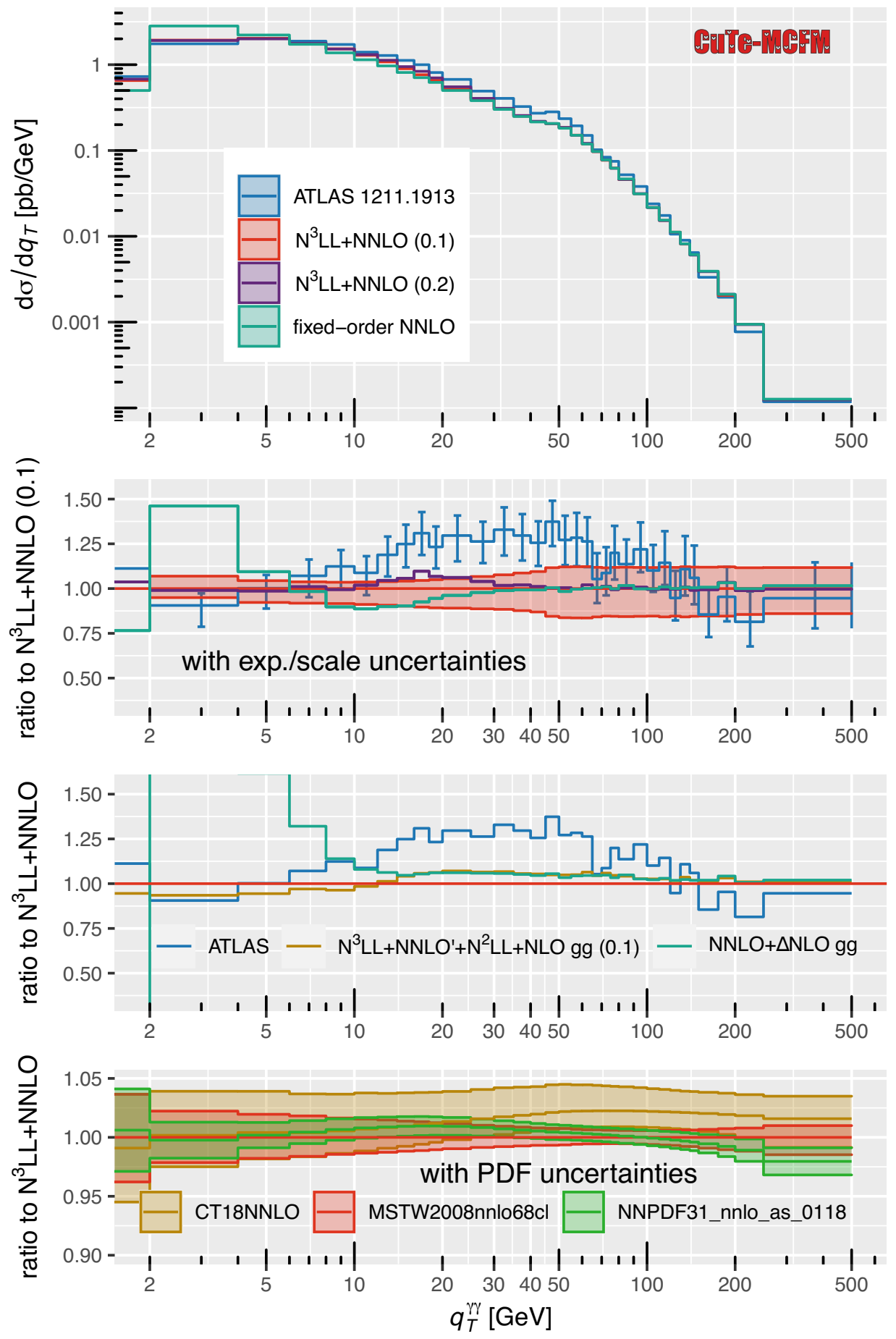

Figure 17. Comparison of $7 \mathrm{TeV}$ ATLAS diphoton results with predictions in various approximations including uncertainties associated with scale variation, PDFs and NLO contributions to the gluon-gluon channel; see text for details. The labels 0.1 and 0.2 in the plots refer to the value of $x^{\max }$. 


\begin{tabular}{|c|c|}
\hline Photon cuts & $\begin{array}{c}q_{T}^{\gamma, 1}>40 \mathrm{GeV}, q_{T}^{\gamma, 2}>30 \mathrm{GeV} \\
\left|\eta_{\gamma}\right|<2.37,1.56<\left|\eta_{\gamma}\right|<1.37\end{array}$ \\
\hline Photon separation & $\Delta R(\gamma, \gamma)>0.4$ \\
\hline Smooth-cone photon isolation & $E_{T}^{\gamma, \max }=11 \mathrm{GeV}, n=1, R=0.4$ \\
\hline
\end{tabular}

Table 5. Fiducial cuts in diphoton production at $\sqrt{s}=8 \mathrm{TeV}$, see ref. [132].

For brevity we do not include uncertainties for the fixed-order prediction, which agree above $q_{T}^{\gamma \gamma} \gtrsim 50 \mathrm{GeV}$ with the matched result and are similarly sized below.

Including the gluon-gluon channel at NLO has been found to be an important contribution at fixed-order perturbation theory [93]. Therefore, in the third panel we additionally display the ratios where the gg-channel is included at NLO and $\mathrm{N}^{2} \mathrm{LL}+\mathrm{NLO}$. The fixed-order result is obtained by adding the NLO gg-channel gg $\rightarrow \gamma \gamma$ g to the NNLO fixed-order result without the gg-channel. The resummed result is obtained by matching at $\mathrm{N}^{3} \mathrm{LL}$ with the NNLO result without gg-channel, and adding the $\mathrm{N}^{2} \mathrm{LL}+\mathrm{NLO}$ resummed gg-channel.

While the fixed-order result indeed receives sizeable corrections from the $\mathrm{gg} \rightarrow \gamma \gamma \mathrm{g}$ channel in the region up to $\sim 50 \mathrm{GeV}$, the corrections from the matched result change little compared to the overall uncertainties and agreement with data. In fact, the improved fixed-order result (cyan) and improved matched result (yellow) agree above $15 \mathrm{GeV}$. This indicates a significant stabilization of the perturbative series through the NLO corrections in the $g g$-channel.

Finally, the fourth panel displays the comparatively small PDF uncertainties at the level of a few percent (with a fixed value of $\alpha_{s}$ ).

\subsubsection{ATLAS measurements at $8 \mathrm{TeV}$}

Next, we compare against the most recent diphoton ATLAS measurement at $8 \mathrm{TeV}$ [132] which also considers $\phi^{*}$ for this process, as defined in eq. (3.1), but using the photon instead of the lepton directions. For this study we impose the cuts listed in table 5. We now choose a central hard scale of $\mu_{h}=\sqrt{m_{\gamma \gamma}^{2}+\left(q_{T}^{\gamma \gamma}\right)^{2}}$ and NNPDF30_nnlo_as_0118 as our default PDF set. Given the minimum transverse momenta of the photons we ensured that our transition function fully switches to the fixed-order result beyond $70 \mathrm{GeV}$.

Our results for the observables $q_{T}, \phi^{*}$ and $\Delta \phi^{\gamma \gamma}$, the azimuthal-angle difference between the two photons, are shown in figures 18, 19 and 20, respectively. For each figure the first panel shows the absolute distribution, the second panel the results in ratio to the matched result with $x^{\max }=0.1$, and the third panel the results in ratio to the experimental data. The uncertainties associated with the matching to fixed order can be read off from the second panel and are about $5-10 \%$ in the region of $15 \mathrm{GeV}$ to $40 \mathrm{GeV}$ in the $q_{T}$ distribution and an equivalent amount in the $\phi^{*}$ distribution around 0.1-0.4. For $\Delta \phi^{\gamma \gamma}$ they correspond to a region of $\sim 2.4-2.8$.

The resummation of course stabilizes predictions for all observables in the region below $10 \mathrm{GeV}$. Beyond that the resummed result improves the agreement with data up to $15 \%$. For the $q_{T}$ distribution we find agreement of predictions with data within uncertainties be- 

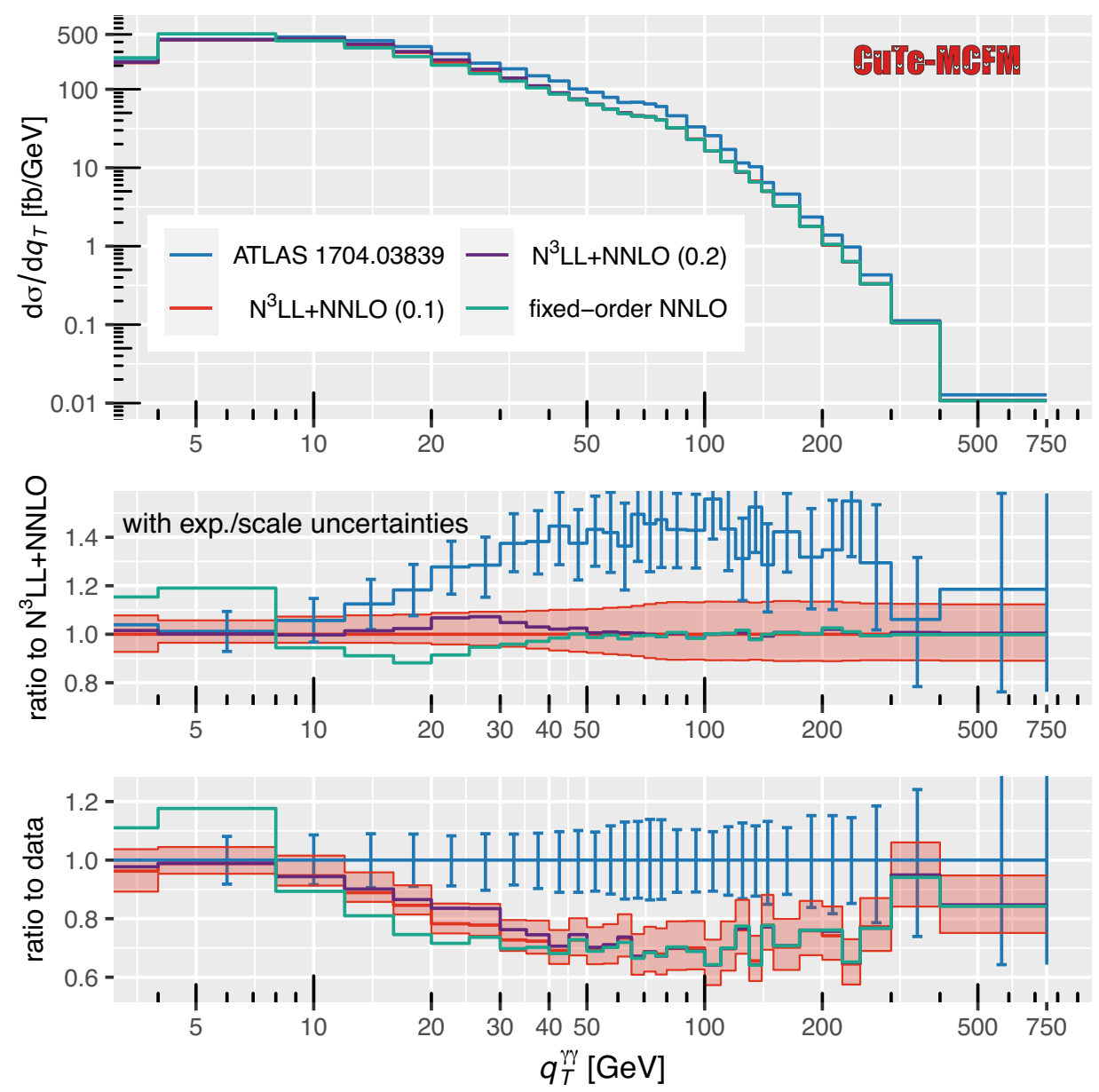

Figure 18. Comparison of the ATLAS measurement of the diphoton transverse-momentum distribution at $8 \mathrm{TeV}$ with predictions at $\mathrm{N}^{3} \mathrm{LL}+\mathrm{NNLO}$ including uncertainties associated with scale variation. The labels 0.1 and 0.2 in the plots refer to the value of $x^{\max }$.

low $20 \mathrm{GeV}$. Unfortunately at large $q_{T}$ the fixed-order NLO predictions are still insufficient to fully describe the data. We do not show distributions at the lower precision $\mathrm{N}^{2} \mathrm{LL}+\mathrm{NLO}$ since these significantly underestimate the size of the cross sections. We therefore expect that $\alpha_{s}^{3}$ corrections are necessary to achieve agreement with data in the region of large $q_{T}$. Similar conclusions hold for the $\phi^{*}$ and $\Delta \phi^{\gamma \gamma}$ distributions.

While our $1 \mathrm{GeV}$ cutoff for the matching corrections (about $40 \%$ relative to the matched result) has a relatively small impact in the $0 \mathrm{GeV}$ to $4 \mathrm{GeV}$ bin, is clearly visible in the first and last bins of $\phi^{*}$ and $\Delta \phi^{\gamma \gamma}$, respectively. We decided to keep these bins in our plot to demonstrate this effect which is unavoidable due to the large matching corrections, unless these can be calculated reliably also at small $q_{T}$.

Lastly, we show PDF uncertainties for the $q_{T}, \phi^{*}$ and $\Delta \phi$ distributions in figure 25 in the appendix A. Uncertainties are generally at the few percent level for each PDF set, but when taking into account the span of multiple PDF sets like CT18 and NNPDF3.1 they can reach up to $10 \%$. The ABMP16 set is undefined below scales of $4.47 \mathrm{GeV}$ and the LHAPDF 

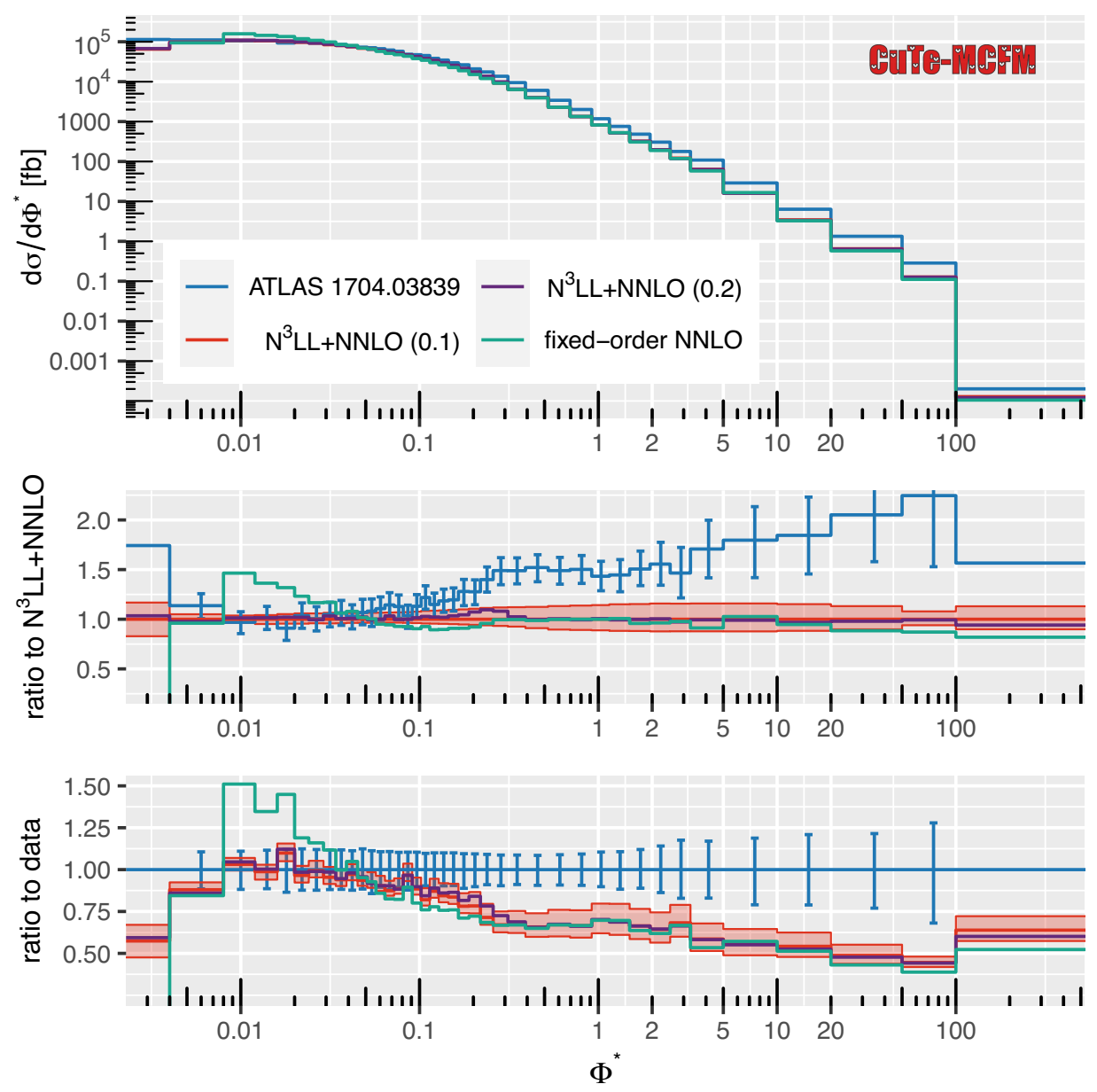

Figure 19. Comparison of the ATLAS measurement of the diphoton $\phi^{*}$ at $8 \mathrm{TeV}$ with predictions at $\mathrm{N}^{3} \mathrm{LL}+\mathrm{NNLO}$ including uncertainties associated with scale variation.

grid-based prediction therefore breaks down. In principle, as mentioned before, one could abandon the grid-based approach in this region and DGLAP evolve further downwards or just set $4.47 \mathrm{GeV}$ as a minimum scale value.

\subsection{Fiducial $Z \gamma$ production}

We now present results for fiducial $Z \gamma$ production in the decay channel $Z \rightarrow e^{+} e^{-}$in comparison with the $13 \mathrm{TeV}$ ATLAS measurement [133]. The fixed-order NNLO result in MCFM is based on ref. [21], but NNLO results have also been been computed in refs. [134, 135]. Results for $q_{T}$ resummation of $Z \gamma$ production at the same accuracy considered here have very recently been presented [59]. They are based on a different resummation framework [56] implemented in the RadiSH code.

We fully reproduce the NLO and NNLO fixed-order fiducial cross sections calculated in the ATLAS study [133] after applying the parton-to-particle factor $C_{\text {theory }}=0.934 \pm 0.005 . .^{5}$

\footnotetext{
${ }^{5}$ While ref. [133] gives a factor of 0.934 when electroweak corrections are included in the partonic prediction and 0.915 when they are not, we find that the former factor reproduces the fixed-order NLO and NNLO results in ref. [133] table 6. It seems possible that these factors have been mixed-up.
} 

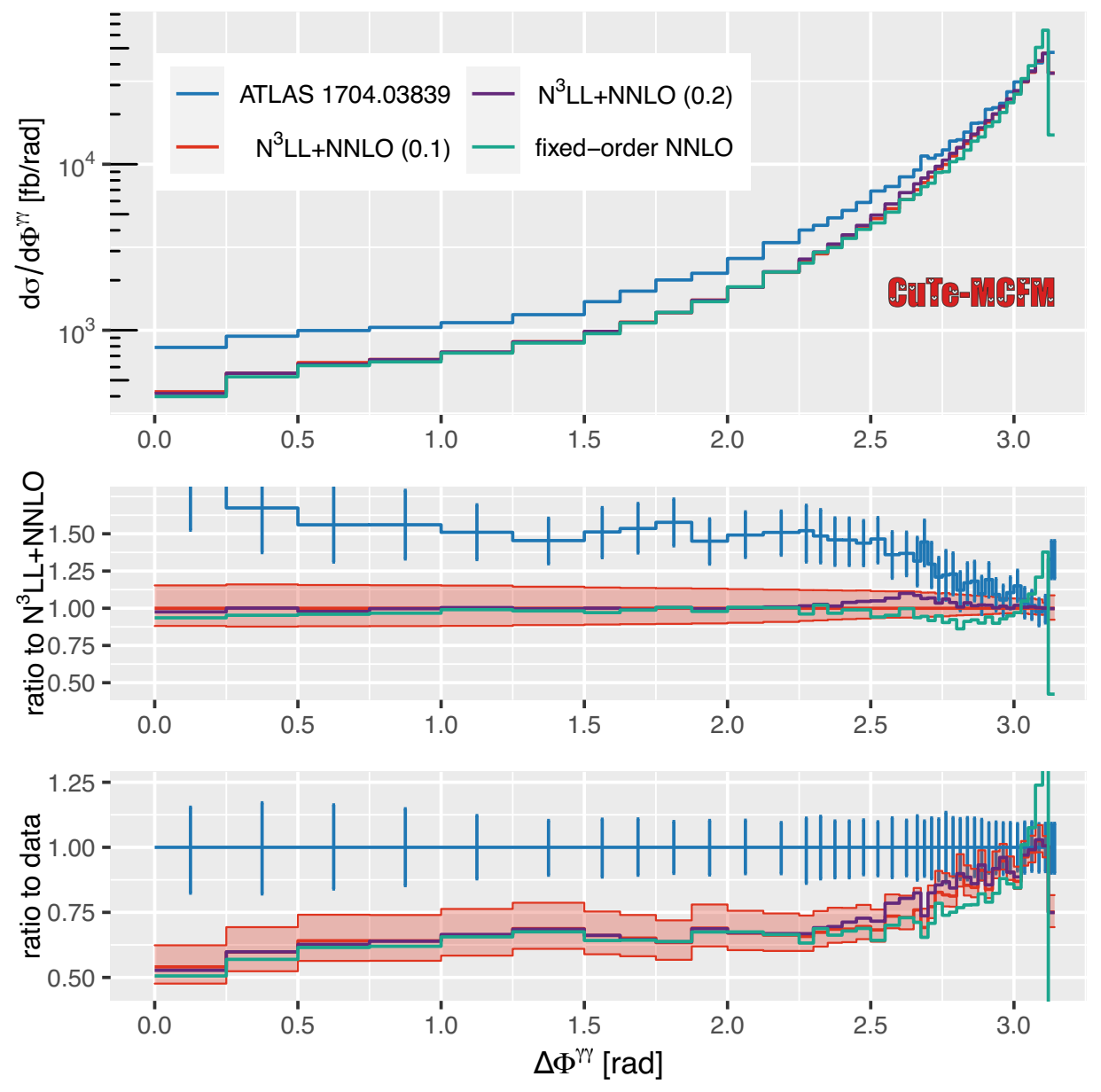

Figure 20. Comparison of the ATLAS measurement of the diphoton $\Delta \phi$ at $8 \mathrm{TeV}$ with predictions at $\mathrm{N}^{3} \mathrm{LL}+\mathrm{NNLO}$ including uncertainties associated with scale variation. The labels 0.1 and 0.2 in the plots refer to the value of $x^{\max }$.

We furthermore fully reproduce the presented fixed-order differential distributions at NNLO when including the differential parton-to-particle factors. Since we consider the use of parton-to-particle factors problematic, we do not directly include them in our results.

For $Z \gamma$ production two different contributions arise: an $s$-channel mode, where the photon is radiated from the charged leptons in the $Z$ decay, and a $t$-channel mode, where the photon is radiated from the initial state. The photon isolation enters differently in these channels. In the $s$-channel, the only isolation-cone power corrections are associated with gluon emission into the cone and suppressed by $R^{2}$, while the fragmentation part is absent. On the other hand, the $t$-channel has fragmentation contributions which are not suppressed by $R^{2}$. While the linear power corrections of the fragmentation contribution are asymptotically smaller than corrections from gluon emission (for isolation parameter $n>1$ ), they could still predominate for any reasonably small value of $q_{T} \rightarrow 0$ when $R \ll 1$, see discussion on page 11 and following. Since neither of these power corrections are included in our resummation, one expects larger matching corrections when the cuts allow for significant $t$-channel contributions. 

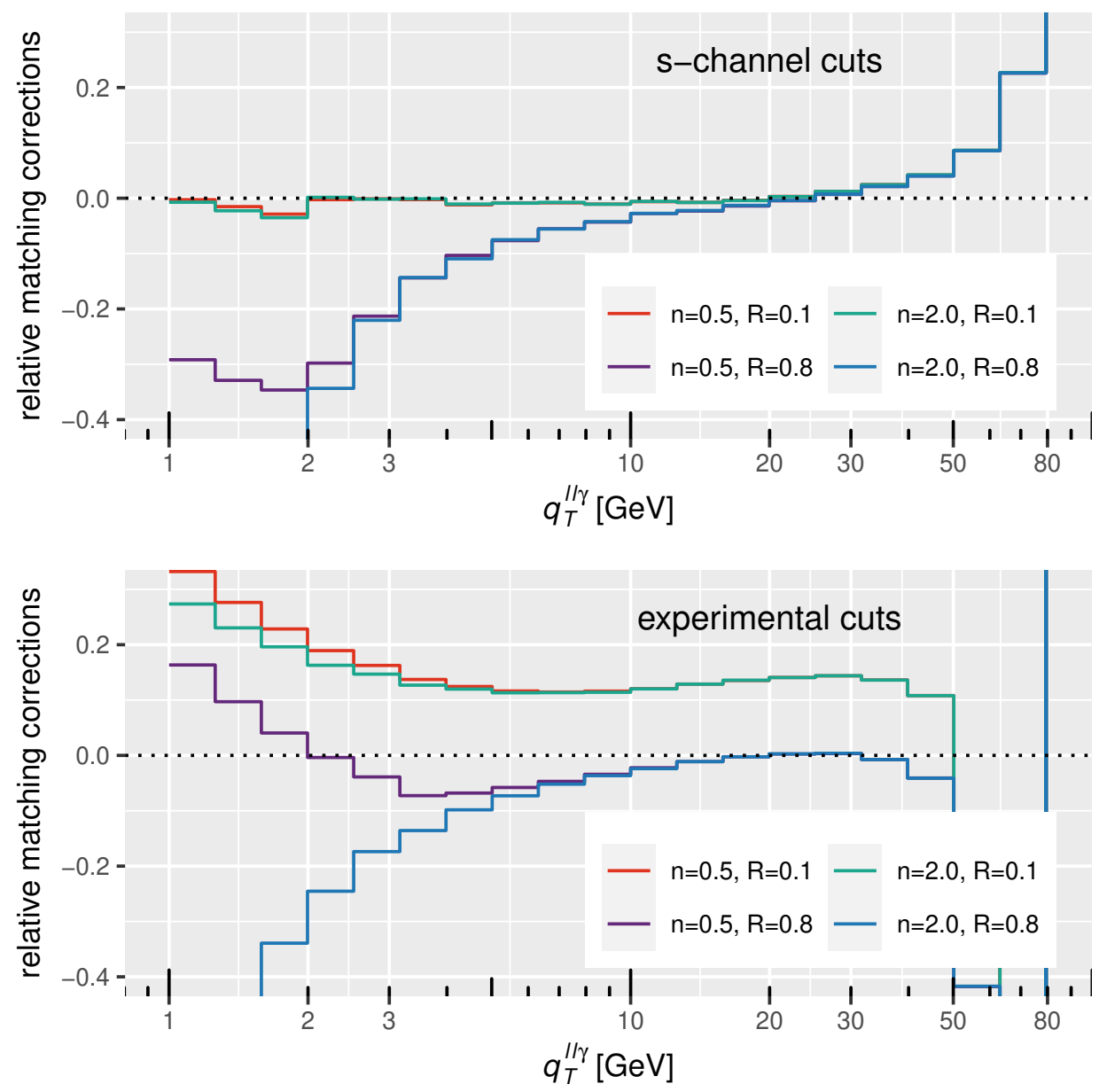

Figure 21. Matching corrections for $Z \gamma$ production at $\mathrm{N}^{2} \mathrm{LL}$, relative to the naively matched results for different choices of smooth-cone photon isolation parameters $n$ and $R_{0}$. The upper panel shows the results with $s$-channel benchmark cuts, see table 7 . The lower panel is relevant for the experimental cuts that enhance the $t$-channel contribution, see table 6 .

Impact of $s$-channel vs. $t$-channel contributions. The fiducial cuts chosen in the ATLAS study, see table 6 , almost entirely suppress the $s$-channel contribution to enhance the $Z$ peak in $m_{l^{+} l^{-}}$of the signal. This is primarily achieved by applying a selection cut $m_{l^{+} l^{-}}+m_{l^{+} l^{-} \gamma}>182 \mathrm{GeV}$, which can be nicely seen in figure 2 of ref. [133]. For those cuts, the matching corrections are large, as elaborated later on. To numerically test the impact of photon-isolation power corrections from $s$-channel and $t$-channel contributions we consider benchmark cuts as defined in table 7 . For these cuts we enhance the $s$-channel contribution by reversing the separation cut $m_{l^{+} l^{-}}+m_{l^{+} l^{-} \gamma}<182 \mathrm{GeV}$ and relaxing the photon and lepton $q_{T}$ cuts.

In figure 21 we consider the $\mathrm{N}^{2} \mathrm{LL}$ matching corrections relative to the naively matched result with $s$-channel benchmark cuts and experimental cuts for different choices of photon isolation parameters $R$ and $n$. For these benchmark cuts one observes exactly the behavior predicted for the gluonic corrections in eq. (2.16), namely negative effects scaling with 


\begin{tabular}{|l|c|}
\hline Leptons & $q_{T}^{l}>30 \mathrm{GeV}, 25 \mathrm{GeV},\left|\eta^{l}\right|<2.47$ \\
Photon & $q_{T}^{\gamma}>30 \mathrm{GeV}$ and $\left|\eta^{\gamma}\right|<2.37$ \\
Smooth-cone isolation & $\epsilon_{\gamma}=0.1, R=0.1, n=2$ \\
Separation & $m_{l^{+} l^{-}}>40 \mathrm{GeV}, m_{l^{+} l^{-}}+m_{l^{+} l^{-}}>182 \mathrm{GeV}, \Delta R(\gamma, l)>0.4$ \\
\hline
\end{tabular}

Table 6. Experimental cuts in $Z \gamma$ production with $Z \rightarrow e^{+} e^{-}$decay at a center of mass energy $\sqrt{s}=13 \mathrm{TeV}$.

\begin{tabular}{|l|c|}
\hline Leptons & $q_{T}^{l}>25 \mathrm{GeV}, 20 \mathrm{GeV},\left|\eta^{l}\right|<2.47$ \\
Photon & $q_{T}^{\gamma}>20 \mathrm{GeV}$ and $\left|\eta^{\gamma}\right|<2.37$ \\
Smooth-cone isolation & $\epsilon_{\gamma}=0.1, R$ and $n$ set individually \\
Separation & $m_{l^{+} l^{-}}>40 \mathrm{GeV}, m_{l^{+} l^{-}}+m_{l^{+} l^{-} \gamma}<182 \mathrm{GeV}, \Delta R(\gamma, l)>0.1$ \\
\hline
\end{tabular}

Table 7. Benchmark cuts enhancing the $s$-channel contribution in $Z \gamma$ production with $Z \rightarrow e^{+} e^{-}$ decay at a center of mass energy $\sqrt{s}=13 \mathrm{TeV}$.

$R^{2}$. This is different from diphoton production, where no $s$-channel mode exists and one cannot easily separate the power corrections associated with soft gluon emission from the fragmentation contribution.

The experimental cuts almost exclusively select the $t$-channel contribution. The nature of the power corrections changes and they become qualitatively similar to what we observed for diphoton production, except that they are smaller in size, because we only have a single photon in the final state. Even for $R=0.1$ the matching corrections are relatively large and positive around $10-20 \%$ and fully dominate over the Sudakov-suppressed resummed result towards $q_{T} \rightarrow 0$, since they scale linearly in $q_{T}$ and therefore approach a finite constant in the $q_{T}$ distribution. The matching corrections accidentally decrease for larger $R$ since the negative gluonic photon-isolation power corrections increase like $R^{2}$ and cancel against the fragmentation contributions. In all cases, the $n$-dependence only becomes relevant below at small $q_{T}<E_{T}^{\gamma, \max }$. For $\mathrm{N}^{3} \mathrm{LL}$ the same conclusions hold qualitatively and quantitatively.

\subsubsection{ATLAS measurements at $13 \mathrm{TeV}$}

Having demonstrated that matching corrections are at the percent to sub-percent level for the $s$-channel benchmark cuts and even moderate $q_{T} \lesssim 40 \mathrm{GeV}$, we now compare with the $13 \mathrm{TeV} \mathrm{Z} \gamma$ measurement by ATLAS [133] with fiducial cuts in table 6 . We use a central hard scale of $\mu_{h}=\sqrt{Q^{2}+q_{T}^{2}}$ and the CT14nnlo PDF set.

To see the effect of the experimental fiducial cuts at $\mathrm{N}^{3} \mathrm{LL}$ on the size of the matching corrections, we show the naively matched result and matching corrections in figure 22 . With a strong suppression of the $s$-channel contribution, the matching corrections at $\mathrm{N}^{3} \mathrm{LL}$ are at the order of $10-20 \%$. Fortunately the matching corrections are quite a bit smaller at $\mathrm{N}^{3} \mathrm{LL}$ than at $\mathrm{N}^{2} \mathrm{LL}$ for $q_{T}$ values in the few-GeV range.

To mitigate numerical issues due to a root in the matching corrections around $2 \mathrm{GeV}$ and required cancellations of more than 0.1 per-mille, we save computational resources 


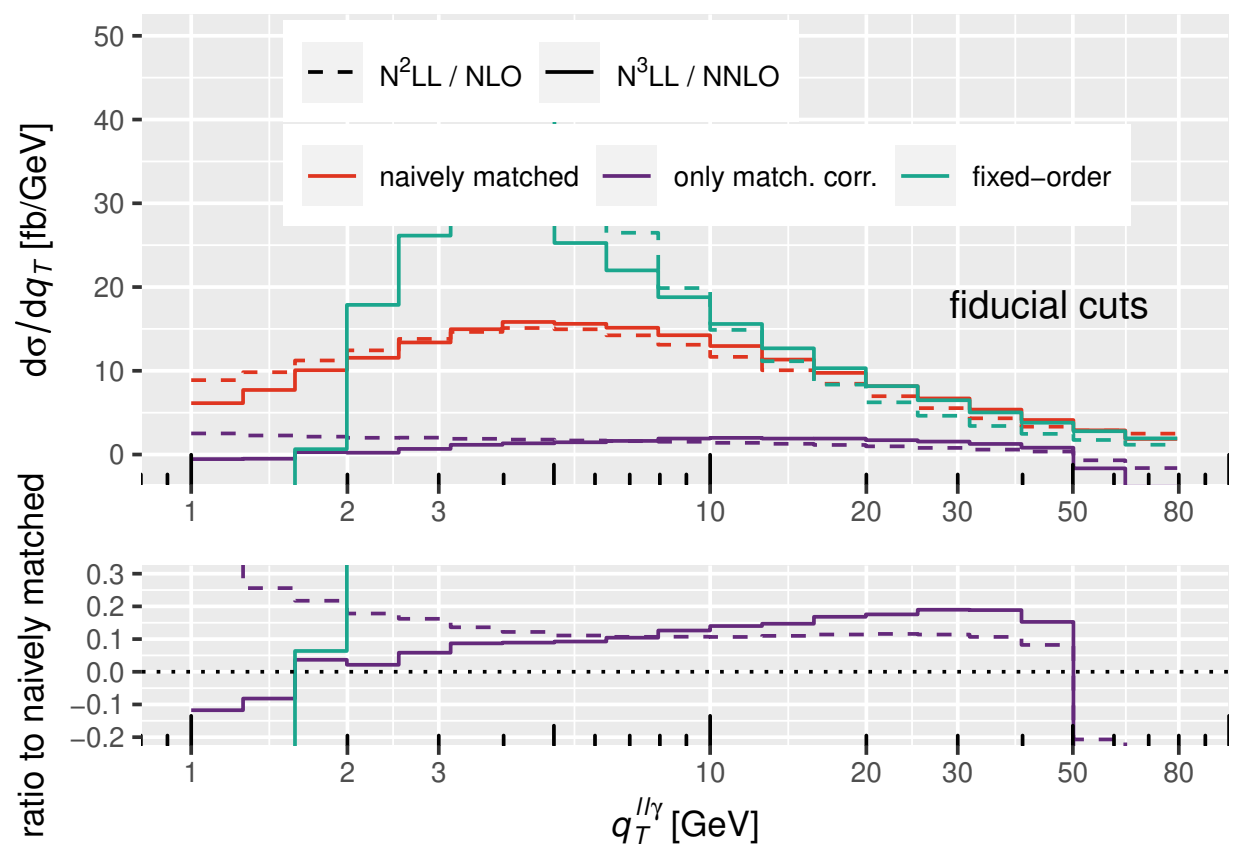

Figure 22. $Z\left(\rightarrow e^{+} e^{-}\right) \gamma$ transverse-momentum distribution with experimental fiducial cuts as in table 6 . Shown are results with naive matching, the matching corrections by themselves, and the fixed-order predictions, each at order $\alpha_{s}$ and $\alpha_{s}^{2}$, respectively. The bottom panel shows the ratio of the matching corrections relative to the naively matched result for each order in $\alpha_{s}$.

and cut off the matching corrections for the remaining $Z \gamma$ results below $2 \mathrm{GeV}$ and, as a consequence, neglect matching corrections of $\lesssim 5 \%$ below this value. The numerical issues can be seen in figure 22 for $q_{T}$ less than $2 \mathrm{GeV}$ as a discontinuity, or rather larger numerical noise.

To account for the safety cutoff on the matching corrections at $2 \mathrm{GeV}$, one should assign a larger uncertainty for the first few bins in the $q_{T}$ distribution. To estimate the effects for other variables, one can vary the cutoff value over the range of a few $\mathrm{GeV}$. We have done so for the $\Delta \phi$ distribution by increasing the cutoff from $2 \mathrm{GeV}$ to $5 \mathrm{GeV}$. The resulting changes are small compared to the other uncertainties which affect this distribution.

$\boldsymbol{q}_{\boldsymbol{T}}$ distribution. In figure 23 we show our predictions for the $q_{T}^{l l \gamma}$ distribution in comparison with the experimental data. The top panel shows the absolute distributions for data, resummed predictions matched to NNLO fixed-order results using transition function arguments $x^{\max }=0.01,0.1$ and $x^{\max }=0.2$, and the fixed-order NNLO prediction alone. The middle panel shows these distributions relative to the matched result with $x^{\max }=0.1$ to demonstrate matching effects to fixed order from the transition function. The bottom panel shows the results relative to the experimental data and includes uncertainties from scale-variation for the matched prediction $\left(x^{\max }=0.1\right)$ and experimental uncertainties for the experimental data.

The reason for choosing relatively small $x^{\max }$ for the transition function is that we want to minimize matching effects beyond $\sim 60 \mathrm{GeV}$, see figure 22 . The choice of $x^{\text {max }}=0.01$ 

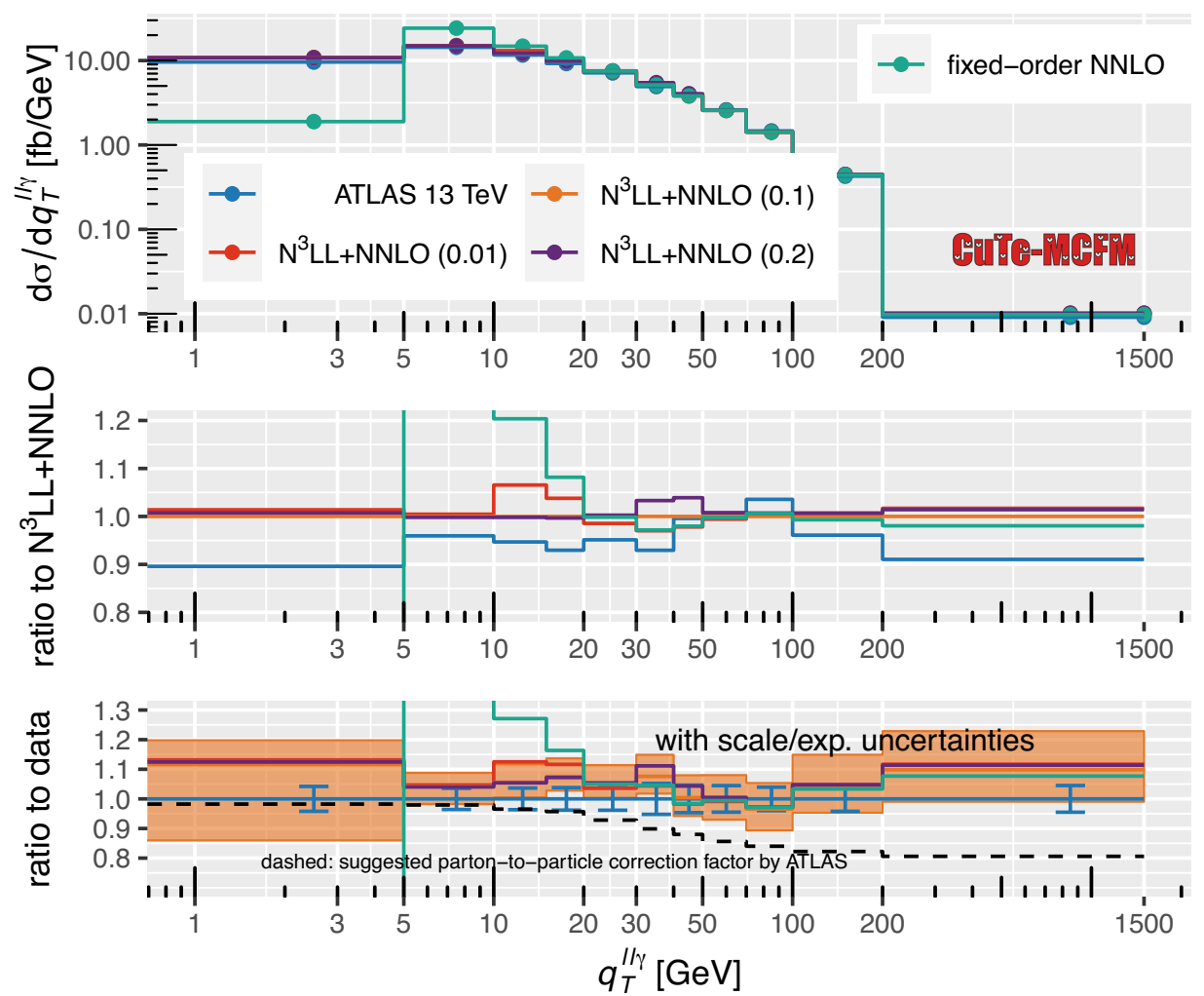

Figure 23. Comparison of $\mathrm{N}^{3} \mathrm{LL}+\mathrm{NNLO}$ predictions with the ATLAS measurement of $q_{T}^{l l \gamma}$ at $13 \mathrm{TeV}$. Note that this plot includes the first three bins individually that are only shown combined for the NNLO fixed-order comparison in ref. [133]. The labels $0.01,0.1$ and 0.2 in the plots refer to the value of $x^{\max }$.

performs the matching in the region of $\sim 10 \mathrm{GeV}$, where the validity of the fixed-order result is questionable and is entering the divergent regime. With $x^{\max }=0.1$ the transition region moves to between $30 \mathrm{GeV}$ and $50 \mathrm{GeV}$, while $x^{\max }=0.2$ stretches further into the region with large matching corrections. Taking $x^{\max }=0.1$ as a central choice, we estimate that the overall matching effects are about five percent around $30 \mathrm{GeV}$ to $50 \mathrm{GeV}$.

Despite the large matching corrections, the matched $\mathrm{N}^{3} \mathrm{LL}+\mathrm{NNLO}$ predictions show good agreement with data within scale uncertainties. The results using the resummation framework RadISH obtained in ref. [59] have similarly sized scale uncertainties and show a similar agreement with data. But unlike here, where we advocate to switch off the matching corrections with $x^{\max }=0.1$ in the region of $30 \mathrm{GeV}$ to $50 \mathrm{GeV}$, they state that resummation and matching are crucial also in the region $40 \mathrm{GeV} \lesssim q_{T}^{l l \gamma} \lesssim 200 \mathrm{GeV}$. While this choice could potentially have a positive effect on the agreement with data, the matching corrections and resummation spoil other kinematics, as we will see in the case of the $\Delta \phi$ observable. It is possible that the different matching procedure alleviates such issues, but the $\Delta \phi$ distribution has not been considered in ref. [59].

In the bottom panel we have additionally indicated the suggested parton-to-particle factor in the ATLAS study that, when applied, would decrease agreement. Note that 

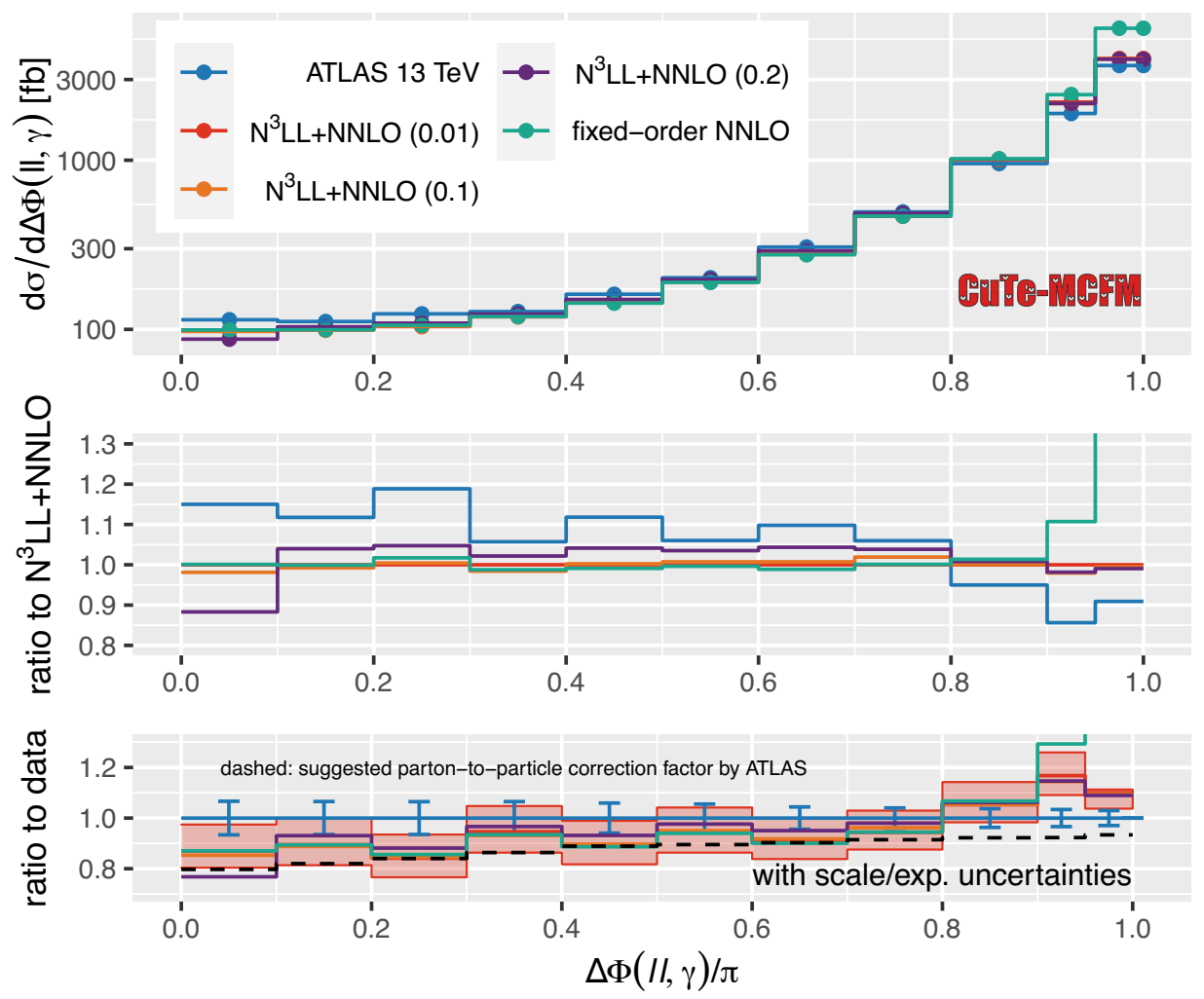

Figure 24. Comparison of $\mathrm{N}^{3} \mathrm{LL}+\mathrm{NNLO}$ predictions with ATLAS measurement of $\Delta \phi(l l, \gamma)$ at $13 \mathrm{TeV}$. Note that this plot includes the last two bins individually that are only shown combined for the NNLO fixed-order comparison in ref. [133]. The labels $0.01,0.1$ and 0.2 in the plots refer to the value of $x^{\max }$.

in addition to the large scale uncertainties ${ }^{6}$ of $10-20 \%$ also PDF uncertainties and unknown non-perturbative effects contribute, as well as a small effect from neglected matching corrections.

$\Delta \phi$ distribution. In figure 24 we compare our predictions with ATLAS data for the $\Delta \phi(l l, \gamma)$ observable. The first panel shows the absolute distributions, the middle panel the ratio to our matched prediction with $x^{\max }=0.01$, and the bottom panel the ratio to data with scale uncertainties for our predictions and experimental uncertainties for the measurement, respectively.

With this observable it becomes clear that with $x^{\max }=0.2$ we are tapping into a region where the resummation breaks down. This is most visible in the first bin, where the matched results should agree with the fixed-order prediction. Instead one sees a $10 \%$ difference. The first bin corresponds to azimuthally aligned $Z$ and $\gamma$, which due to the fiducial cuts have to recoil against at least $\sim 60 \mathrm{GeV}$. This is exactly the region where the resummation breaks down and which a transition function with $x^{\max }=0.2$ includes.

\footnotetext{
${ }^{6}$ The uncertainty is asymmetric in the first bin because we do not vary the resummation scale into the non-perturbative regime.
} 
While this is effect is most prominent for the first bin, also the larger azimuthal angles are affected at the percent-level. The effect worsens quickly with an even later transition.

The transitions with $x^{\max }=0.1$ and $x^{\max }=0.01$ ensure that the region where the resummation breaks down is excluded with negligible remaining effects. Therefore fixed-order and matched results agree up to $\Delta \phi=0.9 \cdot \pi$. The benefits of the $q_{T}$ resummation come into play in the last two bins, which are stabilized compared to the differentially diverging fixed-order prediction. Our prediction does not directly agree with the measurement at large $\Delta \phi$, but PDF uncertainties and non-perturbative effects are not yet included. In this region, the differential parton-to-particle correction used by ATLAS might be used as an estimate of non-perturbative effects. Including it would lead to better agreement with the data, at least in this region.

We present PDF uncertainties for the $q_{T}$ and $\Delta \phi$ distributions in figure 26 in the appendix A.

\section{Conclusions}

The transverse momentum of electroweak bosons is one of the cornerstone observables at the LHC and future colliders. It constitutes a precision probe of the Standard Model and is therefore one of the key observables to find or constrain new physics. The experimental precision reached today ranges from the per-mille level for $Z$ production, to the percent level for many diboson processes. While for Higgs production the uncertainties are currently still large, also these will diminish with more luminosity in the future. Such high-precision measurements are a huge challenge for theory that we help addressing with this study.

We presented a framework for $q_{T}$ resummation at $\mathrm{N}^{3} \mathrm{LL}+\mathrm{NNLO}$ for color-singlet processes based on a factorization theorem in SCET. Our implementation CuTe-MCFM provides precise predictions with uniform accuracy at small and large $q_{T}$ through a systematic power counting in $\alpha_{s}$ and large logarithms. Predictions can be calculated for the boson processes $W^{ \pm}, Z, H$, as well as for the diboson processes $\gamma \gamma, Z \gamma, Z H$ and $W^{ \pm} H$. These resummed and matched predictions are fully differential in the Born kinematics including decays and therefore also provide predictions for other observables benefiting from resummation at small $q_{T}$. Uncertainties from the perturbative QCD truncation, resummation, and PDFs can be evaluated efficiently using the possibility to pre-generate beam-function grids.

We first benchmarked our predictions for inclusive $Z$ and $H$ production with the code CuTe and then directly compared with fiducial experimental data for $Z, W, \gamma \gamma$ and $Z \gamma$ production. For $\gamma \gamma$ our results improve upon previous predictions at a lower logarithmic accuracy, and for $Z \gamma$ we presented novel results, previously only available at fixed-order accuracy. For $Z$ production, we observe excellent agreement at the few-percent level with the experimental measurements. The agreement is also quite good for $W$ and $Z \gamma$ production, while there are some tensions for diphoton production, which would likely ease after including $\alpha_{s}^{3}$ fixed-order corrections at large $q_{T}$. For Higgs production, where experimental uncertainties in the $q_{T}$ distribution are still large, we have presented results in the $H \rightarrow \gamma \gamma$ decay channel with realistic fiducial cuts as a first application. Furthermore, also the processes $W^{ \pm} H$ and $Z H$ can be calculated at $\mathrm{N}^{3} \mathrm{LL}+\mathrm{NNLO}$ with our code, which could 
become useful in the high-luminosity phase of the LHC. While observables related to $q_{T}$ were resummed at higher accuracy for these processes [136-138], transverse momentum resummation has to our knowledge only been carried out at NLL [139].

All of our results are shown with estimates of higher-order effects through scale variations of the hard scale, renormalization scale, factorization scale and resummation scale. We furthermore presented and discussed PDF uncertainties. We transition to fixed-order predictions at large $q_{T}$ through a simple sigmoid-type function which can easily be varied. Through this variation we estimated the uncertainty on the matching to fixed-order predictions.

We find that matching corrections are suppressed by $q_{T}^{2}$ for processes without photon isolation, if recoil effects are taken into account. For photon processes we showed that the necessary isolation requirements enhance the matching corrections and studied the form of the leading-order power corrections analytically and numerically. In the case of soft gluons radiated into the isolation cone, previous results are available that predict a power dependence on the smooth-cone isolation parameter $n$, which we confirm. For the power corrections associated with quark fragmentation, we find that they are always first order in $q_{T}$ and are not suppressed by the size of the isolation cone. Therefore, the resulting presence of large power corrections can make it difficult to find a window in which the fixed-order and resummed predictions are both valid and can be matched reliably.

While our implementation is part of MCFM, the resummation code is highly modular and could easily be decoupled and interfaced to other codes supplying the fixed-order process-dependent pieces, i.e. the hard function for the resummation itself, and the process with additional radiation recoiling at large $q_{T}$. Our code CuTe-MCFM will be made publicly available shortly.

Using our existing framework one could, with limited effort, match with $\alpha_{s}^{3}$ predictions at large $q_{T}[98,120,140-142]$ to provide predictions at $\mathrm{N}^{3} \mathrm{LL}^{\prime}+\mathrm{N}^{3} \mathrm{LO}$. To do so, one will need to implement the recently computed three-loop beam functions [143, 144]. However, apart from the case of Higgs and Drell-Yan production this would mean neglecting the $\alpha_{s}^{3}$ hard function. We could furthermore easily include non-perturbative effects either through a form-factor modification in the resummation or through swapping out the beam functions for transverse-momentum dependent PDFs. With this, even fits for these generalized PDFs can be envisioned as long as precise control over matching corrections is maintained when they are sizeable at small $q_{T}$. The inclusion of electroweak effects in the resummation and in fixed-order results is another issue that should be tackled together with other higherorder effects. For Higgs production the inclusion of heavy-quark mass effects is another interesting avenue to pursue.

\section{Acknowledgments}

We would like to thank John Campbell for useful suggestions (electroweak corrections), Markus Ebert for providing additional details on the results in ref. [86] and Dingyu Shao for discussions on the resummation of $\Delta \Phi$. The research of T.B. is supported by the Swiss National Science Foundation (SNF) under grant 200020_182038. This work was supported 
by the U.S. Department of Energy under award No. DE-SC0008347. This document was prepared using the resources of the Fermi National Accelerator Laboratory (Fermilab), a U.S. Department of Energy, Office of Science, HEP User Facility. Fermilab is managed by Fermi Research Alliance, LLC (FRA), acting under Contract No. DE-AC02-07CH11359.

\section{A PDF uncertainties}

In this appendix we present plots with PDF uncertainties for diphoton and for $Z \gamma$ production. We evaluate these for the following NNLO PDF sets with fixed value of $\alpha_{s}\left(m_{Z}\right)=0.118:$ ABMP16 [102], CT14 [103], CT18 [104], MMHT2014 [105], NNPDF30 [107] and NNPDF31 [108] interfaced to LHAPDF [96]. Overall the PDF uncertainties are broadly at the few percent level, but can become larger when taking into account multiple sets. The individual central values are mostly compatible within mutual uncertainties. The ABMP16 set is not defined below scales of $\mu=4.47 \mathrm{GeV}$ and breaks down with the default grid-based interpolation in LHAPDF. In principle one could switch to DGLAP evolution to circumvent this or use a larger minimum scale of $4.47 \mathrm{GeV}$ in CuTe-MCFM. 

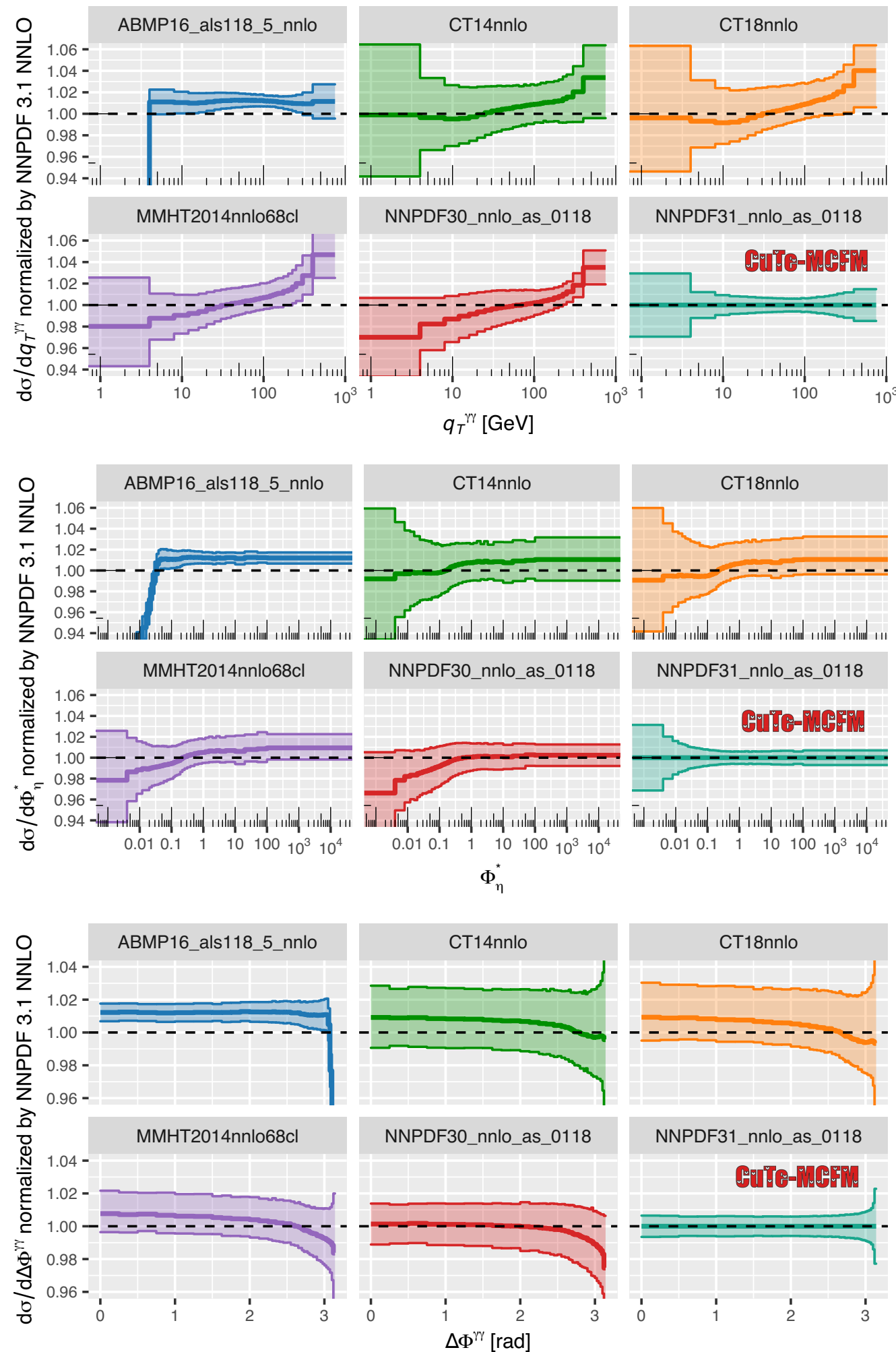

Figure 25. PDF uncertainties for the $q_{T}, \phi^{*}$ and $\Delta \phi$ distributions in figures 18,19 and 20. The ABMP16 set is not defined below $\mu=4.47 \mathrm{GeV}$, which can be seen as breakdown in the prediction. 


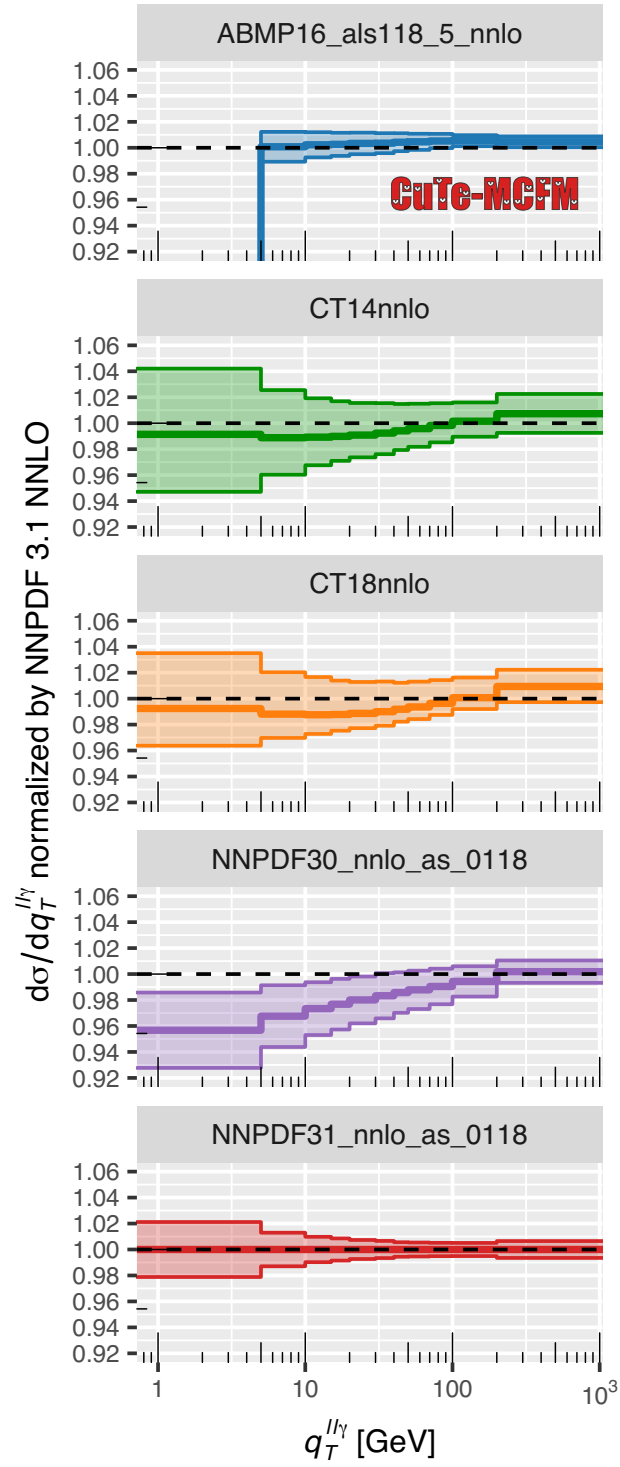

(a) For $q_{T}$ distribution

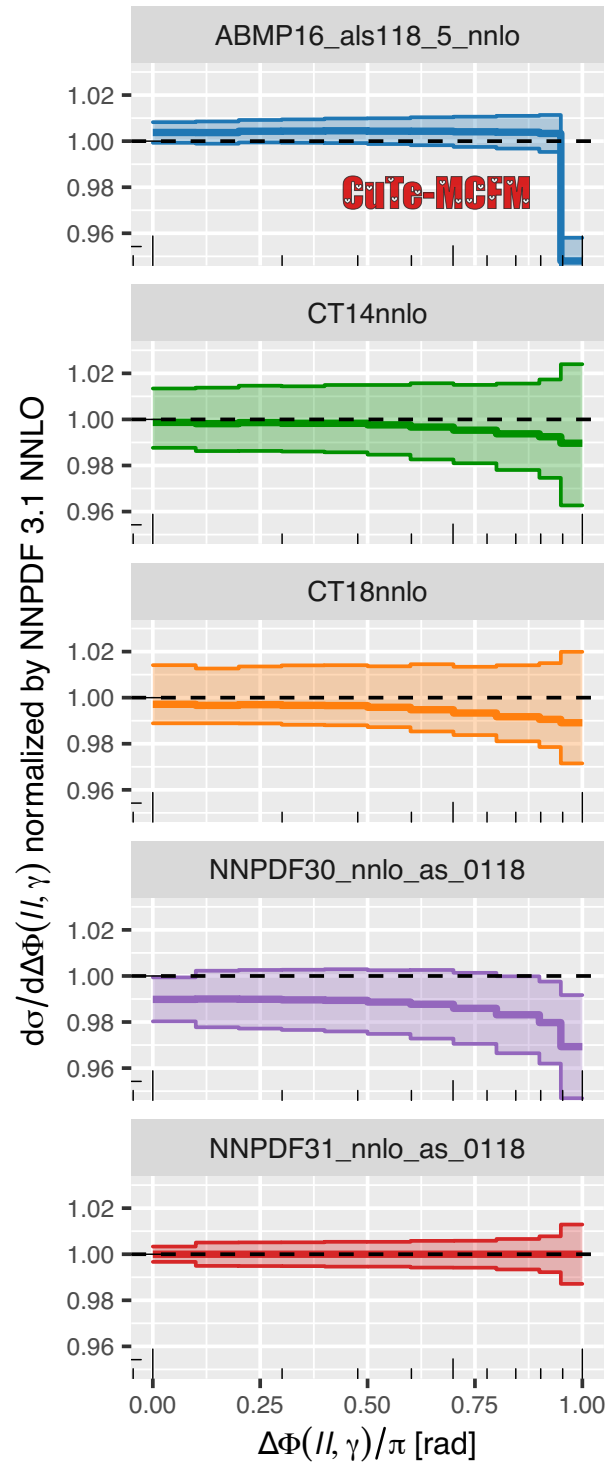

(b) For $\Delta \phi$ distribution

Figure 26. PDF uncertainties for the $q_{T}$ (a) and $\Delta \phi$ (b) distributions in $Z \gamma$ production corresponding to the distributions with experimental selection cuts in figure 23 and figure 24 , respectively. The ABMP16 grid is not defined below $\mu=4.47 \mathrm{GeV}$, which can be seen as breakdown in the prediction. 
Open Access. This article is distributed under the terms of the Creative Commons Attribution License (CC-BY 4.0), which permits any use, distribution and reproduction in any medium, provided the original author(s) and source are credited.

\section{References}

[1] ATLAS collaboration, Measurement of the $W$-boson mass in pp collisions at $\sqrt{s}=7 \mathrm{TeV}$ with the ATLAS detector, Eur. Phys. J. C 78 (2018) 110 [Erratum ibid. 78 (2018) 898] [arXiv: 1701.07240] [INSPIRE].

[2] A. Banfi, S. Redford, M. Vesterinen, P. Waller and T.R. Wyatt, Optimisation of variables for studying dilepton transverse momentum distributions at hadron colliders, Eur. Phys. J. C 71 (2011) 1600 [arXiv: 1009.1580] [InSPIRE].

[3] R. Boughezal, A. Guffanti, F. Petriello and M. Ubiali, The impact of the LHC Z-boson transverse momentum data on PDF determinations, JHEP 07 (2017) 130 [arXiv: 1705.00343] [INSPIRE].

[4] CMS collaboration, Measurement of the $W^{+} W^{-}$cross section in pp collisions at $\sqrt{s}=8$ $\mathrm{TeV}$ and limits on anomalous gauge couplings, Eur. Phys. J. C 76 (2016) 401 [arXiv: 1507. 03268] [INSPIRE].

[5] ATLAS collaboration, Measurements of $W^{ \pm} Z$ production cross sections in pp collisions at $\sqrt{s}=8 \mathrm{TeV}$ with the ATLAS detector and limits on anomalous gauge boson self-couplings, Phys. Rev. D 93 (2016) 092004 [arXiv: 1603.02151] [INSPIRE].

[6] ATLAS collaboration, Measurement of inclusive and differential cross sections in the $H \rightarrow Z Z^{*} \rightarrow 4 \ell$ decay channel in pp collisions at $\sqrt{s}=13 \mathrm{TeV}$ with the ATLAS detector, JHEP 10 (2017) 132 [arXiv:1708.02810] [INSPIRE].

[7] ATLAS collaboration, $Z Z \rightarrow \ell^{+} \ell^{-} \ell^{\prime+} \ell^{\prime-}$ cross-section measurements and search for anomalous triple gauge couplings in $13 \mathrm{TeV}$ pp collisions with the ATLAS detector, Phys. Rev. D 97 (2018) 032005 [arXiv: 1709.07703] [INSPIRE].

[8] CMS collaboration, Measurements of the $p p \rightarrow Z Z$ production cross section and the $Z \rightarrow 4 \ell$ branching fraction, and constraints on anomalous triple gauge couplings at $\sqrt{s}=13$ TeV, Eur. Phys. J. C 78 (2018) 165 [Erratum ibid. 78 (2018) 515] [arXiv: 1709.08601] [INSPIRE].

[9] CMS collaboration, Measurements of the $p p \rightarrow W Z$ inclusive and differential production cross section and constraints on charged anomalous triple gauge couplings at $\sqrt{s}=13 \mathrm{TeV}$, JHEP 04 (2019) 122 [arXiv:1901.03428] [INSPIRE].

[10] ATLAS collaboration, Measurement of the four-lepton invariant mass spectrum in $13 \mathrm{TeV}$ proton-proton collisions with the ATLAS detector, JHEP 04 (2019) 048 [arXiv: 1902.05892] [INSPIRE].

[11] CMS collaboration, Search for anomalous triple gauge couplings in $W W$ and $W Z$ production in lepton + jet events in proton-proton collisions at $\sqrt{s}=13 \mathrm{TeV}, J H E P 12$ (2019) 062 [arXiv: 1907.08354] [inSPIRE].

[12] ATLAS collaboration, Measurement of $W^{ \pm} Z$ production cross sections and gauge boson polarisation in pp collisions at $\sqrt{s}=13 \mathrm{TeV}$ with the ATLAS detector, Eur. Phys. J. C 79 (2019) 535 [arXiv: 1902.05759] [INSPIRE].

[13] K. Hagiwara, R.D. Peccei, D. Zeppenfeld and K. Hikasa, Probing the Weak Boson Sector in $e^{+} e^{-} \rightarrow W^{+} W^{-}$, Nucl. Phys. B 282 (1987) 253 [INSPIRE]. 
[14] K. Hagiwara, J. Woodside and D. Zeppenfeld, Measuring the $W W Z$ coupling at the Tevatron, Phys. Rev. D 41 (1990) 2113 [inSPIRE].

[15] C. Frye, M. Freytsis, J. Scholtz and M.J. Strassler, Precision diboson observables for the LHC, JHEP 03 (2016) 171 [arXiv:1510.08451] [InSPIRE].

[16] R. Franceschini, G. Panico, A. Pomarol, F. Riva and A. Wulzer, Electroweak precision tests in high-energy diboson processes, JHEP 02 (2018) 111 [arXiv:1712.01310] [INSPIRE].

[17] C. Grojean, M. Montull and M. Riembau, Diboson at the LHC vs. LEP, JHEP 03 (2019) 020 [arXiv: 1810.05149 ] [INSPIRE].

[18] J. Baglio, S. Dawson, S. Homiller, S.D. Lane and I.M. Lewis, Validity of standard model EFT studies of VH and VV production at NLO, Phys. Rev. D 101 (2020) 115004 [arXiv: 2003. 07862] [INSPIRE].

[19] R. Boughezal et al., Color singlet production at NNLO in MCFM, Eur. Phys. J. C 77 (2017) 7 [arXiv: 1605. 08011] [inSPIRE].

[20] J.M. Campbell, R.K. Ellis and C. Williams, Associated production of a Higgs boson at NNLO, JHEP 06 (2016) 179 [arXiv:1601.00658] [INSPIRE].

[21] J.M. Campbell, T. Neumann and C. Williams, $Z \gamma$ production at NNLO including anomalous couplings, JHEP 11 (2017) 150 [arXiv:1708.02925] [INSPIRE].

[22] J. Campbell and T. Neumann, Precision phenomenology with MCFM, JHEP 12 (2019) 034 [arXiv: 1909.09117] [INSPIRE].

[23] T. Becher and M. Neubert, Drell-Yan production at small $q_{T}$, transverse parton distributions and the collinear anomaly, Eur. Phys. J. C 71 (2011) 1665 [arXiv: 1007.4005] [INSPIRE].

[24] T. Becher, M. Neubert and D. Wilhelm, Electroweak gauge-boson production at small $q_{T}$ : infrared safety from the collinear anomaly, JHEP 02 (2012) 124 [arXiv:1109.6027] [INSPIRE].

[25] T. Becher, M. Neubert and D. Wilhelm, Higgs-boson production at small transverse momentum, JHEP 05 (2013) 110 [arXiv:1212.2621] [INSPIRE].

[26] T. Becher and M. Hager, Event-based transverse momentum resummation, Eur. Phys. J. C 79 (2019) 665 [arXiv: 1904.08325] [INSPIRE].

[27] J.C. Collins, D.E. Soper and G.F. Sterman, Transverse momentum distribution in Drell-Yan pair and $W$ and $Z$ boson production, Nucl. Phys. B 250 (1985) 199 [INSPIRE].

[28] C.W. Bauer, S. Fleming, D. Pirjol and I.W. Stewart, An Effective field theory for collinear and soft gluons: Heavy to light decays, Phys. Rev. D 63 (2001) 114020 [hep-ph/0011336] [inSPIRE].

[29] C.W. Bauer, D. Pirjol and I.W. Stewart, Soft collinear factorization in effective field theory, Phys. Rev. D 65 (2002) 054022 [hep-ph/0109045] [INSPIRE].

[30] M. Beneke, A.P. Chapovsky, M. Diehl and T. Feldmann, Soft collinear effective theory and heavy to light currents beyond leading power, Nucl. Phys. B 643 (2002) 431 [hep-ph/0206152] [INSPIRE].

[31] T. Becher, A. Broggio and A. Ferroglia, Introduction to soft-collinear effective theory, Lecture Notes in Physics volume 896, Springer, Germany (2015) [arXiv:1410.1892] [INSPIRE]. 
[32] T. Becher, Soft-collinear effective theory, Les Houches Lect. Notes 108 (2020) [arXiv: 1803.04310] [INSPIRE].

[33] T. Cohen, As scales become separated: lectures on effective field theory, PoS (TASI2018)011 [arXiv:1903.03622] [INSPIRE].

[34] Y. Gao, C.S. Li and J.J. Liu, Transverse momentum resummation for Higgs production in soft-collinear effective theory, Phys. Rev. D 72 (2005) 114020 [hep-ph/0501229] [INSPIRE].

[35] A. Idilbi, X.-d. Ji and F. Yuan, Transverse momentum distribution through soft-gluon resummation in effective field theory, Phys. Lett. B 625 (2005) 253 [hep-ph/0507196] [INSPIRE].

[36] S. Mantry and F. Petriello, Factorization and resummation of Higgs boson differential distributions in soft-collinear effective Theory, Phys. Rev. D 81 (2010) 093007 [arXiv:0911.4135] [INSPIRE].

[37] J.-Y. Chiu, A. Jain, D. Neill and I.Z. Rothstein, A formalism for the systematic treatment of rapidity logarithms in quantum field theory, JHEP 05 (2012) 084 [arXiv:1202.0814] [INSPIRE].

[38] J.-y. Chiu, A. Jain, D. Neill and I.Z. Rothstein, The rapidity renormalization group, Phys. Rev. Lett. 108 (2012) 151601 [arXiv:1104.0881] [INSPIRE].

[39] G. Bozzi, S. Catani, G. Ferrera, D. de Florian and M. Grazzini, Production of Drell-Yan lepton pairs in hadron collisions: Transverse-momentum resummation at next-to-next-to-leading logarithmic accuracy, Phys. Lett. B 696 (2011) 207 [arXiv: 1007.2351] [INSPIRE].

[40] S. Catani, D. de Florian, G. Ferrera and M. Grazzini, Vector boson production at hadron colliders: transverse-momentum resummation and leptonic decay, JHEP 12 (2015) 047 [arXiv: 1507.06937] [INSPIRE].

[41] S. Camarda et al., DYTurbo: fast predictions for Drell-Yan processes, Eur. Phys. J. C 80 (2020) 251 [Erratum ibid. 80 (2020) 440] [arXiv: 1910.07049] [INSPIRE].

[42] F. Coradeschi and T. Cridge, reSolve - A transverse momentum resummation tool, Comput. Phys. Commun. 238 (2019) 262 [arXiv:1711.02083] [inSPIRE].

[43] V. Bertone, I. Scimemi and A. Vladimirov, Extraction of unpolarized quark transverse momentum dependent parton distributions from Drell-Yan/Z-boson production, JHEP 06 (2019) 028 [arXiv: 1902.08474] [INSPIRE].

[44] A. Bacchetta et al., Transverse-momentum-dependent parton distributions up to $N^{3} L L$ from Drell-Yan data, JHEP 07 (2020) 117 [arXiv:1912.07550] [INSPIRE].

[45] M.A. Ebert, J.K.L. Michel, I.W. Stewart and F.J. Tackmann, Drell-Yan $q_{T}$ Resummation of Fiducial Power Corrections at $N^{3} L L$, arXiv:2006.11382 [INSPIRE].

[46] D. de Florian, G. Ferrera, M. Grazzini and D. Tommasini, Higgs boson production at the LHC: transverse momentum resummation effects in the $H \rightarrow \gamma \gamma, H \rightarrow W W \rightarrow l \nu l \nu$ and $H \rightarrow Z Z \rightarrow 4 l$ decay modes, JHEP 06 (2012) 132 [arXiv:1203.6321] [INSPIRE].

[47] M. Grazzini and H. Sargsyan, Heavy-quark mass effects in Higgs boson production at the LHC, JHEP 09 (2013) 129 [arXiv:1306.4581] [INSPIRE].

[48] J. Wang, C.S. Li, Z. Li, C.P. Yuan and H.T. Li, Improved resummation prediction on Higgs production at hadron colliders, Phys. Rev. D 86 (2012) 094026 [arXiv:1205.4311] [INSPIRE]. 
[49] C. Balázs and C.P. Yuan, Soft gluon effects on lepton pairs at hadron colliders, Phys. Rev. D 56 (1997) 5558 [hep-ph/9704258] [INSPIRE].

[50] G.A. Ladinsky and C.P. Yuan, The nonperturbative regime in QCD resummation for gauge boson production at hadron colliders, Phys. Rev. D 50 (1994) 4239 [hep-ph/9311341] [INSPIRE].

[51] M. Grazzini, S. Kallweit, D. Rathlev and M. Wiesemann, Transverse-momentum resummation for vector-boson pair production at NNLL+NNLO, JHEP 08 (2015) 154 [arXiv: 1507.02565] [INSPIRE].

[52] A. Banfi, G.P. Salam and G. Zanderighi, Semi-numerical resummation of event shapes, JHEP 01 (2002) 018 [hep-ph/0112156] [INSPIRE].

[53] A. Banfi, G.P. Salam and G. Zanderighi, Principles of general final-state resummation and automated implementation, JHEP 03 (2005) 073 [hep-ph/0407286] [INSPIRE].

[54] A. Banfi, H. McAslan, P.F. Monni and G. Zanderighi, A general method for the resummation of event-shape distributions in $e^{+} e^{-}$annihilation, JHEP 05 (2015) 102 [arXiv: 1412.2126] [INSPIRE].

[55] P.F. Monni, E. Re and P. Torrielli, Higgs transverse-momentum resummation in direct space, Phys. Rev. Lett. 116 (2016) 242001 [arXiv:1604.02191] [INSPIRE].

[56] W. Bizon, P.F. Monni, E. Re, L. Rottoli and P. Torrielli, Momentum-space resummation for transverse observables and the Higgs $p_{\perp}$ at $N^{3} L L+N N L O$, JHEP 02 (2018) 108 [arXiv: 1705. 09127] [INSPIRE].

[57] M. Grazzini, S. Kallweit and M. Wiesemann, Fully differential NNLO computations with MATRIX, Eur. Phys. J. C 78 (2018) 537 [arXiv:1711.06631] [InSPIRE].

[58] S. Kallweit, E. Re, L. Rottoli and M. Wiesemann, Accurate single- and double-differential resummation of colour-singlet processes with MATRIX + RADISH: $W^{+} W^{-}$production at the LHC, JHEP 12 (2020) 147 [arXiv:2004.07720] [INSPIRE].

[59] M. Wiesemann, L. Rottoli and P. Torrielli, The $Z \gamma$ transverse-momentum spectrum at NNLO+N $N^{3} L L$, Phys. Lett. B 809 (2020) 135718 [arXiv: 2006.09338] [INSPIRE].

[60] X. Chen et al., Precise QCD description of the Higgs boson transverse momentum spectrum, Phys. Lett. B 788 (2019) 425 [arXiv: 1805.00736] [INSPIRE].

[61] W. Bizoń et al., Fiducial distributions in Higgs and Drell-Yan production at $N^{3} L L+N N L O$, JHEP 12 (2018) 132 [arXiv: 1805.05916] [INSPIRE].

[62] W. Bizon et al., The transverse momentum spectrum of weak gauge bosons at $N^{3} L L+$ NNLO, Eur. Phys. J. C 79 (2019) 868 [arXiv:1905.05171] [InSPIRE].

[63] A. Buckley et al., General-purpose event generators for LHC physics, Phys. Rept. 504 (2011) 145 [arXiv: 1101.2599] [INSPIRE].

[64] S. Alioli et al., Combining Higher-Order Resummation with Multiple NLO Calculations and Parton Showers in GENEVA, JHEP 09 (2013) 120 [arXiv:1211.7049] [INSPIRE].

[65] M.A. Ebert and F.J. Tackmann, Resummation of transverse momentum distributions in distribution space, JHEP 02 (2017) 110 [arXiv:1611.08610] [INSPIRE].

[66] S. Catani, L. Trentadue, G. Turnock and B.R. Webber, Resummation of large logarithms in $e^{+} e^{-}$event shape distributions, Nucl. Phys. B 407 (1993) 3 [INSPIRE].

[67] S. Catani and M. Grazzini, QCD transverse-momentum resummation in gluon fusion processes, Nucl. Phys. B 845 (2011) 297 [arXiv:1011.3918] [InSPIRE]. 
[68] I.W. Stewart, F.J. Tackmann and W.J. Waalewijn, $N$-jettiness: an inclusive event shape to veto jets, Phys. Rev. Lett. 105 (2010) 092002 [arXiv: 1004.2489] [INSPIRE].

[69] T. Becher and M. Neubert, On the structure of infrared singularities of gauge-theory amplitudes, JHEP 06 (2009) 081 [Erratum ibid. 11 (2013) 024] [arXiv:0903.1126] [INSPIRE].

[70] S. Moch, B. Ruijl, T. Ueda, J.A.M. Vermaseren and A. Vogt, On quartic colour factors in splitting functions and the gluon cusp anomalous dimension, Phys. Lett. B 782 (2018) 627 [arXiv: 1805. 09638] [INSPIRE].

[71] J.M. Henn, G.P. Korchemsky and B. Mistlberger, The full four-loop cusp anomalous dimension in $\mathcal{N}=4$ super Yang-Mills and QCD, JHEP 04 (2020) 018 [arXiv:1911.10174] [INSPIRE].

[72] A. von Manteuffel, E. Panzer and R.M. Schabinger, Cusp and collinear anomalous dimensions in four-loop QCD from form factors, Phys. Rev. Lett. 124 (2020) 162001 [arXiv: 2002.04617] [INSPIRE].

[73] Y. Li and H.X. Zhu, Bootstrapping rapidity anomalous dimensions for transverse-momentum resummation, Phys. Rev. Lett. 118 (2017) 022004 [arXiv: 1604.01404] [INSPIRE].

[74] A.A. Vladimirov, Correspondence between soft and rapidity anomalous dimensions, Phys. Rev. Lett. 118 (2017) 062001 [arXiv:1610.05791] [INSPIRE].

[75] G. Parisi and R. Petronzio, Small transverse momentum distributions in hard processes, Nucl. Phys. B 154 (1979) 427 [InSPIRE].

[76] T. Becher and M. Neubert, Infrared singularities of scattering amplitudes and $N^{3} L L$ resummation for $n$-jet processes, JHEP 01 (2020) 025 [arXiv: 1908.11379] [INSPIRE].

[77] T. Gehrmann, T. Lubbert and L.L. Yang, Transverse parton distribution functions at next-to-next-to-leading order: the quark-to-quark case, Phys. Rev. Lett. 109 (2012) 242003 [arXiv: 1209.0682] [INSPIRE].

[78] T. Gehrmann, T. Luebbert and L.L. Yang, Calculation of the transverse parton distribution functions at next-to-next-to-leading order, JHEP 06 (2014) 155 [arXiv:1403.6451] [INSPIRE].

[79] D. Bertolini, M.P. Solon and J.R. Walsh, Integrated and differential accuracy in resummed cross sections, Phys. Rev. D 95 (2017) 054024 [arXiv:1701.07919] [InSPIRE].

[80] A.A. Penin, High-energy limit of quantum electrodynamics beyond Sudakov approximation, Phys. Lett. B $\mathbf{7 4 5}$ (2015) 69 [Erratum ibid. 751 (2015) 596] [Erratum ibid. 771 (2017) 633] [arXiv: 1412.0671] [INSPIRE].

[81] I. Moult, I.W. Stewart, G. Vita and H.X. Zhu, First Subleading Power Resummation for Event Shapes, JHEP 08 (2018) 013 [arXiv:1804.04665] [INSPIRE].

[82] M. Beneke, M. Garny, S. Jaskiewicz, R. Szafron, L. Vernazza and J. Wang, Leading-logarithmic threshold resummation of Higgs production in gluon fusion at next-to-leading power, JHEP 01 (2020) 094 [arXiv: 1910.12685] [INSPIRE].

[83] N. Bahjat-Abbas et al., Diagrammatic resummation of leading-logarithmic threshold effects at next-to-leading power, JHEP 11 (2019) 002 [arXiv: 1905.13710] [INSPIRE].

[84] Z.L. Liu, B. Mecaj, M. Neubert and X. Wang, Factorization at subleading power and endpoint divergences in soft-collinear effective theory, arXiv:2009.04456 [INSPIRE]. 
[85] Z.L. Liu, B. Mecaj, M. Neubert and X. Wang, Factorization at subleading power and endpoint divergences in $h \rightarrow \gamma \gamma$ decay. Part II. Renormalization and scale evolution, JHEP 01 (2021) 077 [arXiv: 2009.06779] [inSPIRE].

[86] M.A. Ebert and F.J. Tackmann, Impact of isolation and fiducial cuts on $q_{T}$ and $N$-jettiness subtractions, JHEP 03 (2020) 158 [arXiv:1911.08486] [INSPIRE].

[87] A. Banfi, M. Dasgupta and Y. Delenda, Azimuthal decorrelations between QCD jets at all orders, Phys. Lett. B 665 (2008) 86 [arXiv:0804.3786] [INSPIRE].

[88] A. Banfi, M. Dasgupta and S. Marzani, QCD predictions for new variables to study dilepton transverse momenta at hadron colliders, Phys. Lett. B 701 (2011) 75 [arXiv:1102.3594] [INSPIRE].

[89] A. Banfi, M. Dasgupta, S. Marzani and L. Tomlinson, Predictions for Drell-Yan $\phi^{*}$ and $Q_{T}$ observables at the LHC, Phys. Lett. B 715 (2012) 152 [arXiv:1205.4760] [INSPIRE].

[90] S. Frixione, Isolated photons in perturbative QCD, Phys. Lett. B 429 (1998) 369 [hep-ph/9801442] [INSPIRE].

[91] M. Dasgupta and G.P. Salam, Resummation of nonglobal QCD observables, Phys. Lett. B 512 (2001) 323 [hep-ph/0104277] [INSPIRE].

[92] M. Balsiger, T. Becher and D.Y. Shao, Non-global logarithms in jet and isolation cone cross sections, JHEP 08 (2018) 104 [arXiv: 1803.07045] [InSPIRE].

[93] J.M. Campbell, R.K. Ellis, Y. Li and C. Williams, Predictions for diphoton production at the LHC through NNLO in QCD, JHEP 07 (2016) 148 [arXiv:1603.02663] [INSPIRE].

[94] J.M. Campbell, D. Wackeroth and J. Zhou, Study of weak corrections to Drell-Yan, top-quark pair, and dijet production at high energies with MCFM, Phys. Rev. D 94 (2016) 093009 [arXiv: 1608.03356] [INSPIRE].

[95] A. Banfi et al., Jet-vetoed Higgs cross section in gluon fusion at $N^{3} L O+N N L L$ with small-R resummation, JHEP 04 (2016) 049 [arXiv: 1511.02886] [INSPIRE].

[96] A. Buckley et al., LHAPDF6: parton density access in the LHC precision era, Eur. Phys. J. $C 75$ (2015) 132 [arXiv:1412.7420] [inSPIRE].

[97] J. Gaunt, M. Stahlhofen, F.J. Tackmann and J.R. Walsh, $N$-jettiness Subtractions for NNLO QCD Calculations, JHEP 09 (2015) 058 [arXiv: 1505.04794] [INSPIRE].

[98] R. Boughezal, C. Focke, X. Liu and F. Petriello, $W$-boson production in association with a jet at next-to-next-to-leading order in perturbative QCD, Phys. Rev. Lett. 115 (2015) 062002 [arXiv: 1504.02131] [INSPIRE].

[99] ATLAS collaboration, Measurement of the transverse momentum and $\phi_{\eta}^{*}$ distributions of Drell-Yan lepton pairs in proton-proton collisions at $\sqrt{s}=8 \mathrm{TeV}$ with the ATLAS detector, Eur. Phys. J. C 76 (2016) 291 [arXiv: 1512.02192] [InSPIRE].

[100] CMS collaboration, Measurements of differential $Z$ boson production cross sections in proton-proton collisions at $\sqrt{s}=13 \mathrm{TeV}$, JHEP 12 (2019) 061 [arXiv:1909.04133] [INSPIRE].

[101] CMS collaboration, Measurement of the transverse momentum spectra of weak vector bosons produced in proton-proton collisions at $\sqrt{s}=8 \mathrm{TeV}$, JHEP 02 (2017) 096 [arXiv: 1606. 05864] [INSPIRE].

[102] S. Alekhin, J. Blümlein, S. Moch and R. Placakyte, Parton distribution functions, $\alpha_{s}$, and heavy-quark masses for LHC Run II, Phys. Rev. D 96 (2017) 014011 [arXiv:1701.05838] [INSPIRE]. 
[103] S. Dulat et al., New parton distribution functions from a global analysis of quantum chromodynamics, Phys. Rev. D 93 (2016) 033006 [arXiv: 1506.07443] [INSPIRE].

[104] T.-J. Hou et al., New CTEQ global analysis of quantum chromodynamics with high-precision data from the LHC, Phys. Rev. D 103 (2021) 014013 [arXiv:1912.10053] [INSPIRE].

[105] L.A. Harland-Lang, A.D. Martin, P. Motylinski and R.S. Thorne, Parton distributions in the LHC era: MMHT 2014 PDFs, Eur. Phys. J. C 75 (2015) 204 [arXiv:1412.3989] [INSPIRE].

[106] A.D. Martin, W.J. Stirling, R.S. Thorne and G. Watt, Parton distributions for the LHC, Eur. Phys. J. C 63 (2009) 189 [arXiv:0901.0002] [InSPIRE].

[107] NNPDF collaboration, Parton distributions for the LHC Run II, JHEP 04 (2015) 040 [arXiv:1410.8849] [INSPIRE].

[108] NNPDF collaboration, Parton distributions from high-precision collider data, Eur. Phys. J. C 77 (2017) 663 [arXiv:1706.00428] [INSPIRE].

[109] T. Becher and X. Garcia i Tormo, Electroweak Sudakov effects in $W, Z$ and $\gamma$ production at large transverse momentum, Phys. Rev. D 88 (2013) 013009 [arXiv:1305.4202] [INSPIRE].

[110] T. Becher and X. Garcia i Tormo, Addendum: Electroweak Sudakov effects in $W, Z$ and $\gamma$ production at large transverse momentum, Phys. Rev. D92 (2015) 073011 [arXiv: 1509.01961] [INSPIRE].

[111] S. Dittmaier, A. Huss and C. Schwinn, Mixed QCD-electroweak $\mathcal{O}\left(\alpha_{s} \alpha\right)$ corrections to Drell-Yan processes in the resonance region: pole approximation and non-factorizable corrections, Nucl. Phys. B $\mathbf{8 8 5}$ (2014) 318 [arXiv:1403.3216] [INSPIRE].

[112] ATLAS collaboration, Measurement of the transverse momentum distribution of $W$ bosons in pp collisions at $\sqrt{s}=7 \mathrm{TeV}$ with the ATLAS detector, Phys. Rev. D 85 (2012) 012005 [arXiv:1108.6308] [INSPIRE].

[113] CMS collaboration, Measurement and interpretation of differential cross sections for Higgs boson production at $\sqrt{s}=13 \mathrm{TeV}$, Phys. Lett. B 792 (2019) 369 [arXiv:1812.06504] [INSPIRE].

[114] V. Ahrens, T. Becher, M. Neubert and L.L. Yang, Origin of the large perturbative corrections to Higgs production at hadron colliders, Phys. Rev. D 79 (2009) 033013 [arXiv:0808.3008] [INSPIRE].

[115] V. Ahrens, T. Becher, M. Neubert and L.L. Yang, Renormalization-group improved prediction for Higgs production at hadron colliders, Eur. Phys. J. C 62 (2009) 333 [arXiv: 0809.4283] [INSPIRE].

[116] F. Caola, J.M. Lindert, K. Melnikov, P.F. Monni, L. Tancredi and C. Wever, Bottom-quark effects in Higgs production at intermediate transverse momentum, JHEP 09 (2018) 035 [arXiv: 1804.07632] [INSPIRE].

[117] T. Neumann, NLO Higgs+jet production at large transverse momenta including top quark mass effects, J. Phys. Comm. 2 (2018) 095017 [arXiv: 1802. 02981] [InSPIRE].

[118] T. Neumann and C. Williams, The Higgs boson at high p $p_{T}$, Phys. Rev. D 95 (2017) 014004 [arXiv: 1609.00367] [INSPIRE].

[119] L. Budge, J.M. Campbell, G. De Laurentis, R.K. Ellis and S. Seth, The one-loop amplitudes for Higgs +4 partons with full mass effects, JHEP 05 (2020) 079 [arXiv:2002.04018] [INSPIRE]. 
[120] J.M. Campbell, R.K. Ellis and S. Seth, $H+1$ jet production revisited, JHEP 10 (2019) 136 [arXiv: 1906.01020] [INSPIRE].

[121] R. Boughezal, C. Focke, W. Giele, X. Liu and F. Petriello, Higgs boson production in association with a jet at NNLO using jettiness subtraction, Phys. Lett. B 748 (2015) 5 [arXiv: 1505. 03893] [INSPIRE].

[122] S. Catani, L. Cieri, D. de Florian, G. Ferrera and M. Grazzini, Diphoton production at hadron colliders: a fully-differential QCD calculation at NNLO, Phys. Rev. Lett. 108 (2012) 072001 [Erratum ibid. 117 (2016) 089901] [arXiv:1110.2375] [INSPIRE].

[123] L. Cieri, F. Coradeschi and D. de Florian, Diphoton production at hadron colliders: transverse-momentum resummation at next-to-next-to-leading logarithmic accuracy, JHEP 06 (2015) 185 [arXiv:1505.03162] [INSPIRE].

[124] C. Balázs, E.L. Berger, P.M. Nadolsky and C.P. Yuan, All-orders resummation for diphoton production at hadron colliders, Phys. Lett. B 637 (2006) 235 [hep-ph/0603037] [INSPIRE].

[125] C. Balázs, E.L. Berger, P.M. Nadolsky and C.P. Yuan, Calculation of prompt diphoton production cross-sections at Tevatron and LHC energies, Phys. Rev. D 76 (2007) 013009 [arXiv: 0704.0001] [INSPIRE].

[126] P.M. Nadolsky, C. Balázs, E.L. Berger and C.P. Yuan, Gluon-gluon contributions to the production of continuum diphoton pairs at hadron colliders, Phys. Rev. D 76 (2007) 013008 [hep-ph/0702003] [INSPIRE].

[127] Z. Bern, A. De Freitas and L.J. Dixon, Two loop amplitudes for gluon fusion into two photons, JHEP 09 (2001) 037 [hep-ph/0109078] [INSPIRE].

[128] Z. Bern, L.J. Dixon and C. Schmidt, Isolating a light Higgs boson from the diphoton background at the CERN LHC, Phys. Rev. D 66 (2002) 074018 [hep-ph/0206194] [INSPIRE].

[129] S. Catani and B.R. Webber, Infrared safe but infinite: soft gluon divergences inside the physical region, JHEP 10 (1997) 005 [hep-ph/9710333] [INSPIRE].

[130] S. Catani, L. Cieri, D. de Florian, G. Ferrera and M. Grazzini, Diphoton production at the LHC: a QCD study up to NNLO, JHEP 04 (2018) 142 [arXiv: 1802.02095] [INSPIRE].

[131] ATLAS collaboration, Measurement of isolated-photon pair production in pp collisions at $\sqrt{s}=7 \mathrm{TeV}$ with the ATLAS detector, JHEP 01 (2013) 086 [arXiv:1211.1913] [INSPIRE].

[132] ATLAS collaboration, Measurements of integrated and differential cross sections for isolated photon pair production in pp collisions at $\sqrt{s}=8 \mathrm{TeV}$ with the ATLAS detector, Phys. Rev. D 95 (2017) 112005 [arXiv:1704.03839] [InSPIRE].

[133] ATLAS collaboration, Measurement of the $Z\left(\rightarrow \ell^{+} \ell^{-}\right) \gamma$ production cross-section in $p p$ collisions at $\sqrt{s}=13 \mathrm{TeV}$ with the ATLAS detector, JHEP 03 (2020) 054 [arXiv: 1911.04813] [INSPIRE].

[134] M. Grazzini, S. Kallweit, D. Rathlev and A. Torre, $Z \gamma$ production at hadron colliders in NNLO QCD, Phys. Lett. B 731 (2014) 204 [arXiv:1309.7000] [INSPIRE].

[135] M. Grazzini, S. Kallweit and D. Rathlev, $W \gamma$ and $Z \gamma$ production at the LHC in NNLO QCD, JHEP 07 (2015) 085 [arXiv: 1504.01330] [INSPIRE].

[136] D.Y. Shao, C.S. Li and H.T. Li, Resummation prediction on Higgs and vector boson associated production with a jet veto at the LHC, JHEP 02 (2014) 117 [arXiv:1309.5015] [INSPIRE].

[137] Y. Li and X. Liu, High precision predictions for exclusive VH production at the LHC, JHEP 06 (2014) 028 [arXiv:1401.2149] [INSPIRE]. 
[138] S. Alioli, A. Broggio, S. Kallweit, M.A. Lim and L. Rottoli, Higgsstrahlung at NNLL'+NNLO matched to parton showers in GENEVA, Phys. Rev. D 100 (2019) 096016 [arXiv: 1909.02026] [INSPIRE].

[139] S. Dawson, T. Han, W.K. Lai, A.K. Leibovich and I. Lewis, Resummation effects in vector-boson and Higgs associated production, Phys. Rev. D 86 (2012) 074007 [arXiv: 1207.4207] [INSPIRE].

[140] R. Boughezal et al., Z-boson production in association with a jet at next-to-next-to-leading order in perturbative QCD, Phys. Rev. Lett. 116 (2016) 152001 [arXiv:1512.01291] [INSPIRE].

[141] J.M. Campbell, R.K. Ellis and C. Williams, Direct photon production at next-to-next-to-leading order, Phys. Rev. Lett. 118 (2017) 222001 [Erratum ibid. 124 (2020) 259901] [arXiv: 1612.04333] [INSPIRE].

[142] J.M. Campbell, R.K. Ellis and C. Williams, Driving missing data at the LHC: NNLO predictions for the ratio of $\gamma+j$ and $Z+j$, Phys. Rev. D 96 (2017) 014037 [arXiv:1703.10109] [INSPIRE].

[143] M.-x. Luo, T.-Z. Yang, H.X. Zhu and Y.J. Zhu, Quark transverse parton distribution at the next-to-next-to-next-to-leading order, Phys. Rev. Lett. 124 (2020) 092001 [arXiv: 1912.05778] [INSPIRE].

[144] M.A. Ebert, B. Mistlberger and G. Vita, Transverse momentum dependent PDFs at $N^{3} L O$, JHEP 09 (2020) 146 [arXiv:2006.05329] [INSPIRE]. 